Gunter Gebauer, Thomas Alkemeyer,

UWe Flick, Bernhard Boschert,

ROBERT SCHMIDT

\title{
Treue zum Stil
}

Die aufgeführte

Gesellschaft

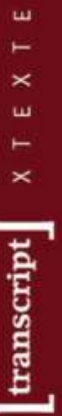

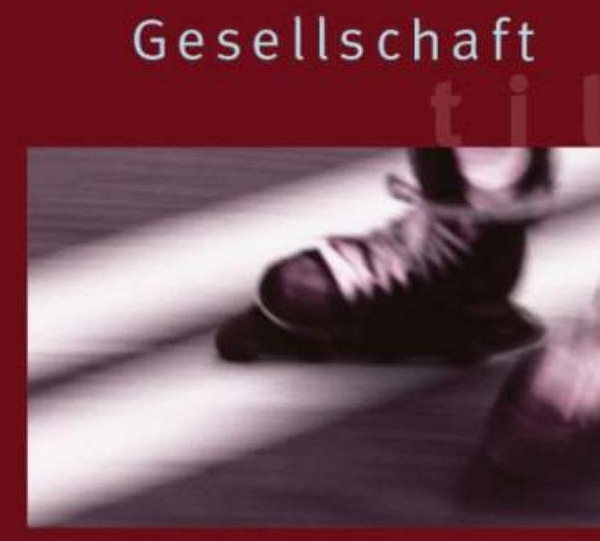


Treue zum Stil 
Gunter Gebauer ist Professor für Philosophie und Sportsoziologie an der FU Berlin, Thomas Alkemeyer ist Professor für Sportsoziologie an der Universität Oldenburg, Uwe Flick ist Professor an der Alice Salomon-Fachhochschule in Berlin, Bernhard Boschert (Dr. phil.) und Robert Schmidt (Dr. phil.) sind wissenschaftliche Mitarbeiter im Sonderforschungsbereich »Kulturen des Performativen« an der FU Berlin. 
Gunter Gebauer, Thomas Alkemeyer, Bernhard Boschert, Uwe Flick, Robert SCHMidT

Treue zum Stil.

Die aufgeführte Gesellschaft

[transcript] $] \times T$ E $\quad X \quad T \quad E$ 


\section{Bibliografische Information der Deutschen Bibliothek}

Die Deutsche Bibliothek verzeichnet diese Publikation in der Deutschen Nationalbibliografie; detaillierte bibliografische Daten sind im Internet über http://dnb.ddb.de abrufbar.

(C) 2004 transcript Verlag, Bielefeld

\section{(c) $\$($ This work is licensed under a Creative Commons BY NC ND Attribution-NonCommercial-NoDerivatives 3.0 License.}

Umschlaggestaltung und Innenlayout: Kordula Röckenhaus, Bielefeld Satz: digitron $\mathrm{GmbH}$, Bielefeld

Druck: Majuskel Medienproduktion GmbH, Wetzlar ISBN 3-89942-205-8

Gedruckt auf alterungsbeständigem Papier mit chlorfrei gebleichtem Zellstoff.

Besuchen Sie uns im Internet: http://www.transcript-verlag.de

Bitte fordern Sie unser Gesamtverzeichnis und andere Broschüren an unter:info@transcript-verlag.de 


\section{Inhalt}

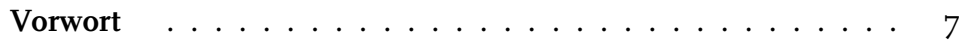

I. Die aufgeführte Gesellschaft $\ldots \ldots \ldots \ldots$. . . . . . 9

Dargestellte Körper . . . . . . . . . . . . . . Iо

Das Spiel als Medium $\ldots \ldots \ldots \ldots \ldots \ldots$ I2

Formen der Vergemeinschaftung $\ldots \ldots \ldots \ldots \ldots$ I4

Die Indikatorfunktion der Spiele $\quad \ldots \ldots \ldots \ldots$. . . . . . . . . . . .

Die Anlage der empirischen Untersuchung $\ldots \ldots \ldots$. . . . . . . . . .

Zur Methodologie . . . . . . . . . . . . . 20

Die Kapitel im Überblick . . . . . . . . . . . . . . . . . . 22

Anmerkungen ................... 23

II. Spiel-Räume $\ldots \ldots \ldots \ldots \ldots \ldots$

Einschließung und Entgrenzung $\ldots \ldots \ldots \ldots . \ldots 27$

Die Orte des Geschehens . . . . . . . . . . . . . 3

Hallenhandball: der geronnene Raum $\quad \ldots \ldots \ldots$. . . . . 32

Triathlon: der überschrittene Raum . . . . . . . . . . . . 34

Inlinehockey: der verflüssigte Raum $\ldots \ldots \ldots \ldots$

Anmerkungen . . . . . . . . . . . . 40

III. Stilgemeinschaften _ . . . . . . . . . . . . 4 45

Hallenhandball: unter dem Dach des Vereins $\quad \ldots . . . . .447$

Triathlon: Gemeinschaft der Individualisten $\ldots . . . . . \quad$ 5I

Inlinehockey: Ad-hoc-Gemeinschaften . . . . . . . . . . 54 
Rahmung versus Attribute . . . . . . . . . . . . . . 57

Die performative Erzeugung der $>$ Vereinsfamilie $<$. . . . . . . . 59

Die Rahmungen der neuen Sportszenen . . . . . . . . . . . . . . . . . . . 66

Anmerkungen ...................66

IV. Gleiten, Rollen, Schweben . . . . . . . . . . . . . . 69

Technik als Gegen-Stand . . . . . . . . . . . . . . . . . . . . . .

Technik als Selbststeigerung .............. 7 I

Technik als Selbsterfahrung . . . . . . . . . . . 74

Technik als Risiko . . . . . . . . . . . . . . . . . 78

Technik als Mimikry . . . . . . . . . . . . . 80

Anmerkungen ................. 8I

V. Das Brodeln im Kleinbürgertum . . . . . . . . . . . . . . 87

Die turbulente Mitte des sozialen Raumes . . . . . . . . . . . . 89

Stetigkeit und Beharrung: Die Handballerin Monika . . . . . 92

Abgebremste und umgelenkte Aufwärtsmobilität:

Der Triathlet Dirk . . . . . . . . . . . . . . . . . . . . . . . . . . . . .

Sog zur Mitte und Besonderung . . . . . . . . . . . . . . . . . . . . . . . . . . .

Zwei konträre Selbstverhältnisse . . . . . . . . . . . . Iо०

Die Veränderung des Habitus aus der Entstehung

eines neuen Selbstverhältnisses . . . . . . . . . . . . I04

Sport als soziale Repräsentationsarbeit . . . . . . . . . . . IIo

Anmerkungen .................. II2

VI. Treue zum Stil . . . . . . . . . . . . . . II7

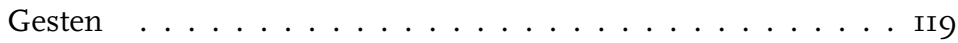

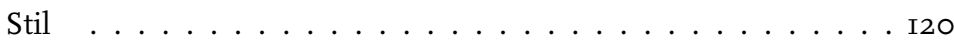

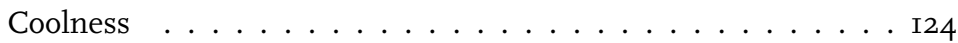

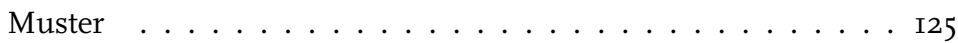

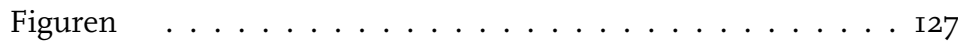

Helden . . . . . . . . . . . . . . . I28

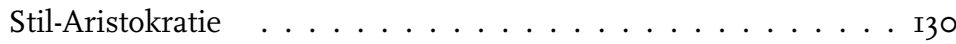

Anmerkungen ...................... I33

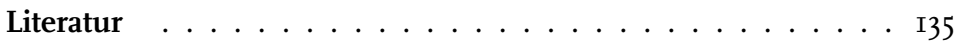




\section{Vorwort}

Mit diesem Band stellen wir Ergebnisse vor, die wir im Rahmen des Sonderforschungsbereichs 447 »Kulturen des Performativen« unter dem Titel »Die Aufführung der Gesellschaft in Spielen« erarbeitet haben. Ein Bericht, der die Anstrengungen und Mühen der Forschung durch umfangreiche Zahlenwerke, Dokumentationen, Interviewtranskripte vor seinem Publikum demonstriert, fordert von diesem eine respektvolle Haltung ein. Wir haben uns mit diesem Band ein anderes Ziel gesetzt, als von unseren Lesern etwas zu verlangen: Wir möchten ihnen alte und neue Spiele im Vergleich zeigen, sie an den Orten des Geschehens herumführen, auf neue Weisen der Vergemeinschaftung und auf einen veränderten Umgang mit Technik aufmerksam machen. Unsere Absicht ist, sie anzuregen, gemeinsam mit uns über die soziologischen und anthropologischen Bedeutungen des Nebeneinanders - oder besser: Gegeneinanders - verschiedenartiger Sportpraktiken der Gegenwart nachzudenken. Wenn sie dabei Vergnügen am Entdecken gewinnen, ist unser Ziel erreicht.

Ganz ohne Hinweise auf den jahrelangen Forschungsprozess kommt auch dieses Vorwort nicht aus. Es waren viele Leute daran beteiligt, oft mit umfangreichen Beiträgen, ohne die unsere Ergebnisse nicht zustande gekommen wären. Die Organisation der Arbeiten lag während der Anlaufzeit in den Händen von Anja Wiedenhöft. An der Erhebung der Daten und ihrer Auswertung waren Vanessa Schwabe und Martin Stern, Eva Vleugels und Silvia Kauric beteiligt. Vanessa Schwabe besorgte auch, zusammen mit Alexandra Backasch, 
die Manuskriptformatierung und übernahm wichtige Korrekturarbeiten. Martin Stern stellte uns seine Feldbeschreibung des Handballs zur Verfügung. Von Norbert Richter erhielten wir die Anregung für die Formulierung des Obertitels unseres Buches.

Von der Deutschen Forschungsgemeinschaft und der Freien Universität wurde unser Vorhaben großzügig gefördert. Gertrud Lehnert und Jens Roselt, in der Geschäftsführung des Sonderforschungsbereichs, und Sabine Lange in dessen Sekretariat haben uns, wenn wir ihre Hilfe brauchten, tatkräftig beigestanden. Unverzichtbar war die Unterstützung, die wir in der ersten Phase von Brigitte Akkoyunlu, in der zweiten von Ina-Maria Gumbel erhalten haben.

Ihnen allen möchten wir herzlich für ihre wichtige Hilfe danken. Unser Dank gilt auch den Interviewpartnern, die uns ihre Zeit und ihr Wissen zur Verfügung gestellt haben. Sie sind alle, unter veränderten Namen, im Text präsent, viele von ihnen mit ihren wörtlichen Aussagen.

Die fünf Autoren des Bandes kommen aus vier verschiedenen Wissenschaftsdisziplinen. Wir haben uns entschlossen, die individuellen Unterschiede nicht in einem buntscheckigen Text abzubilden, sondern über die individuellen Unterschiede hinweg einem gemeinsamen Stil treu zu bleiben.

Berlin, im März 2004

Die Autoren 


\section{Die aufgeführte Gesellschaft}

Wer heute einen realistischen Film über das alltägliche Leben in einer Großstadt dreht, wird vieles im Bild erfassen, was in den Goer Jahren noch nicht zu sehen war. Die typischen Bilder des Stadtraums haben sich verändert; neue Szenerien, Körperpraxen und Verhaltensweisen sind entstanden. Es erscheinen jetzt neue Rollen, gespielt von Personen, die auf andere Weise auftreten, als man es früher gewohnt war. Sie setzen ihre Körper mit neuen Gesten, Ritualen, Bewegungs- und Darstellungsformen in Szene. Der Raum des Sichtbaren, in dem sich die sozialen Akteure zeigen, ist um ein Vielfaches erweitert worden. Im Mittelpunkt dieser Veränderungen steht der Körper mit seinen Praktiken und Darbietungsformen.

In einer Filmproduktion aus den Goer Jahren über die Großstadt hätte der demonstrativ vorgeführte Körper noch keine wichtige Rolle gespielt. Wenn der Körper überhaupt in den Fokus der Sichtbarkeit getreten wäre, hätte man ihn in einem zurückhaltenden, andeutenden Verhalten gezeigt. Er hätte wenig Gelegenheit zur Selbstdarstellung, erst recht nicht zur Show erhalten. Nackte Haut verbargen die Menschen der Stadt schamvoll voreinander; der Körper war kein Objekt von Design. Im früheren Film fehlt gerade das, was das Großstadtleben heute aufregend und bis zum Überdruss >sexy< macht (vgl. Bech I995). Das demonstrative Spiel mit dem Körper, seine auf Wirkung berechnete Formung, seine sportlich-trainierten Bewegungen kamen damals kaum vor: es gab keine vorbeigleitenden Inlineskater, keine vorbeihuschenden Bikes, keine krachenden Skateboards auf den 
Treppenstufen der Parks, keine Jogger, die in exhibitionistischer Sportkleidung ihre Kilometer auf dem Trottoir bolzen, keinen Citymarathon, kein Bungeejumping vom Baukran.

Die frühere Zeit war durch eine Skepsis gegenüber dem Körper geprägt. Das Körperliche wurde weggedrängt und für unwichtig erklärt. Zwar gab es schon den Glamour von Filmschauspielern und Helden des Sports, aber deren körperliche Präsentationsweise und Inszenierung hatten im Kontext vorherrschender Körperzurückhaltung noch den Charakter des Außergewöhnlichen und Exklusiven. In dieser mit bildungsbürgerlichen Idealen festgezurrten, körperdistanzierten Welt taten sich Risse, später auch Brüche auf, aus denen allmählich eine bunte, vitale, hyperaktive, körperverliebte neue Welt emporstieg. Für diesen Aufstieg gibt es viele Gründe; der wichtigste verweist auf die Struktur und Dynamik moderner Gesellschaften selbst. In dieser werden sinnlich dargestellte Distinktionen immer wichtiger. Das Sensorium zur Erfassung von sinnlichen Unterscheidungen ist der soziale Geschmack (vgl. Bourdieu I982). Alle gesellschaftlich Handelnden verfügen über einen sozialen Sinn, der ihnen Geschmacksunterschiede und -gleichheiten meldet. Geschmacksqualitäten werden körperlich ausgedrückt und sinnlich erfasst. In dieser Sichtweise steht der Körper als Produzent von Strukturierungsmerkmalen im Zentrum der Gesellschaft.

\section{Dargestellte Körper}

Wie wurden in den Goer Jahren die Körper dargestellt? Wo wurden spielerische Verhaltensweisen gezeigt? Der Ort des Körperlichen war der Sportverein, der aus dem städtischen Leben ausgegliedert war. Sport wurde als Wettkampf- und Breitensport in einer idealen Welt von genormten Hallen, Stadien, Schwimmbecken und Spielfeldern betrieben. Um zu diesen Orten der Stoppuhren, Bandmaße, Regelbücher und Ergebnislisten Zugang zu erhalten, musste man Mitglied eines Vereins werden oder Eintritt bezahlen. Hier, hinter den Mauern der Sportinstitutionen, waren die Athleten geschützt vor störenden Einflüssen, Unverständnis, Zufällen und Gaffern.

Ebenso wie sich der Sport in seine Spezialräume einschloss und 
in der breiten Öffentlichkeit auf Unverständnis stieß, blieben Haut und Muskeln, Arme und Beine, Oberkörper und Bauch - heute alles Orte der Ausstellung von Jugendlichkeit und Attraktivität - verdeckt von den Stoffen der Anzüge, Kostüme, Hemden und Blusen. Die Anziehungskraft des Körpers wirkte aus dem Verborgenen, in einem Spiel von Verdecken und Zeigen, von Scheu und Voyeurismus. Der Körper wurde mit Zurückhaltung dargeboten, um aus Ungesagtem und Ungezeigtem in der Einbildungskraft des Gegenübers ein inneres Reden hervorzubringen. Die Imagination war eine Voraussetzung für das Entstehen von Intimität.

Heute prägt das Schauspielhafte die sozialen Beziehungen; es lädt zum Sehen ein und fordert zugleich Zurückhaltung bei den Sehenden: Der Zeigende bringt die Aufforderung zum Ausdruck: >Sieh mich an, aber berühre mich nicht<, ebenso wie man einen Schauspieler auf der Bühne eingehend und ohne jede Scheu mit Gutachterblick betrachtet, ihm aber nicht zu nahe treten darf. Die immer offenere Darbietungsweise des Körpers beruht auf einem strengen Berührungsverbot. Aus dem zeigenden körperlichen Spiel selbst entsteht keine Intimität (vgl. Foucault 1976).

In der Stadt von heute sind die alten Grenzen, die herkömmlichen Einteilungen, Unterscheidungen und Strukturen, die das Leben und die Öffentlichkeit in den Städten gegliedert haben, vielfach aufgelöst. Die Körper haben die Mauern der Sportstätten gesprengt. Das Attribut Sport durchdringt die gesellschaftlichen Sphären und verbindet sich mit vielen unterschiedlichen Lebensstilen (vgl. Schmidt 2002). Elemente des Sportlichen sind in die allgemeinen Verhaltensweisen eingegangen; die Trainingsjacke, das T-Shirt, die Streifen von Adidas, der Nike Swoof gehören zu den selbstverständlichen Markierungen des Alltagslebens. Sportliche Gestiken und Praktiken, das Exhibitionistische und Körperverliebte, das den Sport schon immer auszeichnete, hat sich über die Bewohner der Stadt und alle ihre öffentlichen Orte verbreitet. Die Städte haben sich eine neue körperliche Darstellungsfähigkeit gegeben und auf diese Weise ihr Bild grundlegend verändert. Heute schwirren Streetballer, Skateboarder, Inlineskater, BMX-Fahrer durch die öffentlichen Räume und codieren die Landkarte der Stadt neu. Wenn sie Zuschauer brauchen, schaffen sie sich ihre Bühne mitten im sinnlichen Gewebe der alltäglichen Lebensweisen - 
im Zickzackkurs durch belebte Einkaufsstraßen, beim Inlinehockey auf beschaulichen innerstädtischen Plätzen oder beim aggressive skating vor den Orten der Hochkultur, vor Museen, Opernhäusern, Kirchen, Bibliotheken. Manchmal sind sie sich selbst genug und bilden an den öden Orten des Sonntags ihr eigenes Publikum, wie auf den verwaisten Parkplätzen vor den großen Einkaufszentren an der städtischen Peripherie.

\section{Das Spiel als Medium}

Die neuen Bewegungspraktiken zeigen die Veränderung herkömmlicher Ordnungen mit ihren motorischen Charakteristiken an. In der traditionellen Bewegungskultur des Sports ist die Berührung des Bodens, der sichere Stand, der Abdruck, das Abspringen, die Landung, das Zurückkommen auf die Erde ein wesentliches Merkmal - Bodenständigkeit gab dem Sportler Sicherheit. Kennzeichnend für die neue Entwicklung ist, dass man zwischen sich und dem Boden technische Elemente einbaut, Rollen und Kugellager, Räder und Federn, die ein reibungsloses Gleiten ermöglichen und auf diese Weise die gemeinsame Kontaktfläche des eigenen Körpers mit der Erde verändern. Diese Bewegungsformen zeigen an, dass etwas in Fluss geraten ist.

Die traditionelle Motorik im Stadtfilm der Goer Jahre erscheint langsam, zurückhaltend, veraltet. Ihr fehlt der Charme des schnellen Rollens und die Symbiose von Körper und technischem Gerät. Aus der Synthese von Hightech und menschlichen Bewegungen entstehen für die Akteure neue Mobilitätsdimensionen. Im Vergleich zu den Passanten - langsame Wesen auf zwei Füßen, die ihnen Platz machen müssen - bilden sie Hochgeschwindigkeitskörper, die durch die urbanen Zentren rasen. Technizität, Geschwindigkeit, flugähnliche Bewegungen - dies sind die Merkmale, die ein neues Prestige behaupten.

Die sportlichen Attribute, die man heute über die Stadt verstreut findet, verbinden sich mit neuen Körperpraktiken. Diesen fehlt das Sportfachliche, das in Regeln, Punkten, Ergebnissen, Rangplätzen ausgedrückt wird, aber sie besitzen viele Merkmale des Sportlichen, 
die sich auf den Körper beziehen - Fitness, Anstrengung, Mut, Wagnis, Risiko, Ausdauer, Koordination, Muskelkraft.

Wie der Sport gehören alle wichtigen Elemente der neuen Praktiken, auch wenn sie sich von diesem entfernen, in die Welt des Spiels. Sie werden innerhalb des Rahmens (vgl. Goffman I996, Bateson I985) von spielerischen Aufführungen (vgl. Fischer-Lichte/Roselt 200I) hervorgebracht und zum Betrachten angeboten. Dies schließt freilich nicht aus, dass sie in nicht-spielerischen Bereichen weiterverwendet werden, beispielsweise mit dem Ziel, ein sportliches Aussehen zu erwerben, mit dem man auf dem Arbeitsmarkt reüssieren kann. Hier gewinnen Meta-Qualifikationen einer allgemeinen Beschäftigungsfähigkeit immer stärkere Relevanz. Das zur Aufführung von Employability eingesetzte Gesten-, Haltungs- und Ausdrucksrepertoire wird im Rahmen des Spiels und unter dessen Bedingungen erzeugt. So wichtig diese mittelbaren Wirkungen für die Akteure auch sein mögen, sie können nur entstehen, weil die Praktiken als Aufführungen eines Spiels, innerhalb dessen Rahmen, zustande gekommen sind: Die alte Formel, Spiel sei dadurch gekennzeichnet, dass es, wie ästhetische Produktionen, ein Selbstzweck sei, ist deshalb wohl nicht falsch, greift aber zu kurz (vgl. Adamowsky 2000). Denn gerade bei den neu entstandenen Spielen kommt es nicht allein darauf an, dass gespielt wird, sondern dass, indem gespielt wird, eine attraktive Praxis mit eigenen Wirkungen zustande kommt.

Das Spiel ist ein Medium, in dem die aufgeführte Handlung eine Fülle sozialer Effekte und Bedeutungen produziert, und dies mit einer großen allgemeinen Verständlichkeit. Im Spiel entwirft sich das Subjekt in seiner Beziehung zu sich selbst und zu seinen Mitspielern. In diesem Entwurf verbinden sich seine motorischen, darstellerischen und imaginativen Fähigkeiten zu einer ins Spiel projizierten Person. Was das Spielmedium außergewöhnlich attraktiv macht, ist die große körperliche Präsenz der Akteure und die Überzeugungskraft, mit der eine gelungene Aufführung bei den Zuschauern, aber auch beim Subjekt selbst wirkt. Die Bewegungspraktiken der neuen Spiele zeitigen Effekte, die für das Leben in der Großstadt heute von hoher Bedeutung sind: Präsentation der eigenen Person, Verwirklichung eines bestimmten Stils, zwanglose Beteiligung an sozialen Kontexten. Das 
Spiel als körperlich darstellende Praxis ist ein Medium wie Fernsehen, Film, Foto und Video - aber es ist live und leibhaftig; es wird vor anderen und mit anderen verwirklicht. Wie alle übrigen Bildmedien konstruiert es die Gegenstände und Personen, die es abbildet.

\section{Formen der Vergemeinschaftung}

In den neuen Spielen im städtischen Raum zeigen sich Formen der Vergemeinschaftung, die von der Soziologie noch nicht recht bemerkt worden sind. Wenn man das Neue dieser Spielpraxen scharf konturieren will, braucht man es nur in dem Gegenlicht zu betrachten, das vom klassischen Sportverein geworfen wird. In einen Verein tritt man einerseits freiwillig ein, aber anderseits gibt man, indem man Mitglied wird, einen Teil seiner personalen Autonomie auf. Diese Doppeldeutigkeit ist das Merkmal der Vergemeinschaftung nach dem Modell des Vertrags. Wie beim klassischen Gesellschaftsvertrag tritt man einem Verband bei, der größer, umfassender und von höherer Bedeutung ist als der Einzelne. Der Beitretende erkennt mit seiner Unterschrift die Vereinssatzung an, die ihn als Mitglied zu einem bestimmten Verhalten verpflichtet. Er gehört zu denen, die vom Verein etwas erhalten den Raum zum Sporttreiben und Zutritt zur Beteiligung an Wettkämpfen -, aber nur unter den vom Verein gesetzten Bedingungen.

Das Vertragsmodell der bürgerlichen Gesellschaft erklärt eine übergeordnete soziale Entität zu einem höheren Gut - die Familie, das Vermögen, den Staat, die Partei, den Verein -, das von allen Mitgliedern zu schützen und zu mehren ist. Dass der Verein ein höheres Gut für die Mitglieder darstellt, zeigen die Werte, die er setzt und die zu respektieren sind. Er erzeugt eine Art Innenraum, in dem sich die Mitglieder entfalten können, der dem Subjekt neue Handlungsmöglichkeiten gibt und dieses in eine auf bestimmte Werte verpflichtete Gemeinschaft integriert. Für die Mitglieder bildet er eine besondere Welt, in die sie mit ihrer Aufnahme eintreten - insofern gehört die Mitgliedschaft im Verein zum Weltverhältnis der in ihm engagierten Subjekte.

Die neuen Spielpraktiken lösen herkömmliche Ordnungen auf: Für Beobachter aus dem traditionellen Sport wirken sie haltlos, ohne 
erkennbare Regeln und Konventionen, ohne Übungsleiter, Trainingszeiten, geregelten Wettkampfbetrieb. Aber es gibt andere, informelle Organisationsformen; im Inlinehockey beispielsweise gibt es Leitfiguren, die über Listen mit Handynummern verfügen und eine SMS an alle schicken, wenn das schöne Wetter am Nachmittag zu einem Spielchen lockt.

In den neuen Sportarten findet der Novize keinen mit Werten gefüllten Raum vor und keine Instanz, die ihn in eine Gemeinschaft integrieren würde. Was er antrifft, ist ein soziales Gebilde, das sich scheinbar zufällig ergeben hat und das die Beteiligten ständig nach ihren Vorstellungen gestalten. Kommt ein Neuer hinzu oder verlässt einer der Spieler die Partie, ergibt sich daraus wieder ein neues Gebilde. Dessen Gestalt hängt zu einem beträchtlichen Teil davon ab, wie die Beteiligten sich selbst auffassen, wie sie sich als Spieler entwerfen. Auch im traditionellen Sport spielt die persönliche Einstellung, die Haltung der einzelnen Spieler eine gewisse Rolle. Aber was das Spiel ist, was es von den Beteiligten verlangt, was es sein soll, bestimmt das Spiel selbst. So kann Fußball elegant, hart, hölzern oder ideenreich gespielt werden, aber diese Eigenschaften sind nur stilistische Variationen eines Grundmusters, das gegenüber den spezifischen Ausprägungen vorrangig ist.

Bei den neuen Spielen gibt es eine solche Priorität des Spiels gegenüber den Spielern nicht: Was es ist, wie es gespielt wird, sein Charakter, seine Merkmale hängen davon ab, wie sich die Spieler in Bezug auf ihre Mitspieler selbst entwerfen und im Spiel zur Geltung bringen. Das Spiel resultiert aus den sozialen Selbstverhältnissen der Beteiligten. Es ist kein höherrangiges Gut, sondern die handelnden Personen definieren das Spiel aufgrund der Vorstellungen, die sie von sich selbst haben und die sie in diesem umsetzen wollen. Ein solches Geschehen kann nicht mehr mit dem Vertragsmodell beschrieben werden. Es konfrontiert die Spieler nicht mit Werten und Regeln, die sie nicht selber eingeführt hätten. Regelhaftes Verhalten entsteht hier aus den Einstellungen der Spieler, die danach streben, ihre Aktivitäten $\mathrm{zu}$ regulieren und wertvoll zu machen. Ändert sich die Zusammensetzung der Spieler, kann es zu neuen Regularien kommen.

Man erkennt daran, wie sehr der Eintritt eines neuen Beteiligten das Spiel verändern kann. Daher ist seine Aufnahme keine einfache 
Angelegenheit: Die Gemeinschaft hat jedes Mal abzuwägen, ob der Neue zu ihr passt. Er muss den ungeschriebenen Code kennen, die Gesten beherrschen, die stillschweigenden Anforderungen erfüllen. Zwar kann niemand explizit sagen, was er genau zu tun hat; aber jeder Insider weiß, was geht und was nicht. In den spezialisierten Zeitschriften und Videoclips der jeweiligen >Szene< findet man einen Fundus an Symbolen, aus dem die Akteure Modelle eines eigenen Stils entnehmen - ein Reservoir, das aus der Beobachtung der Geschehen auf den öffentlichen Plätzen geschöpft wird.

Im Medium der neuen Sportpraktiken kommt alles auf den Stil der Beteiligten an. Er ist viel wirksamer, als es Satzungen und Vereinsstrukturen jemals sein könnten. Das Selbst- und Weltverhältnis, die Fähigkeiten, Kompetenzen und Charaktereigenschaften der Beteiligten kommen nur insofern im Spiel zur Erscheinung und zur Beurteilung, als sie in Gestalt eines besonderen Stils in das Spiel projiziert werden. Der Stil ist das Element, das die neuen Spiele zusammenhält und ihnen Sinn gibt.

Der Stil der neuen Spielpraxen erzeugt keine Bindungen im üblichen Sinn, keine direkten persönlichen Beziehungen zwischen den handelnden Subjekten. Es kommt auf das Persönliche, das Eigene der Person gar nicht an (vgl. Simmel I980). Die Beziehungen zwischen den Spielern sind abhängig davon, dass diese ihren Stil verwirklichen. Daher ist die Dauer der Gemeinschaft abhängig davon, wie oft, wie lange und wie umfassend der Stil realisiert wird. Zu Fortsetzung und Ausweitung der Beziehungen der Spieler untereinander kommt es nur in solchen Bereichen sozialen Handelns, wo der Stil aktualisiert wird. Dies ist nicht nur in Bereichen der populären Kultur und des Vergnügens der Fall, sondern immer häufiger auch in der Arbeitswelt. $^{\text {I }}$

Entscheidend für die neuen Stilgemeinschaften ist ihre Fundierung in einer gemeinsamen Motorik. Selbstbestimmt werden in unzähligen Akten neue Bewegungen eingeübt; sie werden geformt und behalten, so dass sie in anderen Spielsituationen, Tage oder Wochen später, wieder aktiviert werden können. Die Einarbeitung neuer Bewegungsweisen geschieht mit dem ganzen Körper und ruft langfristige Wirkungen hervor. Ihre motorischen Schemata werden Teil des Körpergedächtnisses; sie lagern sich in den motorischen Fähigkeiten 
des Akteurs ab und werden so zu einer Bereicherung und Veränderung der habituellen Bewegungsweisen der Person. In den neuen Körperpraxen werden bei langer intensiver Übung die hier ausgebildeten Bewegungsschemata zu Bestandteilen des körperlichen Habitus (vgl. Bourdieu I979: 139-202 und Krais/Gebauer 2002).

\section{Die Indikatorfunktion der Spiele}

Im Medium des Spiels, das zu neuen Konstruktionen geradezu einlädt, werden gesellschaftliche Veränderungen in einem frühen Stadium sichtbar, früher als in anderen, weniger beweglichen Handlungsbereichen. Hierin begründet sich das Programm einer Soziologie vom Spiel aus (vgl. Caillois I982, Gebauer/Wulf I998). Spiele sind Gelegenheiten, das Selbst neu einzukleiden und es unter den Bedingungen einer spezifischen ludischen Welt auszuprobieren. Allerdings regiert hier keine Beliebigkeit - unser Selbst gestalten wir nicht unabhängig von seiner Verankerung in der sozialen Welt um. Stile hängen mit der sozialen Position zusammen. Gymnasiasten haben meistens andere Stile als Hauptschüler, Studenten andere als Lehrlinge, höhere Angestellte andere als Arbeiter. An der Projektion von Stilen in die neuen Spielpraktiken lassen sich die sozialen Merkmale erkennen, die mit diesen verbunden sind.

Mit Pierre Bourdieu kann man diese Wechselbeziehungen von Spiel und Sozialwelt als Wirkungen des Habitus beschreiben. Alle Akte des handelnden Subjekts stehen in Beziehung zu dem in seiner Lebensgeschichte ausgeprägten Habitus. Im Spiel sind sie freie Akte, aber nicht frei verfügbar. Der Habitus bildet eine Grundschicht, von der alles soziale Handeln seinen Ausgang nimmt. Wenn sich also im Spiel ein Wandel des Verhaltens anzeigt, so ist dieses Indikator einer Habitusveränderung, die alte feste Verhältnisse lockert und eine größere Oberflächenbewegung erlaubt.

Das Spiel ist ein geschützter Raum, dessen Akte nicht den vollen sozialen Konsequenzen wie das Alltagshandeln ausgesetzt sind. Wenn sich im Medium des Spiels ein neues Selbst- und Weltverhältnis der Person und neue soziale Beziehungen formen, erhält der schon in der Tiefe vorbereitete gesellschaftliche Wandel körperliche Gestalt. Das 
Spiel gewährt einen Schutz nicht nur vor sozialen Konsequenzen, sondern auch vor Reflexion und Beurteilung. Es bildet eine Sphäre der naiven Beteiligung. In diesem geschützten Raum wirkt es auf den aufnahmebereiten Habitus zurück und gestaltet diesen in Teilen um.

Unsere Hypothese ist, dass die in den neuen Spielpraktiken ausgeprägten Veränderungen auch in anderen sozialen Feldern auftauchen. Das Spiel kann als Detektor von neuen sozialen Entwicklungen aufgefasst werden. Im Spiel zeigen sich gesellschaftliche Veränderungen, auf die man nur noch nicht aufmerksam geworden ist. Zwischen den Spielen und anderen sozialen Bereichen gibt es untergründig Verbindungen. Man kann sich diese wie hochempfindliche >Leitungen v vorstellen, die Informationen zwischen verschiedenen sozialen Feldern transportieren. In der Regel sind diese Informationen sehr zahlreich, diffus, vieldeutig und lassen kein klares Bild erkennen. Erst wenn sie auf eine bestimmte Spielpraxis treffen, die ihnen die Möglichkeit zur Projektion gibt, formen sie strukturierte Bilder, die für den Beobachter deutbar werden. Das Konzept solcher intermediären Strukturen, die den Informationsfluss zwischen einzelnen sozialen Feldern und spezifischen Spielen organisieren, ist ein theoretisches Konstrukt (vgl. Alkemeyer/Gebauer 2002). Man kann annehmen, dass mit Hilfe solcher Verbindungen das Spiel zu einem Brennspiegel gemacht wird, der eine Fülle unterschiedlicher flüchtiger Informationen zu einem erkennbaren Bild formt.

\section{Die Anlage der empirischen Untersuchung}

Eingangs haben wir uns auf die Suche nach neuen Entwicklungen der Spielkultur in der Gegenwart gemacht, indem wir zwei imaginäre Großstadtfilme miteinander verglichen haben. Tatsächlich spielt sich dieser Vergleich nicht ausschließlich in der Einbildungskraft ab, sondern kann sich auf höchst reale Erfahrungen in der sozialen Wirklichkeit berufen. Für geübte Beobachter ist es durchaus möglich, aus der erfahrenen Wirklichkeit Aufschlüsse über gesellschaftliche Veränderungen $z u$ erhalten. Freilich werden diese immer an einen eingeschränkten und persönlich gefärbten Erfahrungsausschnitt gebunden sein und daher keine verlässliche Grundlage für Generalisierungen 
bieten können. Nun könnte man sich eine Befragung von älteren Personen zur Beschaffenheit der Lebenswirklichkeit in der Großstadt der Goer Jahre vorstellen. Aber dann hinge die Qualität der Untersuchungsergebnisse von der Verlässlichkeit der Erinnerung der befragten Personen ab - bei einer zeitlichen Distanz von 40 Jahren ein nicht einzuschätzender Faktor. Man braucht aber gar keinen direkten Vergleich zweier Beobachtungszeitpunkte anzustreben, wenn man nämlich die Frage nach den neuen Entwicklungen ausschließlich in der Gegenwart untersucht. In der heutigen Zeit gibt es ein Nebeneinander von Neuem und Altem, das sich für eine Untersuchung neuer Entwicklungen von Spielpraxen anbietet. ${ }^{2}$

Im traditionellen Verein mit seiner Sportpraxis gibt es eine institutionelle Form, ein Regel- und Technikverständnis und ein Angebot an Bewegungen, Wettkämpfen, Auszeichnungen, Weisen der Vergemeinschaftung, die sich in vielen Clubs im Vergleich zu den Goer Jahren nicht wesentlich verändert haben. Wir haben einen Berliner Verein gewählt, der als typisches Beispiel einer gelungenen Tradierung der Einstellungen und des Zusammenhalts der Mitglieder gelten kann, wie es sie mindestens schon seit 40 Jahren gibt. Als besonders geeignet für eine vergleichende Analyse erschien uns die Handballabteilung der Frauen, eine typische Ausprägung eines sowohl auf Wettkampf als auch ein intensives Clubleben orientierten Teils des Vereins.

Die Vergleichsobjekte sind zwei neue Bewegungspraktiken: Triathlon und Inlinehockey. Triathlon ist eine relativ neue Sportart mit wachsenden Teilnehmerzahlen, die in den Medien hohes Interesse findet. Aus drei klassischen Disziplinen zusammengesetzt Schwimmen, Radfahren und Laufen -, ist sie sowohl für ehemalige Vereinssportler auf der Suche nach neuen Bewährungsproben als auch für neue Sportlertypen attraktiv, die sich einem Sportclub sonst nicht anschließen würden. Die Charakteristik der Sportart lässt ein heterogenes Klientel zwischen altem Sportverständnis und neuartiger Suche nach extremen Leistungen erwarten. Einerseits besitzt Triathlon Rahmenelemente des klassischen Sports, wie Vereine und Verbände, andererseits haben die üblichen institutionellen Rahmungen hier keine weitere Bedeutung; es gibt keine Vereinsheime, keine festen Trainingszeiten, keinen engen Zusammenhalt zwischen den 
Mitgliedern. Dafür werden Attribute wie Kleidung und Körperformen sowie die Darstellung der Zugehörigkeit zu dieser Sportart deutlich wichtiger als im klassischen Sport. Als hochgradig individualistische Disziplin verlangt sie ein auf die eigene Person bezogenes Selbst- und Zeitmanagement. Zwar findet man hier Sportgruppen, aber es herrscht eine eher sachliche, unpathetische Auffassung von Gemeinschaft vor, die sich auf die Verfolgung des gleichen Ziels reduziert und darüber hinaus keine Gemeinsamkeiten sucht. Die Gruppe der Triathleten unterscheidet sich durch einen fast exzessiven Körper- und Technikkult von den klassischen Sportgemeinschaften, die von ihnen als konservativ und langweilig beschrieben werden. Eine große Bedeutung haben für sie Askese, Selbstdisziplin und technische sowie diätetische Kenntnisse. In der Wahrnehmung der Athleten ist Triathlon eine exklusive Sportart, die Praxis einer Elite.

Inlinehockey ist von der Institution des Sportvereins noch weiter entfernt als Triathlon. Für die Spieler gibt es keinerlei institutionelle Rahmung. Vielmehr wird die Spielgemeinschaft von Akteuren in eigener Regie immer wieder aufs Neue performativ hergestellt: über Attribute der Mode und der Musik, über Gesten der Begrüßung und des Triumphes, über Rituale des Eintretens und Verlassens des Spielraums, durch die Technologie ihrer Spielgeräte und durch die Setzung von Regeln im Spiel selbst. In dieser neuen Körperpraxis dominiert der Gesichtspunkt des Stils alle Erwägungen, insbesondere die Einschätzung der Regeln.

Vor dem Hintergrund des klassischen Sportspiels Handball lassen sich die beiden neuen Körperpraxen kontrastiv untersuchen; beide können als ausgezeichnete Indikatoren von Veränderungen unserer Gesellschaft Auskunft geben. Als attraktive Medien laden sie zu einer Projektion von Selbstverhältnissen geradezu ein: sie bieten eine Bühne für die Selbstdarstellung der Spieler, ihre Körperpräsentation, ihre Stilistik und ihre Weise, sich als Subjekte zu entwerfen.

\section{Zur Methodologie}

Die skizzierten Veränderungen in Spiel und Gesellschaft lassen sich im Sinne einer theoretischen Zeitdiagnose beobachten. Sie lassen sich 
aber auch anhand konkreter Fälle und Felder des Sporttreibens empirisch untersuchen. Über die Kombination der beiden Zugänge theoretische Zeitdiagnose und empirische Fallanalysen lassen sich verschiedene Perspektiven auf den Wandel des Sports in der Gesellschaft und den vom Sport gezeigten und erzeugten Wandel der Gesellschaft triangulieren. Die hier vorgestellte Untersuchung nimmt eine interdisziplinäre Perspektive ein: Theoretische Analysen werden mit empirischen Materialanalysen kombiniert, in denen der Wandel in den untersuchten Feldern deutlich wird. Dabei werden verschiedene disziplinäre Perspektiven verknüpft. Empirische Zugänge mit Methoden der soziologischen und ethnografischen Forschung werden eingebettet in theoretische Analysen mit den Begrifflichkeiten der Philosophie, Soziologie und Kulturwissenschaften.

Auf der Ebene der empirischen Analysen wird ein aktueller mit einem eher rückblickenden Zugang kombiniert: Neben der teilnehmenden Beobachtung aktueller Sportpraxis in den Feldern Handball, Triathlon und Inlinehockey an jeweils signifikanten Orten werden die Erfahrungen und Bewertungen ausgewählter Teilnehmer und der darin repräsentierte Wandel der Felder in Interviews mit Erzählungen und Fragen rekonstruiert. Darüber hinaus wird die Innenperspektive der Teilnehmer über Befragungen und die Außenperspektive auf die untersuchten Felder durch teilnehmende Beobachtung ermittelt. Leitendes Prinzip der Analyse ist dabei der kontrastierende Vergleich auf verschiedenen Ebenen.

Die einzelnen Fälle werden anhand ihrer Erzählungen und Einschätzungen kontrastierend analysiert. Die in den Interviews erhaltenen Berichte über das Feld und das (eigene oder fremde) Handeln werden mit dem verglichen, was sich in den teilnehmenden Beobachtungen aus einer ethnografischen Perspektive zu Interaktionen, Handlungsroutinen und Markierungen des sozialen Raumes zeigt. Die drei Felder Handball, Triathlon und Inlinehockey als Beispiele unterschiedlich formalisierter Handlungsfelder im Sport werden einer kontrastiven Analyse unterzogen, um die Unterschiede, aber auch die vorhandenen Gemeinsamkeiten zu verdeutlichen. Schließlich werden die induktiv aus diesen Materialanalysen gewonnenen Erkenntnisse mit den deduktiv gewonnenen Erkenntnissen aus den theoretischen Zeitdiagnosen kontrastiert. ${ }^{3}$ 


\section{Die Kapitel IM ÜBerbLick}

Ein zentrales Unterscheidungskriterium zwischen neuen Spielen und traditionellem Vereinssport liegt im je spezifischen Verhältnis zum Raum, das wir zunächst ausleuchten wollen (II). Neben den mobilen subjektivierten Räumen der Inlineskater oder Mountain-Biker existieren in den neuen Spielen auch immobile, personalisierte öffentliche Orte, zu denen die Akteure immer wieder zurückkehren. Beide Raumtypen unterscheiden sich von den Funktionsräumen des traditionellen Sports dadurch, dass sie nur wenig begrenzt und allen zugänglich sind.

In diesen unterschiedlichen sozialen Räumen artikulieren sich auch differente Gemeinschafts- und Selbstverhältnisse. Ihre Analyse steht im Mittelpunkt des folgenden Kapitels (III). Im klassischen Sportverein werden Gemeinschaften innerhalb dauerhafter institutioneller Rahmungen gebildet. In den neuen Spielen werden solche formalen Stützen zugunsten von Offenheit, Unverbindlichkeit und Flüchtigkeit aufgegeben. Hier prägen sich neue Konstellationen von Person, Selbst und Gemeinschaft aus, denen gerade in der »Gesellschaft der Individuen« (Elias I994) besondere Bedeutung zukommt.

Der für die neuen Spiele typische Verzicht auf Sicherheit kommt nicht zuletzt in ihren spezifischen Bewegungsformen des Rollens, Gleitens und Schwebens zum Ausdruck. Diese wiederum werden erst durch neue technische Geräte möglich, die in ein besonderes Verhältnis zum Körper treten, das wir in einem weiteren Kapitel (IV) herausarbeiten.

Die Entwicklungen im Bereich der neuen Spiele vollzieht sich im Kontext sozialen Wandels. Sie verleihen gesellschaftlichen Veränderungen mitunter erst Prägnanz, Gestalt und Sichtbarkeit. Dieser Zusammenhang steht im Mittelpunkt des 5. Kapitels. Über die Rekonstruktion der sozialräumlichen Reisewege ausgewählter Akteure wird hier versucht, wesentliche Signaturen der neuen Spiele aus der sozialräumlichen Dynamik verständlich zu machen.

Das letzte Kapitel (VI) schließlich zeigt, dass die in den neuen Spielen zu beobachtenden Informalisierungs- und Individualisierungsprozesse keineswegs in sozialer Desintegration münden. In ihrer Treue zum Stil bilden die Akteure vielmehr exklusive und elitäre Ge- 
meinden. Hier schließen sich Selbstbezüglichkeit und gemeinschaftliche Bindungen nicht gegenseitig aus; sie steigern sich vielmehr aneinander.

\section{Anmerkungen}

I Es gibt neue Tätigkeitsbereiche jenseits der klassischen Berufsfelder, die ihre Innovation dadurch sichtbar machen, dass sie spielerische Formen in sich aufnehmen (vgl. Meschnig/Stuhr 2003 und Schönberger/Springer 2003).

2 Unsere empirische Untersuchung umfasste drei sorgfältig ausgewählte Sportgruppen (Handball, Triathlon, Inlinehockey), die wir teilnehmend beobachtet haben. Die Feldarbeit begann im Frühjahr I999 und endete im Sommer 200I. Neben der ethnografischen Datenerhebung (Beobachtungsprotokolle, Spontaninterviews) wurden insgesamt 30 episodische Interviews (je Io in jedem Untersuchungsfeld) geführt.

3 Für detailliertere Darstellungen der verwendeten, aber hier nur knapp skizzierten empirischen Zugänge (episodisches Interview, teilnehmende Beobachtung, Fallkontrastierung, Triangulation und Dokumentation der Sozialdaten) vgl. Flick (2002). 



\section{Spiel-Räume}

Wenn man die neuen Spiele mit dem traditionellen, vereinsförmig organisierten Sport vergleicht, springen nicht nur die ganz anderen Bewegungsformen, Sportgeräte und sozialen Organisierungsweisen ins Auge. Es wird zugleich deutlich, dass sich die Unterschiede zwischen alten und neuen Spielen vor allem auch in einer räumlichen Dimension manifestieren: Man spielt heute nicht nur anders als früher, sondern auch in anderen Räumen, auf anderen Plätzen und an anderen Orten. ${ }^{\mathrm{I}}$

Während der institutionalisierte Breiten- und Wettkampfsport sich durch die Geste des Zurückziehens und Einschließens in funktional gestaltete und gegenüber dem Alltagsleben abgeschirmte Sonder-Räume wie Hallen, Stadien und Schwimmbäder auszeichnet, machen die neuen Spiele durch eine Geste des Heraustretens und Ausbrechens auf sich aufmerksam. Ihre Protagonisten sprengen die Grenzen und Begrenzungen des klassischen Sports, kehren seinen Hallen und Stadien den Rücken, um in die öffentlichen Räume und Ballungszentren der Städte auszuschwärmen (vgl. Bette I999) und diese als Repräsentationsforen und Präsentationsbühnen ihrer Selbstdarstellungen und Lebensstile zu nutzen. ${ }^{2}$

Inlineskater, BMX-Radfahrer, Skateboarder, Streetballer oder Inlinehockey-Spieler lassen sich entsprechend nicht nur von den Bewegungsmöglichkeiten ihrer neuen Sportgeräte, sondern auch von der Atmosphäre, der Vielfältigkeit und Lebendigkeit, den Freiheitsgraden und Erfahrungsmöglichkeiten des öffentlichen Raums faszinieren. 
Allerdings: die Räume, in denen sie zusammentreffen und ihre Bewegungskünste aufführen, Fußgängerzonen, Marktplätze, städtische Zentren, öffentliche Repräsentationsbauten wie Kirchen, Bibliotheken, Theaterhäuser oder Museen, Asphaltflächen verlassener Industrieanlagen, U-Bahnschächte und Autobahnbrücken sind keine originären Sporträume. Sie dienen bereits anderen Zwecken und gehorchen anderen Funktionen, entfalten deshalb eigene Diskurse, Mythologien und materiale Widerständigkeiten. Sie müssen entsprechend von den Akteuren der neuen Spiele in einem Prozess sekundärer Rahmung (Goffman I996: 52ff.) erst erobert, als Spiel-Räume markiert, definiert und gestaltet werden. Dabei wird an vorhandene Codierungen und Strukturierungen der Räume angeschlossen, wie diese umgekehrt durch Hinzufügen von neuen Elementen modifiziert werden. Das Entscheidende dabei ist, dass dies in einem besonderen Medium geschieht: dem der körperlichen Aufführung und theatralen Inszenierung von Bewegungen. In diesem körperlichen Ausdruck des Machens und Herstellens, in der Schaffung eines Bedeutungsrahmens, in der sinnlich erfahrbaren Verräumlichung und Verkörperlichung von Bildern, Lebensstilen und Vorstellungswelten, liegt denn auch die besondere performative Qualität (vgl. Fischer-Lichte 200I) der neuen Spiele, ihrer kulturellen Aufführungen (vgl. Singer I959, Alkemeyer 2003) und Inszenierungsformen (vgl. Willems/Jurga I998).

Während dem Raum im Zusammenhang der konventionellen Spiele im Allgemeinen wenig Aufmerksamkeit geschenkt wird und dieser in der Selbstverständlichkeit des Gewohnten verschwindet, allenfalls instrumentell, als technisches, architektonisches, finanzoder repräsentationspolitisches Problem von sich reden macht ${ }^{3}$, weisen die neuen Spiele explizit auf die Kategorie des Raumes hin und stehen damit im Kontext jener gesellschaftlichen Transformationen $^{4}$, die in der jüngeren Vergangenheit zu einer Krise der Raumwahrnehmung und in dessen Folge nicht zuletzt zu einer wissenschaftlichen Renaissance der Raumthematik geführt haben (vgl. Löw 200I und Maresch/Werber 2002). Die neuen Spiele zeigen paradigmatisch die gesellschaftlichen Veränderungen in Bezug auf die Wahrnehmung des Raumes und die Erzeugung von Räumen an.

Das heißt allerdings nicht, dass der Raum in den konventionellen Spielen des Sports eine zu vernachlässigende Größe darstellen würde. ${ }^{5}$ 
Vielmehr verfügen sie mit ihrer unhintergehbaren Körperlichkeit und konstitutiven Bewegungsdynamik immer schon über eine räumliche Dimension (vgl. Boschert 2002). Sie brauchen einerseits den Raum, wie sie durch diesen limitiert sind. Und sie erzeugen andererseits spezifische Spiel- und Funktions-Räume, wie in diesen bestimmte Raumvorstellungen und -wahrnehmungen manifest werden. Sie lassen das Soziale so als besondere Raumerfahrung spürbar werden und sie geben dem Sozialen zugleich selbst eine räumliche Struktur und sinnliche Gestalt. Indem in den Spielen Kämpfe um soziale Anerkennung zur Geltung kommen, Distinktionsprofite verteilt und kulturelle Differenzen aktualisiert werden, beziehen sie sich zugleich auf den sozialen Raum (vgl. Bourdieu I985), korrespondieren mit gesellschaftlichen Reisewegen, Macht- und Ungleichheitsverhältnissen (vgl. Vester et al. 200I).

Die Raumthematik ist also schon für die Entstehungsgeschichte des klassischen Sports zentral. Sie konturiert sich im Laufe der Geschichte in je spezifischer Form und wird dadurch auch unterschiedlich wahrgenommen (vgl. Noller 2000). Indem die neuen Spiele eindringlich auf die Raumdimension aufmerksam machen, zeigen sie gesellschaftliche Irritationen an, die sowohl die Raumwahrnehmung als auch den Begriff des Raumes selbst betreffen.

\section{Einschliessung Und EntgrenzUng}

Das Feld des modernen Sports als ein System von Institutionen und Akteuren (vgl. Bourdieu I992 und I993 sowie Schmidt 2002) ist eine Erfindung der jüngeren Geschichte. Als soziales Handlungsfeld mit eigenständiger Kommunikations- und Feldlogik hat sich der moderne Sport historisch seit der Mitte des I8. Jahrhunderts und beschleunigt vor allem mit der Industrialisierung des I9. Jahrhunderts ausgebildet (vgl. Elias/Dunning 2003). Die Entstehungsgeschichte des modernen Sports lässt sich insofern räumlich beschreiben, als sich ein eigenständiger, sich selbst organisierender, relativ autonomer sozialer Raum des Sports innerhalb des Gesamtraumes des Gesellschaftlichen herausbildet und gegenüber seiner übrigen innergesellschaftlichen Umwelt durch eigene operative Kommunikationsformen abschließt. ${ }^{6}$ 
Neben dieser Ausdifferenzierung des Raumes des Sports lässt sich innerhalb dieses Sonderraumes als ein weiterer Verräumlichungsprozess wiederum die Ausbildung der einzelnen Sportarten beobachten. Dieser ist vor allem durch eine Entwicklung raum-zeitlicher Begrenzungen der jeweiligen sportlichen Bewegungspraxen gekennzeichnet. Wie Elias (1983) und Elias/Dunning (2003) am Beispiel der Geschichte des Fußballs deutlich machen, handelt es sich bei diesem binnenräumlichen Differenzierungsprozess um einen Prozess der Einschließung, Verregelung, Institutionalisierung und Kodifizierung der entstehenden sportlichen Praxisformen einerseits und der räumlichen Trennung von sportlichen Akteuren und Zuschauern andererseits (vgl. Messing/Lames I996). Er mündet schließlich in die Architektonik spezifischer Funktionsräume mit räumlich voneinander getrennten Zuschauerrängen und Spielfeldern ein.

Während die volkstümlichen Vorformen des Sports noch keinen sozialen Sonderraum bilden, sondern in das Alltagsleben integriert sind und noch weitgehend ohne räumliche und zeitliche Begrenzungen auskommen, hat der geregelte und institutionalisierte Wettkampfsport seine eigenen handlungsspezifischen Funktionsräume und raum-zeitlichen Rahmen ausgebildet. ${ }^{7}$ Seine Hallen und Stadien lösen Körperbewegungen aus ihren alltäglichen Funktionszusammenhängen heraus, um sie zu eigenständigen, selbstzweckhaften sportiven Bewegungsformen zu stilisieren (vgl. Bourdieu I992). Diese werden, von Spezialisten ausgeführt, wiederum in einem geregelten konkurrenziellen Kampf um Sieg und Niederlage den Erwartungen eines hoch emotionalisierten Publikums ausgesetzt. Der klassische Wettkampfsport trennt Akteure und Zuschauer scharf voneinander und artikuliert damit zugleich ein neues Verständnis des Öffentlichen: traditionale Formen der repräsentativen Öffentlichkeit werden vom Modell einer bürgerlichen Öffentlichkeit (vgl. Habermas I990) abgelöst. In den neuen Spielen zeigt sich erneut ein Wandel des Verständnisses von Öffentlichkeit, die heute weniger der politischen als vielmehr der kulturellen Repräsentation dient (vgl. Kaschuba 2003).

Mit der skizzierten Entwicklungsgeschichte des modernen Sports, der Isolierung und Perfektionierung von Körperbewegungen in dafür eigens eingerichteten Funktionsräumen ist zugleich der allmähliche Niedergang des urbanen Straßenspiels im Zuge der ökonomischen 
Nutzung des Straßennetzes verbunden. Die Ausdifferenzierung des modernen Sports folgt damit einer allgemeinen zivilisatorischen Entwicklung, die man als »Verhäuslichung « beschreiben kann (Zinnecker I990: I42). Durch die Institutionalisierung separater und geschlossener Handlungsräume werden soziale Handlungen mit Hilfe dauerhafter Befestigungen voneinander isoliert, stabilisiert und berechenbar gemacht. Dieser Zusammenhang von Raumgestaltung, Kontrolle und Produktivitätssteigerung qualifiziert die Moderne als »die Epoche des Raumes« (Foucault I998: 34).

Musterbeispiele sportlicher Funktionsräume sind die nüchternen, hoch funktionalen, DIN-genormten Hallen und Fußballarenen. Meist erst wenige Stunden vor Spielbeginn geöffnet, um nach dem Spiel gleich wieder geschlossen zu werden, bilden Letztere dazwischen einen leeren, ungenutzten Raum in der Dichte und Enge der Stadt, in dem sich nichts ereignet. ${ }^{8}$ Die Bauformen und räumlichen Gliederungen der Sporthallen erlauben es, Körperbewegungen sportartspezifisch zu kodifizieren und disziplinatorisch auszurichten. Die sinnliche Fülle des öffentlichen Lebens wird in diesen Räumen ebenso ausgesperrt wie andere mögliche Störgrößen. Das Ziel solcher räumlichen Strukturierungsformen ist die Fokussierung der Aufmerksamkeit auf die Bewegung und die Leistungsoptimierung. Entsprechend sind es vorrangig leistungssportliche Erfordernisse, die die Form und Gestaltung dieser künstlichen Sporträume bestimmen, etwa die Schaffung eines glatten Untergrundes, die Ausschaltung von Windeinflüssen oder die Unabhängigkeit von natürlichem Tageslicht. In den sportspezifischen Funktionsräumen werden nicht nur Leistungen verglichen, es dokumentiert sich in ihnen zugleich eine bestimmte Sportauffassung und ein bestimmtes Konzept des Umgangs mit dem Körper: einem Körper nämlich, der sich formen, kontrollieren und mit wissenschaftlichen Methoden trainieren und verbessern lässt. In diesem Sinne sind die Räume des organisierten Sports Orte eines Spiels der »gezähmten Körper« (Bale i997).

Im Verräumlichungsprozess des Sports bleibt freilich auch der Raumbegriff nicht konstant; es entfalten sich neue Facetten und Bedeutungsebenen: In der Entstehung transnationaler Sport-Räume (vgl. Pries I996, Bröskamp I998), die sich im Kontext von Globalisierungs-, Universalisierungs- und Internationalisierungsbewegungen in Wirt- 
schaft, Wissenschaft, Recht (vgl. Stichweh 2000), Mode, Popmusik, Kunst oder Architektur vollziehen, wird dies besonders sinnfällig. Entscheidend ist dabei, dass die transnationalen Räume des Sports nationalstaatliche Grenzen überschreiten und ein territoriales Raumverständnis veraltet erscheinen lassen. Es entstehen jedoch nicht nur andere Räume, sondern in diesen kommt zugleich ein neues Raumverständnis zum Ausdruck. In Bezug auf die Prominenz des Räumlichen in den neuen Spielen führt dieser Zusammenhang auf die Frage, welche Raumvorstellungen hier artikuliert und verkörpert werden und welche gesellschaftlichen Veränderungen sich darin ankündigen.

Zeichnet sich die erste Differenzierungsphase des Sports durch die Entmischung und Einschließung sportlicher Praxen aus, so kehrt eine zweite Entwicklung diese Institutionalisierung und Standardisierung wieder um. Indem die Akteure der neuen Spiel- und Bewegungsformen die klassischen Funktionsräume des Sports verlassen und sich den öffentlichen städtischen Räumen zuwenden, an deren materiellen Beschaffenheiten, Möglichkeiten, symbolischen und emotionalen Qualitäten sie spielerisch teilhaben wollen, heben sie die Isolierung, Normierung und Kontrolle der Bewegungen des Körpers in >verhäuslichten s sportlichen Provinzen wieder auf. Sie wenden sich gegen ein bestimmtes Sportverständnis und bringen dies auch dadurch zum Ausdruck, dass sie sich an bestimmten Orten aufhalten. Diese Entwicklung löst den Sonderraum des Sports jedoch keineswegs in einen anomischen Zustand auf. Die neuen Spiele suspendieren vielmehr lediglich die klassischen binnenräumlichen Differenzierungsstrukturen. Zwar werden die neuen Spiele außerhalb der traditionellen Sportanlagen in den Räumen der städtischen Öffentlichkeit gespielt, ${ }^{9}$ dies geschieht aber gerade auf der Grundlage und unter der historischen Voraussetzung eines bereits formierten gesellschaftlichen Feldes des Sports, auf dessen Sportverständnis dabei zugleich Bezug genommen wird. Insofern kann dies auch nicht als ein Zurück - etwa zu Formen des volkstümlichen vormodernen Sports - verstanden werden. Vielmehr ist das Funktionssystem des modernen Sports gerade die Bedingung der Möglichkeit dieser fortschreitenden Ausdifferenzierung der Sportlandschaft. ${ }^{\text {Io }}$ 


\section{Die Orte des Geschehens}

Der öffentliche Platz im alten westlichen Zentrum Berlins, den wir aufgrund seiner kontrastierenden Aussagekraft für unsere Untersuchung ausgewählt haben, ist ein exemplarischer urbaner Sportraum. Er grenzt sich mit seiner zentralen Lage deutlich von den Räumen klassischer Sportstätten ab, die nicht selten am Stadtrand gelegen sind. Die Einbettung des Sporttreibens in das städtische Leben spielt hier eine zentrale Rolle: signifikante Merkmale des Sich-Bewegens und Spielens in der urbanen Öffentlichkeit, der Bühnen- und Aufführungscharakter des Platzes sind hier besonders augenfällig.

Kontrastierend dazu haben wir mit einer Damen-Handballmannschaft in einem nördlichen Wohnbezirk Berlins ein typisches Beispiel des Vereinssports ausgewählt. Anders als bei den informellen Gruppen der neuen Spiele findet der Betrieb des Handballsports in einer dafür vorgesehenen Sporthalle statt. An den inneren und äußeren Spielfeldbegrenzungen oder an den alters-, geschlechts- und leistungsbezogenen Zuordnungen und Segmentierungen zeigt sich, wie sehr die Strukturen und Ordnungsschemata des Vereinssports räumlich materialisiert und verankert sind.

Triathlon, unser drittes Untersuchungsfeld, nimmt unter der Perspektive der Raumthematik zwischen dem vereinsförmig betriebenen, traditionellen Sport und den neuen Spielen eine Zwischenstellung ein. Im Triathlon werden mit dem Radfahren, dem Schwimmen und Laufen drei Sportarten miteinander verbunden, die ansonsten getrennt voneinander ausgeübt werden und organisiert sind. Aus diesem Grund stellt er buchstäblich weitreichende und heterogene Anforderungen an Räume, die sowohl die Schwimmhalle als auch Straßen und Grünflächen einschließen. Triathleten haben eine spezielle Art des Umgangs mit dem Raum: Dieser wird nicht nur angeeignet, sondern regelrecht verbraucht und konsumiert.

Die hier kurz skizzierten drei Untersuchungsfelder sollen im Folgenden näher vorgestellt und in Hinsicht auf die unterschiedlichen Räume, die sie jeweils aufspannen, miteinander verglichen und theoretisch ausgewertet werden. 


\section{Hallenhandball: DER geronnene Raum}

Das Feld Handball weist vielfältige und zugleich in hohem Maße ortskonkrete Räume auf, in denen sich die Handballerinnen in klar definierten und verbindlichen raum-zeitlichen Strukturen bewegen. Der von uns untersuchte typische Großverein verfügt über ein eigenes Vereinsgebäude und dem Angebot der Sportarten entsprechend über eine differenzierte räumliche Ausstattung: zwei Sporthallen, Sportplatz, Tennisplätze, Tanzraum, Kraftraum, Kegelbahnen. Handball bildet dabei neben mehreren anderen Sportarten eine Abteilung des Großvereins.

Im Eingangsbereich des Vereinsgebäudes, der unter anderem für Informationsregale und diverse Schaukästen genutzt wird, werden über Plakate und Aushänge auch die Handballabteilung betreffende Sitzungen, Festivitäten, bevorstehende Wettkämpfe u.Ä. angekündigt und Einladungen an prinzipiell jeden Interessenten ausgesprochen. Die von uns beobachtete Damen-Handballmannschaft kommt zweimal wöchentlich zum Training zusammen, davon einmal in der vereinseigenen Sporthalle, das zweite Mal in einer anderen Halle im selben Bezirk. Die vorwiegend am Wochenende stattfindenden Wettkampfspiele werden in den über die gesamte Stadt verteilten Vereinshallen ausgetragen. Die Räume, in denen das Spiel betrieben wird, sind architektonisch spezifisch gestaltet und als solche eigens für den Sport geschaffen. Neben einer klaren Trennung nach außen weisen sie im Inneren eine durch Spielfeldgrenzen, Markierungen und Tore standardisierte und auf die Bedürfnisse der sportlichen Praxis zugeschnittene Strukturierung auf. Räumliche Strukturen sind aber auch außerhalb des Spielfeldes, z.B. hinsichtlich der Geschlechtertrennung in Form separierter Umkleidekabinen und Sanitäranlagen, zu beobachten. Des Weiteren sind der Geräteraum und der Zuschauerbereich vom Raum der sportlichen Handlungspraxis abgegrenzt.

Die Ausübung des Handballspiels ist auf spezifische und hochgradig normierte sportliche Sonder-Räume angewiesen. Die Möglichkeit verbindlicher Nutzungsrechte von Hallen zählt deshalb zu den strukturellen Bedingungen des Feldes. Die raum-zeitliche Rahmung ist dabei Grenzziehung und Ausgrenzung zugleich. Sie schafft für die Akteure einen Innenraum, der sie zusammenschweißt und ihnen 
zugleich das Gefühl von Exklusivität durch Abgrenzung nach außen verschafft.

Anders als die urbanen Räume der neuen Spiele wird der Raum des Handballs nicht erst durch das sportliche Geschehen selbst erzeugt; der Raum gibt der sportlichen Praxis als gebaute Institution vielmehr eine klare Struktur und Führung. Anders als bei den neuen Spielen existieren diese Räume auch nicht nur für den Augenblick. Sie emergieren nicht erst als Moment von körperlichen Aufführungen. Sie sind vielmehr dauerhaft und existieren auch dann noch weiter, wenn sie verlassen werden und die sportliche Praxis beendet ist. Anders als in den neuen Spielen, deren turbulente und temporäre Räume ein hohes Maß an stilsicherem Orientierungswissen voraussetzen, kann der Sportraum im Handball wie eine Handlungsanleitung gelesen werden.

Festgelegte Trainings- und Wettkampfzeiten geben dem Alltagsleben der Mannschaftsmitglieder eine rhythmische Struktur. Die raum-zeitlichen Bedingungen der Sportpraxis bilden somit neben einem festgeschriebenen Reglement und der Segmentierung der Vereinsmitglieder in geschlechts-, leistungs- sowie altershomogene Mannschaften eine strukturelle Rahmung, die wesentlich die soziale Kohäsion im Verein erzeugt und wie ein Stützkorsett dem Körper der Gemeinschaft Form und Halt gibt. ${ }^{\text {II }}$

Auch in der Sportpraxis selbst wird soziale Zugehörigkeit räumlich greifbar: Das Hallenhandballspiel zentriert sich auf die beiden Torräume. Das übrige Spielfeld wird im klaren Nacheinander von verteidigender und angreifender Mannschaft überbrückt. Vor dem Wurfkreis, der farblich hervorgehoben ist, formiert sich die verteidigende Mannschaft als eine geschlossene Abwehrkette. Die angreifende Mannschaft tritt als geschlossene Angriffskette auf. Effekte räumlicher Plastizität ergeben sich in der Wettkampfpraxis zudem durch die einheitliche Wettkampfbekleidung, die in Farbe und Schnitt gleichen Trikots sowie in der Aufwärmphase durch die einheitlichen Trainingsanzüge. Die unterschiedliche Kleidung der beiden Mannschaften macht für den außenstehenden Betrachter überhaupt erst deutlich, dass die beiden Mannschaften jeweils unterschiedliche Territorien verteidigen. Die territoriale Aufteilung des Spielfeldes vollzieht sich noch einmal in einer Zweiteilung des Zuschauerraums. Die Angehö- 
rigen, Freunde und Familienmitglieder der Mannschaften gruppieren sich auf jeweils einer Seite der Tribüne.

\section{Triathlon: Der überschrittene Raum}

Im Triathlon muss man - im Unterschied zu vielen konventionellen Vereinssportarten, die sich auf einen architektonischen Raum wie die Turnhalle oder das Stadion konzentrieren - im Plural von den Räumen dieser Sportart sprechen. Die verschiedenen Räume des Triathlons machen dessen Zwitterstellung zwischen traditionellem und postkonventionellem Sport besonders deutlich. Sie reichen von den Schwimmhallen, wo - oft zusammen mit Mitgliedern von Schwimmvereinen und unter deren organisatorischer Regie - zu festen Zeiten das Schwimmtraining stattfindet, über die zumeist individuell oder in kleinen Grüppchen absolvierten und immer wieder neu festgelegten Fahrrad- und Laufstrecken an den Stadträndern und Grünflächen bis hin zu den besonderen Orten, an denen dann die Zusammenkünfte und Wettkämpfe der Triathleten stattfinden. Dabei sind nur die Schwimmhallen und die Orte der Wettkämpfe einer ethnografischen Beobachtung zugänglich: Nur hier versammeln sich die Triathleten, nur hier konstituiert sich also die Kultur der zu beschreibenden >Ethnie< (vgl. Reichertz I992) wirklich ortskonkret, nur hier hat das Feld Triathlon klare physische Raumgrenzen.

Triathlon bedeutet im Vergleich zum herkömmlichen Sport nicht nur eine leistungsmäßige und zeitliche, sondern auch eine räumliche Ausweitung. Die sportliche Praxis beginnt in der Schwimmhalle, an einem klar abgegrenzten Ort, um diese räumlichen Grenzen jedoch aufzusprengen und zu überschreiten. Die Praxis des Triathlons bildet mit ihrer Struktur die Veränderungen zwischen begrenztem, an spezielle Orte und feste Zeiten gebundenen Sport und postkonventionellen Sportpraxen ab, die aus diesen Grenzen ausbrechen und bislang vom Sport unberührte Räume als Handlungsfelder reklamieren.

Für die Triathletin Susanne ist das Schwimmtraining »der Fixpunkt, wo man sich immer sieht«. In der Schwimmhalle treffen sich die Triathleten $\mathrm{zu}$ festen Trainingszeiten, hier sind sie meistens mit 
anderen Vereinsschwimmern und - während des öffentlichen Badebetriebes - mit Freizeitschwimmern räumlich zusammengefasst. Um möglichen Verwechslungen mit gewöhnlichen Schwimmern entgegenzuarbeiten, distinguieren sie sich durch ihre äußere Erscheinung (kurze, oft blondierte Haare, häufig Tätowierungen, bei den Männern sind häufig die Beine rasiert, alle tragen auffällige Uhren) und ihre Verhaltensweisen: durch ausgiebige Dehn- und Aufwärmübungen, neben dem Becken liegende wasserfeste Trainingspläne, auf die nach jeder Bahn ein Blick geworfen wird, durch - oft nur flüchtige - Begrüßungen und Verabschiedungen und nicht zuletzt durch die - sehr individuell gehandhabten - langen Trainingseinheiten. Die Triathleten fallen also dadurch auf, dass es ihnen gelingt, innerhalb der Schwimmhalle einen exklusiven Raum zu inszenieren, der eine Aura verbreitet, die sie als etwas Besonderes aus der übrigen Szenerie heraushebt.

Den Wettkämpfen kommt im Triathlon eine zentrale Bedeutung zu. Denn erst hier werden die im Training getrennt absolvierten Schwimm-, Rad- und Laufstrecken unmittelbar aufeinander folgend zurückgelegt. Das setzt wiederum besondere Orte voraus, deren herkömmliche Nutzungen für die Zeit der Wettkämpfe eingeschränkt werden müssen. Triathlon-Wettkämpfe müssen bei der Polizei und dem Tiefbauamt angemeldet werden, Straßen müssen gesperrt, Fahrradständer, Umkleidemöglichkeiten, Organisations- und Verpflegungsstände müssen aufgebaut werden. Die Triathlon-Praxis integriert sich also nicht - wie beispielsweise das Inlinehockey - in die alltäglichen Nutzungsformen öffentlicher Orte, sondern macht umgekehrt solche Orte für kurze Zeit (und nicht ohne Schwierigkeiten und organisatorischen Aufwand) zu Sonderbereichen des Sports.

Für die Teilnehmer haben die Wettkämpfe den Charakter besonderer Ereignisse. Wettkämpfe von überregionaler Bedeutung werden als Events (vgl. Gebhardt et al. 2000) inszeniert und finden an besonderen Orten statt. Solche Events werden von den Triathleten wegen ihrer besonderen Atmosphäre und ihres >Mythos` geschätzt: Man nimmt lange Anreisewege, teure Hotelzimmer und hohe Startgebühren in Kauf, um beim Triathlon in Ratzeburg oder beim Ironman in Roth dabei zu sein. Hier kann man sich dann von den die Strecke 
säumenden Zuschauern beklatschen und aufmuntern lassen. Abschließend taucht man in die - wie Detlef versichert - »sehr ausgelassene Stimmung im gesamten Zielbereich«.

Triathlon kann sich nicht auf feste Räume stützen und dadurch auch keine räumliche Exklusivität festsetzen. Konkrete Räume und überdauernde Raumstrukturen haben hier nur sekundäre Stützfunktion. Zum überwiegenden Teil müssen die Sporträume in der Praxis stets aufs Neue hergestellt und aus dem Alltagsleben herausgelöst werden.

\section{InLinehockey: Der verflüssigte Raum}

Der traditionell als Wochenmarkt genutzte Platz im Herzen eines zentralen Berliner Innenstadtbezirks ist exemplarisch für die neuen Spiele. Er hat etwa die Größe eines Fußballfeldes und ist von verkehrsberuhigten Spielstraßen, Bänken und Bäumen umsäumt. Mittwochs und samstags ist hier Wochenmarkt. Neben den berlin-typischen türkischen Gemüseständen bieten dann viele Ökobauern aus dem Umland, italienische Feinkostläden, Bienenwachskerzenhändler und Falafelverkäufer ihre Waren an: Angebote für ein in dieser Gegend recht gut vertretenes, besser verdienendes links-liberales Publikum im Alter zwischen 30 und 50 Jahren, das hier mit Marktbesuchern aus anderen sozialen Milieus zusammentrifft. Auf begrenztem Raum stoßen disparate Lebensstile aufeinander: die »Streetcorner Society« (Whyte I996) vorwiegend Jugendlicher und junger Männer türkischer Herkunft, daneben schwarz gekleidete Philosophiestudenten, aber auch die alte proletarische Eckkneipe: ein urbaner Mikrokosmos, angefüllt mit Bildern und Gefühlen großstädtischen Lebens, Bühne des Essens und Trinkens, Nahrung für das Ohr und vor allem für das Auge.

Wenn kein Markt ist, wird der Platz nach Schulschluss von Kindern und Jugendlichen mit BMX-Rädern, Inlineskates oder Skateboards genutzt. Aufgrund seiner glatten Oberfläche ist er außerordentlich gut dafür geeignet. An fast jedem Nachmittag und an allen Sonntagen treffen sich hier aber auch Jugendliche und Erwachsene zum Inlineskaten. Der Platz wird dann - nicht immer konfliktfrei - aufge- 
teilt: Auf der einen Hälfte mischen sich blutige Anfänger mit virtuosen Könnern, die wie Balletttänzer auf Rollen ihre atemberaubenden Pirouetten drehen. Auf der anderen Hälfte spielen vornehmlich junge Männer Inlinehockey, zumeist bis es dunkel wird. Zusätzlich finden hier regelmäßig kommerzielle Inlineskating-Anfängerkurse der Firma K2 statt. »Was ich eben ganz schön finde, ist, dass es eben so'n buntes Miteinander ist, Große, Kleine, Junge, Alte«, sagt Uwe, der zum harten Kern der Inlinehockey-Spieler auf dem Platz gehört. Wer an diesem Ort spielt, möchte am »Karma« (Thomas) dieses öffentlichen Straßenraumes mit seinen Geräuschen, Gerüchen und Aromen teilhaben. Manchmal unterbricht ein Spieler seine Fahrt und setzt sich zu einem kurzen Plausch oder Flirt an einen der Cafétische. Die Spielenden nehmen die Stimmungen und Kräfte des Ortes in sich auf und geben ihre Energien umgekehrt an den Ort ab; so tragen sie zu seiner Lebendigkeit bei. Das Spiel fügt sich in das lokale soziale Getriebe ein. Der Ort reflektiert das Selbstverständnis der Gruppe, die sich hier zum Spielen trifft. »Im Sommer ist es ein bisschen wie im Süden, es ist ein Anlaufpunkt [...] und so etwas wie ein sozialer Brennpunkt, [...] so Schwulenzentrum und ganz nette Lokale drum herum«, erläutert Alexandra.

Im Unterschied zu künstlichen Sportanlagen ist dieser Raum organisch in das Großstadtleben eingelassen. Er hat keine scharfen Grenzen: Wie selbstverständlich durchqueren Passanten mit Einkaufstüten oder Kinder mit Fahrrädern und Bobbycars immer wieder die Spielräume der Skater; permanent müssen diese ihren Spielball unter geparkten Autos oder den Stühlen und Tischen der angrenzenden Straßencafés hervorangeln. Gerade dieses Ineinander von Spielraum und Großstadtleben macht den Reiz des Spielens an diesem Ort aus. Die Akteure wollen ausdrücklich keinen regulären Platz mit festen Feldmarkierungen und echten Toren. Entscheidender ist, betont Tim, dass man während des Spiels »rasch mal 'n Pils trinken gehen und auch von Frauen beobachtet werden kann, also, 'n bisschen gesehen werden ist da auch immer wichtig«. Anders als in Sporthallen und -stadien sind die Rollen von Zuschauern und Akteuren aber nicht klar verteilt. Der Austausch der Blicke, die zugleich visuelle und soziale Verbindung zwischen denen, die spielen, und jenen, die vor den Cafés sitzen oder ihren Alltagsgeschäften nachgehen, all dies ist für 
die Akteure von zentraler Bedeutung. Physische Anstrengung, Schweiß, auch Erotik, die im traditionellen Sport in abgeschirmten Sonderräumen bleiben, werden hier öffentlich ausgestellt: Das Private wird zur öffentlichen Geste; jede Spielbetätigung erlangt einen eigenen Aufführungscharakter.

Für Karl ist das Inlinehockey-Spielen ein Angebot, das sich harmonisch in die Attraktionen einfügt, die der Kiez um diesen Platz für diejenigen seiner Bewohner bereithält, die zu genießen verstehen. Ebenso gutgelaunt, wie er bei gutem Wetter seine Skates anlegt, um sich in das Spielgeschehen zu mischen, hat er sich vorher ein Frühstück in einem der angrenzenden Cafés gegönnt. Der zentral gelegene Platz lädt ihn geradezu ein, sich von seinen Stimmungen treiben zu lassen. In den Spielpausen wählt er eine Kleinigkeit aus dem kulinarischen Angebot vor Ort, um sich dann vielleicht wieder dem Spiel zu widmen. Eventuell geht er aber auch »erst mal kurz hoch auf die Bude«, um sich zu erfrischen und sich anschließend mit anderen zum Pizza-Essen zu treffen. »Der Platz ist mein erweitertes Wohnzimmer«, sagt Karl. Ähnlich wie beim Flaneur, der die Stadt auf seinen Promenaden zu seiner Wohnung macht (vgl. Benjamin I99I), sind die Grenzen zwischen Wohnraum und urbaner Nachbarschaft fließend.

Dieser räumlichen Unschärfe, diesem Verwischen der Grenzen zwischen Öffentlichem und Privatem, korrespondiert eine zeitliche Unbestimmtheit: Das Spiel geht irgendwann los, neue Spieler kommen hinzu, andere hören auf; irgendwann ist es beendet, weil niemand mehr mitmacht oder weil es schlicht zu dunkel wird. Die Akteure folgen keiner übergeordneten Zeitordnung, sondern ihren eigenen Zeit- und Körperrhythmen. Pause wird gemacht, wenn man zu erschöpft ist; man hört auf, wenn es keinen Spaß mehr macht. Am Rand des Spiels entstehen diffuse kommunikative Zwischenzonen, weder Spiel noch Alltag, in denen sich vorübergehend kleinere Grüppchen von Spielern und Passanten zusammenfinden. Was im organisierten Sport räumlich, zeitlich und organisatorisch säuberlich voneinander geschieden ist - Sporttreiben, Essen, Trinken, Reden, Mode, Musik, Darstellung usw. - verschmilzt hier mit den Klangkulissen, Gerüchen und visuellen Eindrücken des Straßenraums zu einer dichten sinnlichen Szenerie. Sporttreiben ist im Rahmen dieser Szenerie nur noch ein - wenn auch ein wichtiges - Element unter anderen kulturellen 
Praktiken mit ihren verschiedenen affektiven und kommunikativen Funktionen.

Für Marc ist genau dies entscheidend, dass das hier »mehr ist als nur Sporten«. Im Kontrast zum klassischen Sport, in dem möglichst reine Wettkampfbedingungen geschaffen werden sollen, suchen die Akteure der neuen Spiele bewusst unreine Verhältnisse auf. Entgrenzung, Entdifferenzierung und Vermischung tragen maßgeblich zum Reiz ihrer Spiele bei. Trennungen zwischen innen und außen, Spiel und Alltag, Akteuren und Zuschauern, privat und öffentlich, Sporttreiben und anderen kulturellen Praxen werden verflüssigt; scharf einund ausgrenzende Rahmen gibt es nicht.

Im Unterschied zu den Funktionsräumen des organisierten Sports hat der öffentliche Straßenraum zahlreiche »Leerstellen« (Zinnecker i979: 744), die zur Umgestaltung geradezu auffordern. Bereits das praktische Erschließen des für andere Zwecke konzipierten Straßenraums als Spielterritorium gehört für die Akteure zum Erfahrungsreichtum des Spiels. Immer wieder kommt es zu Konflikten, z.B. zwischen den Hockeyspielern und dem erwähnten K2-Anfängerkurs. Diese werden wortlos in der Aktion ausgetragen: Die Hockeyspieler dribbeln in hohem Tempo bedrohlich nah um die Anfänger herum, verteilen sich geschickt auf dem Platz, passen den Ball von einem Gruppenmitglied zum nächsten. Irgendwann ist das Claim erfolgreich abgesteckt und wird durch das Aufstellen von Toren - in der Regel umfunktionalisierte Böcke für die Marktstände - auch symbolisch markiert. Wenn die Spieler von solchen handelnden Raumeroberungen berichten, schwingt oft ein wenig Stolz mit: »Wir schaffen uns unser Spielfeld selbst«, sagt Jo. Diese Aneignung und symbolische Umdeutung des Raums ist zugleich performativer Gründungsakt der Spielgemeinschaft. Aneignung ist dabei nicht als Okkupation $\mathrm{zu}$ verstehen, sondern als eine lebendige Aktualisierung, die den öffentlichen Raum als einen symbolischen Ort erzeugt und mit eigenem Sinn ausfüllt (vgl. Muchow/Muchow I935). Der primäre Rahmen des städtischen Raumes wird genutzt, aber durch einen in der eigenen Sportpraxis erzeugten sekundären Rahmen re-interpretiert. ${ }^{\text {I2 }}$

Wenn die Sonderräume des klassischen Wettkampfsports eine Heimstatt für die Familie der Vereinssportler bilden, dann ist der in den neuen Spielen vollzogene Ausbruch aus diesen Räumen gleichzei- 
tig ein Ausscheren aus den familienähnlichen Strukturen des Vereins ${ }^{\mathrm{I}}$. Die festen Orte, Strukturen und Regeln des organisierten Sports stellen die sozialen Beziehungen in ihrem Innern auf Dauer. Sie bilden einen schützenden und stützenden Rahmen für das Verhalten und übernehmen insofern die Funktion, die das Haus für die Familie hat (vgl. Simmel I992). Die Akteure der neuen Spiele verzichten dafür bewusst auf die Entlastung bergender und stützender Rahmen; sie wollen und müssen ihr Spiel nun selbst gestalten.

Anders als die institutionalisierten, immobilen und klar von der Umgebung abgegrenzten Sonderräume des traditionellen Sports haben die Räume der Straßenspiele keine überdauernden physischmateriellen Grenzen. Sie werden in sozialen Prozessen von den Akteuren als mobile und flüchtige Bedeutungsräume konstituiert. Die Spieler schaffen ihre Spiel- und Bewegungsräume situational ${ }^{\text {I4 }}$, indem sie sich in der Stadt auf Rollen fortbewegen und sich mit Hilfe von Attributen wie besondere Kleidung, Verhaltensweisen, Körpermerkmale als Gemeinschaften darstellen. Die konjunktive Bewegungs-, Spiel- und Darstellungspraxis verwandelt den Ort in einen Raum emotional hoch besetzter Aktionen. Indem die Spieler die räumlich-materiellen Gegebenheiten der Umgebung als Widerstände und Entfaltungsmöglichkeiten für ihr Bewegungshandeln nutzen, ist der Raum für sie kein neutrales, gleich bleibendes Medium, sondern ein von Bedeutungen erfüllter, gelebter Raum, der im Spiel praktisch konstituiert, erfahren, vielleicht auch erkannt wird (vgl. Bollnow I994). Die Akteure der neuen Spiele subjektivieren und verändern somit den öffentlichen Raum durch ihre körperliche Praxis und beanspruchen diesen dadurch zugleich gegen andere gesellschaftliche Gruppen (vgl. Dangschat I996).

\section{ANMERKUNGEN}

I Mit dieser Gegenüberstellung von früher und heute, alt und neu soll nicht der Eindruck erweckt werden, dass die vereinsförmigen Spiel- und Sportformen durch die postkonventionellen neuen Spiele einfach abgelöst werden. Vielmehr koexistieren derzeit beide Formen. Der Verein besteht allen Unkenrufen zum 
Trotz nahezu ungebrochen fort. Er ist die Organisationsform, von der sich die Akteure der neuen Spiele, folgt man ihren eigenen Aussagen und dem darin formulierten Selbstverständnis, abstoßen und unterscheiden wollen. Und er stellt zugleich den Hafen dar, in dem die Reise der neuen Spiele nicht selten endet (vgl. Lamprecht/Stamm I998).

2 Dieser Öffentlichkeitsaspekt unterscheidet die Ausbruchsbewegung der neuen Spiele auch von historisch früheren Formen, wie Eichberg (I986) sie dargestellt hat.

3 Dies ist der Fall, wenn der Sportraum und die Finanzmittel knapp sind, wenn es um Belegzeiten für Hallen geht oder wenn Fußballplätze, Eishallen oder Schwimmbäder exklusiv für den Profisport freigehalten und dem Breiten- und Schulsport vorenthalten werden. Mitunter tauchen auch symbolisch-politische Aspekte auf. So sollte das Münchner Olympiastadion mit seinen transparenten Dachflächen das neue, offene und demokratische Deutschland repräsentieren.

4 Vgl. zur »topologischen Raumwahrnehmung « in der »verinselten« Gesellschaft Zeiher (I983) und Zeiher/Zeiher (I994) sowie Heitmeyer/Anhut (2000) zur unterschiedlichen Raumwahrnehmung bei Mädchen und Jungen. Zum Einfluss der neuen Kommunikationstechnologien auf die Raumwahrnehmung vgl. Featherstone/Burrows (I995).

5 Der Raum bildet lediglich eine vernachlässigte Kategorie innerhalb der sportwissenschaftlichen Diskussion; vgl. Franke (1985) und Weis (I995).

6 Vgl. zur Ausdifferenzierung des modernen Sports neben Bourdieu (I992 und 1993) auch Eisenberg (I999) und Cachay/ Thiel (2002). Zur funktionalen Differenzierung vgl. grundsätzlich Luhmann (I997).

7 Wie Elias und Dunning (2003) zeigen, nehmen bei den Vorläufern des Fußballspiels ganze Dörfer an einem wilden, weder durch künstliche Grenzen des Raumes noch der Zeit eingeschränkten Gegeneinander teil.

8 Gumbrecht (I998) leitet daraus die Möglichkeit einer besonderen Präsenzerfahrung ab.

9 Die Stadtsoziologie hat dieser Entwicklung der sportiven Nut- 
zung von Räumen, Plätzen und Straßen bisher wenig Beachtung geschenkt. Die mit den neuen Spielen verbundene Entwicklung steht im Kontext einer »Politik der Festivalisierung« (Häußermann/Siebel ı993) des öffentlichen Raums.

Io Die Bewegung folgt somit der Logik einer Dialektik von Komplexitätsreduktion in Bezug auf das Außen und Komplexitätssteigerung in Bezug auf das Innen (vgl. Luhmann i987).

II Man könnte also von einer institutionell und räumlich gesicherten Beziehungsvorgabe (vgl. Schulze I994) sprechen, die soziale Zugehörigkeit herstellt bzw. zumindest eine Angebotsstruktur zur Gemeinschaftsbildung darstellt, diese somit räumlich absichert. Die Akteure werden damit von einem aktiven Herstellen sozialer Beziehungen und einer aktiven Aneignung von Räumen, wie es in den informellen Gruppen der neuen Spiele geleistet werden muss, entlastet.

I2 Zum Konzept der Raum-Aneignung als praktisches Erschließen vgl. auch Harms/Preissing/Richtermeier (i985: 9ff.), Nissen (1998) und Löw (2001: 249f.).

I3 Dieser Auszug in den öffentlichen Raum wird offenbar vorwiegend von Jungen und jungen Männern gesucht. Mädchen und Frauen scheinen weitaus weniger Interesse daran zu haben, sich auf öffentlichen Plätzen vor anderen in Szene zu setzen. Nach wie vor bevorzugen sie institutionalisierte Räume wie Sportanlagen, Vereine, Musik- und Ballettschulen und halten sich weniger als Jungen in öffentlichen Räumen auf. Die männlichen Akteure der neuen Spiele verlassen hingegen die Institutionen des traditionellen Sports und begeben sich statt dessen auf die Straße als einen Raum, durch den sie sich herausgefordert fühlen, um sich dort Situationen der Unsicherheit auszusetzen, an der StraßenMythologie des Abenteuers teilzuhaben und in selbst gesuchten Herausforderungen (körperliche) Exzellenz und Einzigartigkeit zu beweisen. Sie sind in ihren Spielen auf der Suche nach Heldentum, nach männlichen Tugenden und Haltungen, sie wollen Wagemut, Risikobereitschaft, Lust an Gefahr und ein selbst gesetztes Ethos der Bewährung demonstrieren (vgl. auch Stern 2003).

I4 In dieser Hinsicht haben die neuen Spielgemeinschaften einen 
wichtigen Vorläufer im subversiven Urbanismus der Situationistischen Internationale um Guy Debord und in deren strategischer Transformation des alläglichen urbanen Raumes durch die Konstruktion von Situationen (vgl. Levin I997). 



\section{Stilgemeinschaften}

Soziologische Gegenwartsdiagnosen sind nicht selten von der Klage über den Verbrauch gemeinschaftsbildender Energien, den Verlust sozialen Zusammenhalts und sozialer Verbindlichkeiten in modernen Gesellschaften getragen. So wird im Zusammenhang von Individualisierungsschüben von der Zerstörung sozialer Kohäsionskräfte, von Desintegration, Anomie, Atomisierung, kurz: von einer zunehmenden Auflösung der Gesellschaft gesprochen (vgl. exemplarisch Heitmeyer I997). ${ }^{\mathrm{T}}$

Als Kehrseite derartiger Klagen wird meist die Idee der Gemeinschaftlichkeit beschworen. Weil er Verlustgefühle bündelt, hat im Prozess der Formierung der modernen Gesellschaft seit langem der schillernde Gegenbegriff >Gemeinschaft< Konjunktur. ${ }^{2}$ Wenn Zygmunt Bauman die Postmoderne entsprechend als »Zeitalter der Gemeinschaft [...]: der Lust auf Gemeinschaft, der Suche nach Gemeinschaft, der Erfindung der Gemeinschaft, der Imaginierung der Gemeinschaft« (Bauman I992: 30I) bezeichnet, vervollständigt er nur das aus Desintegrationsbefürchtungen und der Sehnsucht nach Gemeinschaft zusammengesetzte Bild der Gegenwart.

Ein klassischer Institutionalisierungstypus solcher modernen Sehnsüchte ist die im I9. Jahrhundert entstehende Sozialform des Vereins. In Anlehnung an die Bemerkung Ferdinand Tönnies', das "Studium des Hauses « sei das "Studium der Gemeinschaft, wie das Studium der organischen Zelle Studium des Lebens« sei (Tönnies I979: 22), lassen sich an der >häuslichen< Organisationsform des 
Sportvereins gemeinschaftliche Beziehungen exemplarisch studieren. Der moderne Sport gilt von Anbeginn als eine Heimstätte für Werte und Qualitäten (Körperlichkeit, Natürlichkeit, soziale Bindungen und Gemeinwohlorientierungen etc.), die in der modernen Gesellschaft als bedroht und schützenswert erscheinen.

Wenn heute im Zusammenhang der ebenso vielfältigen wie diffusen Individualisierungsdiagnosen von einer Erosion und Gefährdung des traditionellen Vereinlebens gesprochen wird, dann allerdings nicht nur vor dem Hintergrund der Vorstellung eines Zerfalls des Gemeinschaftslebens, sondern auch in Reaktion auf eine Form von Gemeinschaftlichkeit, deren vereinsförmiger Organisation - in den Augen ihrer Gegner - etwas Konservatives und Einengendes, etwas Überholtes und Nicht-zeitgemäßes anhaftet. Während der traditionelle Sportverein aus der Perspektive postkonventioneller Sport- und Gemeinschaftsmodelle häufig als Gefährdung des Ichs erscheint, werden die neuen Spiele mit ihrer dezidierten Ablehnung der vereinsförmigen Organisierungsformen umgekehrt als Ausdruck eines radikalen IchKults wahrgenommen.

Unsere Vereins-Handballerinnen bewegen sich innerhalb dieses Diskurses. Am defensiven Duktus, mit dem sie über äußere Bedrohungen der gemeinschaftlichen Innenwelt des Vereinslebens klagen, ist dies gut erkennbar. Mit den Triathleten und vor allem mit den Inlinehockey-Spielern stehen ihnen in expliziter Abgrenzung Sozialformen des postkonventionellen Sports gegenüber, in denen sich neue Modi und Muster der Vergemeinschaftung und neue Selbstverhältnisse der Zugehörigen herausgebildet haben. Beide Gruppenkulturen zeigen neuartige Arrangements von sozialer Bindung und Individualität, die in der von Individualisierungsschüben gekennzeichneten Gegenwartsgesellschaft von besonderer Bedeutung sind. In ihren Spielen erlangen neue Ordnungsstrukturen des Sozialen eine sinnlich fassbare Gestalt. Sowohl die gemeinschaftlichen Kohäsionskräfte als auch die Intensitäten des Ichs finden hier eine Steigerung. 


\section{Hallenhandball: unter dem Dach des Vereins}

»Wir waren immer zusammen, so zehn, zwölf Frauen, immer die gleichen. Hier is' nich so 'ne Fluktuation wie heutzutage [...]. Die letzten zwei Jahre hatten wir viermal Training in der Woche und am Wochenende hatte ich ein Spiel und Ralf hat in zwei Mannschaften meistens gespielt, weil die zu wenig waren. Drei Spiele und immer die Kinder mitgeschleppt [...].« (Carola)

Fixe Spielzeiten, klar markierte Spielfeldbegrenzungen, Beitrittsformulare, Mitgliedsbescheinigungen, Beitragsordnungen, Vereinssatzungen, Spielberechtigungsausweise, Siegerurkunden, alters-, geschlechts- und leistungsbezogene Segregation, vom Verband festgelegte Reglements: den von den Handballerinnen gebildeten sozialen Zusammenhang umgibt ein komplexes, formales Rahmenwerk. Die Spielerinnen gehen regelmäßig einmal im Jahr ins Trainingslager, sie fahren an den Wochenenden zu Wettkämpfen, sie treffen sich im Vereinslokal, gelegentlich auch zu Discobesuchen und Ausflügen, und sie kommen anlässlich gegenseitiger Einladungen zu Geburtstagen und Hochzeiten sowie mindestens zweimal wöchentlich zum Training zusammen. Viele sind, wie Henriette, bereits von Kindheit an mit dem Verein verwachsen. »Also ich kenn jede Sporthalle in Berlin, die riesen Geräteräume mit den Matten und so, da konnte man dann spielen, das sind alles so Kindheitserinnerungen, da haben wir dann rumgetobt, während irgendein Spiel stattfand.«

Vor dem Training treffen die Spielerinnen nach und nach vor der Sporthalle ein. Man steht in einem allmählich größer werdenden Kreis herum; einige rauchen noch schnell eine Zigarette. Schon in diesen immer wieder ähnlichen Eröffnungssequenzen des Zusammenseins wird die Verbindlichkeit der Mannschaftszugehörigkeit deutlich. Zwischen fein abgestuften Begrüßungen, die vom einfachen > Hallo <über den Handschlag bis hin zur herzlichen Umarmung mit Küsschen reichen und die interne Differenzierung der Gruppe zeigen, werden hier immer dieselben zentralen Themen verhandelt: das Spiel vom letzten Wochenende, der nächste Gegner, vor allem aber die Absagen fürs heutige Training - ein zentrales Thema des Vereinssports. Wer fehlt, muss gute Gründe dafür vorbringen können; ihre Triftigkeit wird von allen diskutiert und beurteilt. »Die Jüngeren zeigen halt ein- 
fach kein Engagement mehr«, klagt Ina. »Die leben einfach nicht für den Handball. Die planen ihren Urlaub nicht ums Handball herum. Ich meine, wenn ich ein Spiel habe, dann fahre ich halt am Wochenende nicht weg. Und ich sag mal, wenn nicht gerade ein Todesfall in der Familie ist, dann kommt man - höchstens, wenn man Geburtstag hat, und selbst dann [...].«

Verletzungen, Schwangerschaften und Geburten werden zwar als Gründe für eine Unterbrechung der sportlichen Praxis akzeptiert, sie gelten jedoch nicht als Rechtfertigungen, um dem Vereinsleben fern zu bleiben. »Kontakt hatte ich auch in der Schwangerschaft«, sagt Henriette, »ich war öfter beim Training zugucken oder hab selber Training gemacht. Bei den Spielen war ich regelmäßig da, hab Kampfgericht gemacht oder auf der Bank gesessen.«

Der Verbindlichkeit des Vereinslebens korrespondiert die Geschlossenheit der Mannschaft, die das Handballspiel verlangt. Es zentriert sich weitestgehend auf die beiden Torräume. Von seltenen Schnellangriffen abgesehen, wird das Spielfeld dazwischen im geordneten Nacheinander von verteidigender und angreifender Mannschaft überbrückt. Die Spielerinnen formieren sich vor dem Wurfkreis zur Abwehrkette. In der sie konfrontierenden Angriffskette wandert der Ball von Glied zu Glied. Es folgen aufeinander bezogene Bewegungen der geschlossenen Ketten, bis durch Verschiebungen oder überraschende Richtungswechsel eine Lücke zum Torwurf gerissen werden kann. Ein solcher Durchbruch wird in sblinder< Kooperation vom Mannschaftskollektiv herausgespielt. Für einen kurzen Augenblick exponiert sich mit dem folgenden Wurfversuch dann eine Einzelne.

Handball ist kein Sport der äußeren Attribute. Formen der Selbstpräsentation und Selbststeigerung, die in den >ästhetischen < und postkonventionellen Sportpraxen im Vordergrund stehen, erscheinen den Spielerinnen gekünstelt und gespreizt, sie werden entschieden abgelehnt. »Handball ist keine Sportart, wo ich jetzt so auf mein Äußeres achte«, sagt Claudia, »das hat nichts mit Ästhetik in dem Sinne jetzt zu tun, sondern mit Kraft und auch mit Gegenhalten und, ick sag mal, mit Aufeinanderpralln von Körpern.«

Schon das Reglement fordert in Farbe und Schnitt einheitliche Wettkampfkleidung. Indem sich die Spielerinnen und die unterstützenden Zuschauer vor dem Spiel die Vereinsfarben in die Haare 
sprühen, werden solche Markierungen von Zugehörigkeit und Verbundenheit weiter ausgearbeitet und verdichtet. Dazu kommen Beschwörungen des Kollektivgeistes wie Abklatschrituale, Sprechgesänge mit call-and-response-Struktur oder das gemeinsame Trinken aus einem großen Kanister.

Bei den Punktspielen wird deutlich, dass die Mannschaft nur den Nukleus der über sie hinausreichenden Vereinsgemeinschaft formt. Kinder, Eltern, die Männer der Spielerinnen und Vereinsmitglieder aus anderen Mannschaften bilden ein regelmäßiges Publikum mit familiären Umgangsformen. Die Erwachsenen fragen die Kinder immer wieder nach der Bedeutung der Feldmarkierungen oder Schiedsrichterzeichen und machen sie auf diese Weise spielerisch mit den Regeln und Gepflogenheiten dieses Sports vertraut. Vor dem Spiel imitieren die Kinder die Aufwärmübungen der Mütter. Nach dem Spiel und in den Pausen erobern sie sofort das Spielfeld, die Tore und die Bälle. Dabei fühlen sich die Erwachsenen für alle umherlaufenden Kinder gleichermaßen verantwortlich, halten sie auf, wenn sie das Spiel zu stören drohen, trösten, scherzen und ermahnen. Von der Mannschaft wird die enge Beziehung zu ihrem Publikum nach dem Spiel regelmäßig körperlich beglaubigt. Hand in Hand vereinigen sich die Spielerinnen nun noch einmal vor ihren Zuschauern zur Kette. Sie reißen johlend dreimal zusammen die Arme hoch, bis dieses spontane Ritual in gegenseitiges Beklatschen übergeht.

Für die Handballerinnen ist die Anwesenheit ihrer Männer bei den Punktspielen und ihre Teilnahme am Vereinsleben eine »positive Unterstützung, weil wir dann eben eine richtig feste Truppe sind«, versichert Ulrike. »Wir sitzen danach eben auch in der Kneipe und haben miteinander alle richtig Spaß, wo das einfach eine richtige große Familie ist.« Für sie ist das Vereinsleben Teil des Familienlebens und das Familienleben Teil des Vereinslebens: eines ergänzt und stützt das andere. »Das bedeutet zum Beispiel, dass sämtliche Geburtstage von jeder Mannschaftskameradin Gemeinschaftsereignis sind «, betont Birgit.

Im Anschluss ans Training und besonders nach den Spielen am Wochenende steht für die Akteure regelmäßig >Geselligkeit`auf dem Programm. Das Vereinsleben setzt sich im immer selben Stammlokal fort. Die Atmosphäre hier erinnert an einen großen Familienausflug. 
Die Kommunikation mit dem Kellner ist vertraut und herzlich. Zwischen den Begrüßungen mit Handschlag erkundigt er sich nach den Spielergebnissen. Er kennt im Voraus die Bestellungen und spielt mit diesem Wissen. Die Kinder behandeln das Restaurant wie einen großen Spielplatz. Sie haben ihr eigenes Ritual entwickelt, durch das sie die Vereinsgemeinschaft bekräftigen. Unter dem Tisch versuchen sie immer wieder, die Schnürsenkel der Erwachsenen zusammenzubinden.

Die formalen Rahmungen der Vereinsgemeinschaft korrelieren mit einer hierarchischen Binnenstruktur. So nimmt beispielsweise die Trainerin eine zentrale Position ein, die Subordination verlangt. Sie ordnet Trainingseinheiten an, entscheidet über die Mannschaftsaufstellung und vermittelt zwischen Mannschaft und Verein. Das gelingt nicht immer ohne Streitigkeiten, die während der regelmäßigen Mannschaftssitzungen gelegentlich auch schärfer werden können. Dann »kotzt jede jetzt wirklich das raus, was ihr gerade einfällt«, berichtet Heike. Zum Vereinshandball gehört aber die ganz grundlegende Bereitschaft, »sich unterzuordnen und auch gruppendienlich zu agieren, auf dem Spielfeld und auch außerhalb«, hält Claudia fest. Neuzugänge müssen sich nicht nur in die verbindliche formale Rahmenstruktur, sondern auch in die informelle >Geselligkeit< einfügen können. »Da wird schon eine Auslese getroffen, dass man eben sich gut versteht. Das wird neben dem sportlichen Teil sogar noch 'n bisschen höher angesiedelt«, sagt Carola: »Leute, die nun permanent immer fehlen, wenn der Hamster mal hustet oder die Oma Geburtstag hat, also denen sagt man irgendwann dann auch mal, >was kommst du denn überhaupt noch?< So Tourismus da bei uns, das geht nicht.«

Handball trägt Züge einer durch Stetigkeit gekennzeichneten Lebensweise. Mit seinen festen Orten, Strukturen und Regeln stellt der Verein das Gemeinschaftsleben in seinem Inneren auf Dauer. Er ist darauf ausgerichtet, diese Innenwelt $\mathrm{zu}$ schützen und $\mathrm{zu}$ verteidigen. Die Vereinsgemeinschaft stiftet und verlangt Verbindlichkeit. Sie fordert die Anpassung und Einordnung des Einzelnen. Dafür verspricht sie als Gegenleistung Sicherheit, die von den meisten Vereinssportlern auch ausdrücklich gesucht wird. Ihnen ist gerade die institutionell garantierte Beständigkeit wichtig: das regelmäßige Training, 
der verbindliche Wettkampfkalender, die Vereinsfeiern mit ihren ritualisierten Geselligkeitsformen, insgesamt das Zyklische, das dem Leben eine vorhersehbare, gleichmäßige und feste Form verleiht.

\section{Triathlon: Gemeinschaft der Individualisten}

»Beim Triathlon ist das so, dass die Leute hauptsächlich alleine trainieren [...], weil jeder dann so seine Lücke im Tagesablauf nützt, um seinen Sport zu betreiben. Das ist dann eben sehr individuell. [...] Triathlon ist vielleicht sogar die Sportart mit der höchsten Scheidungsrate. Und das ist auch kein Wunder bei dem Trainingsaufwand, den man haben muss.« (Dirk)

Mit dem traditionellen Sport hat Triathlon die formalen Organisationsstrukturen von Verein und Verband gemeinsam. Charakteristisch ist jedoch das ausgeprägt instrumentelle Verhältnis, das viele Triathleten zu diesen Organisationsformen haben. Oft wird der Sinn einer Vereinsmitgliedschaft lediglich darin gesehen, Zugang und Trainingsmöglichkeiten in den Schwimmhallen $\mathrm{zu}$ bekommen. Viele Triathleten nehmen nur das Schwimmtraining vereinsmäßig in Anspruch und betreiben die beiden anderen Disziplinen Radfahren und Laufen alleine oder - sofern sich die individuellen Zeitpläne koordinieren lassen - mit einem Trainingspartner.

Im Unterschied zu den meisten Vereinssportarten werden auch die Wettkämpfe selten als Konkurrenzen zwischen verschiedenen Vereinsmannschaften ausgetragen. Im Triathlon steht vielmehr die Konkurrenz in fein differenzierten, durch den Verband festgelegten Altersklassen im Mittelpunkt. Deutlich manifestiert sich die individualistische Unterhöhlung der Sozialform des Vereins im Verzicht auf die aus dem Vereinssport bekannten Feste und Versammlungen, die der Pflege und Intensivierung der Gemeinschaft dienen. Für ein solches Vereinsleben jenseits der sportlichen Praxis fehlen oft bereits die entsprechenden Räumlichkeiten wie Vereinsheime oder angestammte Gastwirtschaften. Während diejenigen Akteure, die ehrenamtlich Vereinsfunktionen übernommen haben, immer wieder über die mangelnde >Geselligkeit< der Triathleten klagen, konstatieren andere ganz nüchtern, dass die Vereinsform über ihre funktionalen Aspekte hi- 
naus »für die Kontakte untereinander eigentlich gar keine Bedeutung hat« (Lena). Eine traditionelle Vereinsgemeinschaft wird überwiegend als >Vereinsmeierei< abgelehnt.

An die Stelle der durch den Verein gegebenen institutionellen Rahmungen rücken hier andere Aspekte. Neben den Vereinsstrukturen lässt sich im Triathlon ein zweiter Modus der Vergemeinschaftung erkennen, der auf die postkonventionellen Sportformen hinweist: Die Gemeinschaft der Triathleten kommt ganz wesentlich auch über ein Wechselspiel des Zeigens und Akzeptierens von Attributen zustande. Triathleten erkennen und akzeptieren sich insbesondere an ihrer Kleidung, ihrer Materialausstattung und ihren Körperformen als ihresgleichen. »Triathlon ist immer so 'ne ganze Ecke Selbstdarstellung und Körperkult«, bekräftigt Jürgen. Zu den wichtigsten gemeinschaftskonstitutiven Attributen zählt das Sportgerät: die Fahrräder, die sich durch ihr futuristisches Design selbst von einem modernen Mountainbike deutlich unterscheiden, sind überwiegend teure Hightech-Produkte einschlägiger Hersteller, die ständig mit technischen Veränderungen und Verbesserungen aufwarten. Die Wettkämpfe dienen auch der Ausstellung und Begutachtung der >Bikes<, deren jeweilige Werte, technische Eigenschaften und Besonderheiten von den Akteuren auf ihre Eigentümer übertragen werden. »Man ist immer beschäftigt mit dem Material. Es ist auch in den Köpfen der Leute eben eine spannende Sache. Man guckt eben, was fährt denn der für'n Rad, wenn man da so durchgeht vor dem Wettkampf, wo dann alle abgestellten Räder stehen. Wer fährt was, und ach, das ist ja das Rad von dem Lothar Leder oder von dem Jürgen Zeck, ${ }^{3}$ ah toll, sieht einfach toll aus«, berichtet Dirk. Neben den Fahrrädern zählen windschnittige, >spacige< Helme, teure Sonnen- und Schwimmbrillen (»Oakley«), Zeitfahr- und Laufanzüge (»Desoto«), Thermoanzüge, spezielle Lauf- und Fahrradschuhe, Armbanduhren und Pulsmessgeräte zum exklusiven Equipment.

Ein besonders wichtiges Attribut, durch das die Zugehörigkeit zur Gemeinschaft der Triathleten erworben und angezeigt wird, sind die Körperformen. Die Sichtbarkeit der Muskulatur wird durch die möglichst knappe, enganliegende Sportkleidung unterstrichen. Oft wird sie noch durch eine wohlplatzierte Tätowierung, ein Piercing oder durch das Abrasieren der Körperbehaarung betont. Werden über diese 
zugehörigkeitsrelevanten Attribute und ihren distinktiven Wert einerseits die exklusiven Grenzen der Triathlon-Gemeinschaft aufgewiesen, so stilisieren sich die Triathleten - im Unterschied etwa zu den Mitgliedern einer Vereinsmannschaft mit ihren einheitlichen Trikots über die je individuellen Kombinationen und Variationsmöglichkeiten dieser Attribute zugleich als ausgesprochene Individualisten: Die Triathleten kommen zusammen, um ihr »eigenes Ding (Jürgen) zu machen. Dies kommt schon in den üblichen flüchtigen Begrüßungen und dem immer wieder beobachteten Verlassen der Schwimmhalle ohne Verabschiedung zum Ausdruck. Die geringe Bedeutung der Siegerehrung bei Wettkämpfen ist ein weiterer Hinweis: Während sich meistens nur die von der Ehrung betroffenen drei Erstplatzierten der jeweiligen Altersklassen für diese Zeremonie interessieren, haben sich die anderen schon längst auf den Nachhauseweg begeben, um möglichst schnell ihre im Internet veröffentlichten Zeiten und Platzierungen zu studieren.

In der folgenden, von Lena berichteten Episode, wird der individualistische Zug der Gemeinschaft der Triathleten exemplarisch deutlich: Die Triathleten, die sich regelmäßig zum Rad- und Lauftraining treffen, befolgen vor allem ihre je individuellen Pulsvorgaben. »Die haben ihre Richtwerte, und ob die sich jetzt nun mit dir treffen, um zu laufen, oder ob die alleine laufen, ist eigentlich egal. Es kommt vor, dass du in einer Gruppe zusammen losläufst und plötzlich merkst du, jemand zieht an und läuft weg. Und du wunderst dich die ganze Zeit, ohne dass der sagt, was eigentlich los ist. [...] und dann läuft der weg, läuft übers Feld, kommt plötzlich ganz schnell zurück, dann denkst $\mathrm{du}$, was ist denn hier los, bis du nach einer halben Stunde erfährst, der läuft nach seinem Puls gerade, weißt du? Und die Leute sagen ja nicht irgendwie, >lasst uns doch mal jetzt zusammen<, sondern die machen dann ihr Ding und puh, das ist denen egal, ob du jetzt da bist oder nicht, die können auch allein laufen, denen ist das nicht wichtig, dass du in der Gruppe zusammen was machst.« Obwohl Lena in dieser Sequenz die mangelnde Gemeinschaftsorientierung der Triathleten beklagt, wird in ihrer Erzählung gleichzeitig deutlich, dass gerade im extremen Selbstbezug eine Gemeinsamkeit der Akteure liegt: Der ohne Ankündigung Weglaufende kommt genauso schnell wieder zurück (nur um möglicherweise im nächsten Augenblick - seinen 
Pulsvorgaben entsprechend - wieder davonzulaufen). Die Triathleten bilden eine sporadisch entstehende Gemeinschaft individualistisch orientierter Einzelner, die zum Laufen, Schwimmen und Radfahren zusammenkommen, in der jeder für sich bleiben will.

\section{Inlinehockey: Ad-hoc-Gemeinschaften}

»Das ist ja eben auch das Schöne, dass man so spontan so 'ne Sachen machen kann, eben so 'n Freizeitsport, ohne eben groß anzuwerfen, dicke Taschen noch zu schultern, sondern eben sagen, Mensch, die Sonne kommt raus, ich spiel mal 'n Stündchen [...], vielleicht in der Rechten noch 'n Eis, kann man ja auch machen, gibt ja so 'ne Ballkünstler.« (Karl)

Können im Triathlon noch modifizierte Organisationsstrukturen des Vereins beobachtet werden, so formiert sich die von uns beobachtete Gruppe von Inlinehockey-Spielern in ausdrücklicher Abgrenzung zum Verein und unter Vermeidung jeglicher Vereinsattitüde. Dies zeigt sich nicht zuletzt daran, dass diese Gruppierung auf materielle Rahmungen, wie sie die klassischen Sonderräume des Sports bieten, bewusst verzichtet und sich ihre sportiven Räume mitten in der Stadt selbst herstellt. Unsere Inlinehockey-Spieler, vorwiegend männliche Erwachsene zwischen Ende zwanzig und Anfang vierzig, treffen sich bei annehmbarem Wetter fast jeden Nachmittag für ein paar Stunden. Diese Regelmäßigkeit wird allerdings nicht wie im Verein von einer Institution gefordert; sie folgt vielmehr dem inneren Kompass des Vergnügens. Die selbstgewählte Verbindlichkeit wird von den Akteuren nicht als Zwang, sondern als Selbstbestimmung und -verwirklichung verstanden.

Anders als im Verein verfügt die Gemeinschaft der InlinehockeySpieler über keinerlei formale Strukturen, die ihren Zusammenhalt institutionell und äußerlich abstützen könnten. Umso mehr entscheiden über die Zugehörigkeit zur Gemeinschaft informelle Faktoren: Gemeinsame Bewegungen, Verhaltensweisen, Haltungen, Gesten und ein Fundus gemeinsamer Vorlieben artikulieren, stützen und erzeugen einen Zusammengehörigkeitssinn, der von hohen emotionalen Investitionen getragen ist. Diese Attribute fließen in einem gemein- 
samen Stil zusammen, der die Akteure körperlich vergemeinschaftet und ihren Haltungen Gestalt verleiht.

Die Regelmäßigkeiten und Verbindlichkeiten liga-, tabellen- und punkteorientierter Leistungsbezogenheit werden als autoritäre körperliche Disziplinierungen abgelehnt. Die mit traditioneller Vereinskultur assoziierte >Geselligkeit< wird als spießig und verkrampft empfunden. Dagegen schätzen die Hockeyspieler, dass man »locker bleibt« und »alles nicht so eng sieht«. Das Ideal der Gemeinschaft ist, wie Sebastian propagiert, der selbstbestimmte »Großstadtsingle, der kommt und geht, wann er will«. An die Stelle personeller Kontinuität tritt ein Fluss von selbstbestimmten Zeit- und Körperrhythmen, zufälligen und flüchtigen Personenkonstellationen. Feste Freundschaften wie im Verein bilden sich kaum. »Auf dem Platz ist es ganz nett, aber mein Freundeskreis ist woanders«, sagt Carlo.

Das unverbindliche Kommen und Gehen steht allerdings nur denjenigen offen, die den Stil beherrschen, der als gegenseitiger Erkennungs- und Zugangscode fungiert. Dieser Gruppenstil wird durch Selbststilisierungen sichtbar gemacht. Die Zugehörigen präsentieren die Besonderheiten ihrer Gruppe an sich selbst, durch individuelle Darstellungen, die jeder beherrschen muss. Neben den richtigen Skates, Rollen, Kugellagern und Schlägern muss in diesen individuellen Interpretationen des Gruppenstils vor allem die Kleidung passen.

Obwohl sie den Trikotzwang des organisierten Sports verachten, lässt sich auch bei den Inlinehockey-Spielern ein gemeinsamer Kleidungsstil identifizieren. Dieser zeigt sich freilich gerade in seiner Antimode: der Abwesenheit von Markenklamotten und gestylter Sportlichkeit. Die Spieler folgen einer Ästhetik demonstrativer Unsportlichkeit und Nonchalance. Zum grellen futuristischen Outfit der Triathleten bilden sie einen größtmöglichen Gegensatz. Weite, zerschlissene Jeans, schlotternd schlaffe Shorts, ausgewaschene und abgetragene T- und Sweatshirts gelten als Ausdruck souveräner (Nach-) Lässigkeit gegenüber den als Zumutungen empfundenen Imperativen disziplinärer Leistungssportlichkeit und modischer Kleidungszwänge.

Die informelle Gemeinschaft der Inlinehockey-Spieler bildet sich in einem doppelten Wechselspiel des Zeigens und Akzeptierens. Die Akteure suchen sich eine Spielgemeinschaft, die zu ihrem eigenen Stil passt. Sie wählen die Gemeinschaft, von der sie akzeptiert werden, 
weil sie in der Lage sind, deren Stil an sich selbst zu präsentieren. Die Eingliederung erfolgt scheinbar nahtlos, durch für den soziologischen Beobachter kaum erkennbare ritualisierte Eintrittspraxen: Auf die Gruppe der Spielenden gleitet ein Skater zu, der sich eben noch ein Frühstück in einem der angrenzenden Straßencafés gegönnt hat. Er bleibt für einige Augenblicke in Distanz zum Spielgeschehen stehen, beobachtet das Spiel, hantiert prüfend mit seinem Hockeyschläger, legt seine Ellenbogenschoner an und rückt seine Knieschützer zurecht, die er über einer viel zu weiten, ausgewaschenen Jeans trägt. Auf diese Weise zeigt er den schon Spielenden alle für die Zugehörigkeit zur Spielgemeinschaft wichtigen Attribute: Skates, Schläger, Knieschoner und Kleidung, aber auch die Haltungen und Gesten, an denen die Spielenden ihresgleichen identifizieren. Ohne für den Beobachter wahrnehmbare Begrüßungen, Absprachen oder Ähnliches wird er dann ins Spielgeschehen aufgenommen.

Eine wirkliche Integration in die Spielgemeinschaft ist damit allerdings noch nicht gewährleistet. Der Novize muss in der ersten Phase des Mitspielens eine Art Bewährungsprobe bestehen. Er wird auf seine Spielweise getestet. Der informelle »Straßenkodex« der Spieler unterbindet zu große Härte. »Nur leichtes Touchieren ist o.k., aber alles, was dich hinschmeißt, ist eigentlich schon verboten«, erklärt Sebastian, »der Platz hier ist wahrscheinlich das Softeste, was du in der Richtung finden kannst, auf allen anderen Plätzen geht es viel mehr zur Sache.«Verstöße gegen diese Spielauffassung werden durch die Gruppe selbst geregelt, z.B. indem zwei bereits fest der Gemeinschaft angehörende Spieler lässig an einem neu hinzugekommenen, zu hart spielenden Akteur vorbeirollen und sich dabei für diesen deutlich vernehmbar darüber unterhalten, dass man hier so eigentlich nicht spiele. Greifen solche gruppeninternen Selbstregulationsmechanismen nicht, folgen ohne explizite Absprache andere Formen der Konfliktregulierung bis hin zum schleichenden Ausschluss: Spieler, die den »Straßenkodex« ignorieren, werden ebenso wie diejenigen, denen es am nötigen Spielkönnen mangelt, schlicht nicht mehr angespielt.

Dies betrifft häufig auch die wenigen Spielerinnen. Ihr Bemühen, ins Spiel einzutreten, ist zunächst in aller Regel erfolgreich. Das Spiel läuft jedoch mit der Zeit immer stärker an ihnen vorbei; sie haben 
kaum eine Chance, an den Ball zu kommen. Meistens ziehen sie sich nach einiger Zeit von allein zurück und verlassen das Spielfeld. Nur sehr wenige können mithalten und werden von den männlichen Akteuren als Mitspielerinnen anerkannt. Weil der »Straßenkodex« für ein eher >körperloses < Spiel sorgt - wichtiger sind trickreiche Pässe u.Ä. - können sie im Rückgriff auf grundlegende Qualitäten des Inlinehockey wie Geschicklichkeit, Eleganz und Gewitztheit eine Spielweise entwickeln, die es ihnen ermöglicht, sich zu behaupten. Für Tina beispielsweise besteht der besondere Reiz des Spiels genau darin, unter Einsatz ihrer besonderen Fähigkeiten die an Statur überlegenen männlichen Gegner - sie bezeichnet sie als »Schränke« - auszuspielen. >Männlichkeit< und >Weiblichkeit` werden in der Spielpraxis selbst modelliert. Das trickreiche Spiel einer körperlich unterlegenen Spielerin, das den kraftvollen Einsatz eines physisch überlegenen Gegenspielers ins Leere laufen lässt, gilt dann als typisch weibliche Spielweise, die aber auch von einigen männlichen Spielern praktiziert wird, denen ihre Mitspielerin Tina dann die Eigenschaften »von Natur aus ganz sanft, so zierlich und so«, zuweist.

Die Spiel- und Bewegungspraxis ist nicht nur der Kern des Spiels, sondern auch der Nukleus der Gemeinschaftsbildung im Inlinehockey. Anders als in den von außen angeleiteten Trainingsprozessen des organisierten Sports entwickeln die Akteure in wortlosen körperlichen Austauschprozessen gemeinsame Bewegungsmuster: Einer macht eine Bewegung vor, ein anderer greift sie auf, moduliert sie ein wenig, macht etwas Neues daraus. Erfahrungen der Spannung und der Erregung, des Spielflusses, des gegenseitigen Bewegungsverständnisses sind für die Zugehörigkeit entscheidend. Wer wirklich dabei sein möchte, muss an dem gemeinsam entwickelten Bewegungsrepertoire teilhaben können.

\section{Rahmung versus Attribute}

Die vorausgegangenen Beschreibungen haben hinsichtlich der je spezifischen Vergemeinschaftungsmuster unserer drei Bewegungskulturen einen Kontrast erzeugt, der sich zu einer typisierenden Entgegensetzung weiterführen lässt: Einer Gemeinschaftsbildung über 
institutionelle und materielle Rahmungen im Handballverein steht der Aufbau von Gemeinschaft über einen doppelten Auswahlprozess des Präsentierens und Akzeptierens von Attributen gegenüber, den wir im Triathlon und auf dem Platz der Inlinehockey-Spieler beobachtet haben. ${ }^{4}$

Während sich Gemeinschaft bei den Handballerinnen innerhalb fester Rahmungen aus Sporthalle, Vereinsheim, geregeltem Trainings- und Wettkampfkalender, schriftlich fixierten Regeln und Statuten usw. konstituiert, bildet sie sich bei den Inlinehockey-Spielern entscheidend über Attribute wie die Kleidung und die Spielgeräte in Verbindung mit typischen Gesten, ritualisierten Praxen und gemeinsam geteilten Bewegungen; Gemeinschaft wird von den Akteuren in eigener Regie immer wieder aufs Neue performativ hergestellt.

Die Handballerinnen interpretieren ihre Vereinsgemeinschaft als Hort der Verbindlichkeit, institutionell garantierten Beständigkeit und Sicherheit. Sie erleben sie als heile Innenwelt, die Schutz vor allerlei äußeren Zumutungen gewährt. Die neuen Spielgemeinschaften betrachten sie als negatives Gegenbild dazu: als Ausdruck eines gegenwärtig grassierenden Individualismus, der gemeinschaftliche Bande und Verlässlichkeiten zu zerstören droht. Spiegelbildlich dazu erkennen die Akteure der neuen Spiele im traditionellen Vereinssport lediglich ein Synonym für Leistungs- oder Gruppendruck, für überkommene Normierungsansprüche und individualitätsfeindliche Standardisierung. Für die von ihnen gebildeten Sozialformen nehmen sie dagegen Qualitäten wie Zwanglosigkeit und individuelle Autonomie in Anspruch. Diese gegensätzlichen und sich aufeinander beziehenden Akteursperspektiven lassen sich in der folgenden Unterscheidung zusammenfassen: Der festen, klar und formell geregelten Zugehörigkeit zum Verein stehen neue, flüchtige und offene Gemeinschaftsformen gegenüber, die nur in stets wiederholten Zusammenkünften der Zugehörigen existieren.

Unsere Explikationen der Akteursperspektiven und die daraus gewonnene Polarität sind idealtypisch. Wir folgen darin aber gleichzeitig auch der praktischen Logik der Teilnehmer. Die Vertreter beider Seiten benutzen in ihren wechselseitigen Klassifizierungs- und Stilisierungspraxen empirisch vorfindbare Realitätsdimensionen, um bereits vorgefasste Ansichten über die jeweils >Anderen< zu festigen. 
Beobachtbare Unterschiede werden von den Angehörigen beider Sportmodelle einem typisierenden Kontrastmuster eingefügt, sodass auf beiden Seiten homogene Welten entstehen, die von der Entgegensetzung gegen das jeweils andere Modell belebt werden. Dieselben Aspekte und Züge, die aus der einen Sicht die Gefährdung bewahrenswerter Ideale des verbindlich-verlässlichen Miteinanders repräsentieren, stehen aus der anderen Perspektive für die begrüßenswerte Überwindung von Einengung und Zwang. Das eine ist das Spiegelbild des anderen; innerhalb dieses Spiegelverhältnisses konturieren sich beide Seiten wechselseitig und bringen sich in diesem Sinne erst hervor.

Mit größerer Distanz zu den Teilnehmerperspektiven entsteht indessen ein anderes Bild. In der Außenperspektive wird deutlich, dass die Opposition zwischen Rahmungen und Attributen, zwischen Konstanz und Flüchtigkeit, Fremd- und Selbstorganisation nicht einfach mit der Unterscheidung von Vereins- und neuen Spielgemeinschaften zusammenfällt. Vielmehr sind Prozesse performativer Vergemeinschaftung auch für den Verein relevant, wie umgekehrt die neuen Spielgemeinschaften nicht nur im Hier und Jetzt ihrer Aufführungen entstehen, sondern immer auch auf institutionelle Rahmungen und Grundlagen rekurrieren.

\section{Die performative Erzeugung der iVereinsfamiliel}

Die Unterschrift unter eine Beitrittserklärung garantiert noch nicht die Zugehörigkeit zu jenem Gemeinschaftstypus, der im Rahmen des Vereins existiert. Das formelle Mitglied der Organisation ist noch längst nicht Teil der informellen Cliquen, die sich innerhalb des Vereins bilden und ihn mit Leben füllen. Dies hat die vorangegangene Beschreibung der Handballerinnen gezeigt. Die als Gegenbewegungen zu einer vermeintlich von Zerfall bedrohten Gesellschaft entstandenen Vereine sind unter organisationssoziologischen Gesichtspunkten zwischen Markt, Staat und Familie angesiedelt. In Sportvereinen vermischen sich arbeitsteilig-zweckrationale Strukturen, differenzierte Autoritätsebenen und formalisierte Kommunikationswege mit WirGefühl, Geselligkeit und Personenbestimmtheit (vgl. Heinemann 
I998: 89-109). Erst durch die Belebung des organisatorischen Rahmens in informellen Akten der Vergemeinschaftung kann der Verein von seinen Mitgliedern als gemeinschaftliche Gegenwelt zur modernen Gesellschaft mit ihren Antinomien und Spaltungen erlebt werden.

Die Spielerinnen machen Neulingen klar, dass die Vereinsgemeinschaft eines über die formale Mitgliedschaft weit hinausreichenden Engagements bedarf. Das Gemeinschaftsleben muss durch kontinuierliche zeitliche und emotionale Investitionen lebendig gehalten werden: »Also wenn man sich mannschaftlich bindet, sollte man auch damit einverstanden sein, gewisse Sachen zu verschieben oder Prioritäten zu setzen. Wichtig ist, dass ein Neuer dann danach in der Kabine auch irgendwas erzählt und nicht nur sitzt, sich anzieht und abhaut [...], dass man auch mal was besorgt, wenn Feten gemacht werden, oder Geburtstagsgeschenke [...]«, fordert Silke. Das eingeforderte Engagement ist mit Vergrößerungsversprechen und mit Teilhabe am symbolischen Kapital des Vereins verbunden. Wenn an das Engagement für den Verein appelliert wird, dann wird dies mit der Tradition, mit seinem guten Namen oder mit seiner Verankerung in den Familien der Zugehörigen begründet. Oft waren bereits die Eltern oder gar die Großeltern Mitglieder. Der Verein fungiert als Kristallisationspunkt der familiären Lebenswelt. In familieninternen Gesprächen oder beim gemeinsamen Anschauen von Fotoalben werden herausragende Spiele, Vereinsfeste und andere Ereignisse erinnert. Das Vereinsleben stellt ein Archiv an Bildern, Ereignissen und Personen bereit, aus dem die Familie schöpfen kann, um eine gemeinsame Erinnerung zu arrangieren oder zu stabilisieren. Wie die Familie den Verein stützt, indem sie ihn über die in ihr tradierten Erinnerungsgesten lebendig hält, wirkt umgekehrt auch der Verein als Katalysator für den familiären Zusammenhalt.

Analog zur Familie kann auch der Verein einen geschützten Innenraum erzeugen, in dem sich seine Mitglieder entfalten können. Diejenigen, die im Verein einen Resonanzboden für ihre Interessen, Neigungen und Wünsche finden, steigern durch die Teilnahme am Vereinsleben ihre Subjektivität. Für sie ist der Verein eine besondere Welt, in der sie von den anderen wahrgenommen und anerkannt werden. Sie engagieren sich für den Verein, weil dieser ihnen etwas be- 
deutet und ihnen für ihr Engagement etwas zurückgibt. Sie bringen >Opfer<, die belohnt werden.

Gerade weil er familiäre Strukturen und Kommunikationsformen innerhalb seines institutionell-organisatorischen Rahmens fortsetzt, kann der Verein aber auch in Konkurrenz zur Familie treten. Das von den Mitgliedern mit Hingabe hergestellte und aufrecht erhaltene Gemeinschaftsleben im Verein muss dann in doppelter Abgrenzung nicht nur zum öffentlichen Leben, sondern auch $\mathrm{zu}$ ihrem privaten Familienleben ausbalanciert werden: Durch >gesellige< Aktivitäten nach Training und Wettkampf, Ausflüge mit Vereinskollegen, Geburtstags- und Weihnachtsfeiern mit der Mannschaft etc. darf die Familie, »das zweite Privatleben, das man ja nebenbei auch immer noch hat« (Monika), nicht beeinträchtigt werden.

Die von den Vereinsmitgliedern vollzogene Belebung gemeinschaftlicher Bande gegen jene kalte, individualisierte Welt, der die Akteure der neuen Spiele zugerechnet werden, gelingt gerade deshalb, weil auch hier, wenngleich in anderer Form als in den neuen Spielen, performative und informelle Elemente aufgenommen werden. Unter dem Dach des Vereins gedeiht eine Vielfalt informeller Zusammenkünfte und gemeinschaftsstiftender Rituale. Mit ihrer Hilfe werden >warme< emotionale Bindungen zwischen den Mitgliedern erzeugt, beglaubigt und immer wieder erneuert.

Auf diesen performativen Handlungsebenen entscheidet sich mit der Herstellung gegenseitiger Anerkennung auch der Grad der Zugehörigkeit zur >Vereinsfamilie $<$. Während sich die Einzelnen in ihrer formellen Mitgliedsrolle gleich(gültig) sind, werden auf der Ebene des informellen Miteinanders »Auslesen« (Carola) getroffen. Integration und Selektion, Inklusion und Exklusion gehören zusammen. Es werden praktische Unterscheidungen vollzogen zwischen denen, die zum inner circle gehören, jenen, die sich nur an der Peripherie des Vereinslebens befinden, und solchen, die die Harmonie der >Vereinsfamilie stören. Auf dieser informellen Ebene entstehen Anpassungsdruck (bis hin zum Gruppenzwang), situative Machtasymmetrien und interne >Hackordnungen<.

Die Vereinsgemeinschaft ist eine bergende, schützende und belohnende, aber auch eine kontrollierende und normalisierende Einrichtung. Mitunter reicht es aus, »die falsche Unterwäsche zu tragen« 
(Carola), um die Zugehörigkeit in Frage zu stellen. Solche Abweichungen werden in der Intimität der Umkleidekabine sensibel registriert. Abwertungen und Ausgrenzungen folgen der Logik eines >guten Drinnen und >schlechten Draußen<. Im Rahmen eines solchen Deutungsmusters personifizieren Spielerinnen, die sich in der Wahl ihrer Kleidungsattribute allzu deutlich von den anderen unterscheiden wollen, die von der Gemeinschaft abgelehnten Werte einer bedrohlichen Außenwelt; sie sind in einem bestimmten Sinne un-heimlich.

Die kontrollierenden und normalisierenden Wirkungen des Vereins gehen von den informellen Aktivitäten innerhalb seiner formalen Strukturen aus. Körperliche Nähe und Transparenz füreinander, wie sie unter der Dusche oder bei längeren Auswärtsfahrten im Bus entstehen, sind hierfür von entscheidender Bedeutung. Die für »körperbetonte soziale Systeme« (Rittner I983) wie den Sportverein typische Öffnung der Person gegenüber den anderen, die sich auch im obligatorischen Duzen äußert, hat ihre Kehrseite in gegenseitiger Überwachung und Kontrolle.

Bildet das informelle Vereinsleben mit seiner gemeinschaftsstiftenden körperlichen und emotionalen Nähe die gegen-moderne Seite des Vereins, so werden in den zweckrationalen und formellen Strukturen demgegenüber seine modernen Dimensionen deutlich. Die Sozialform Verein steht damit nur mit einem Bein in jener urbanen Moderne, die Georg Simmel in seinem berühmten Aufsatz über Die Großstädte und das Geistesleben charakterisiert hat. Simmel zeigt hier, dass das Ordnungsgebilde der Großstadt die Einzelnen davon entlastet, sich mit »den Tiefen der Persönlichkeit« (Simmel ı998: I20) in die Beziehungen mit anderen einbringen $\mathrm{zu}$ müssen. Vereinfachungen und Typisierungen gestatten vielmehr einen >oberflächlichen Austausch, in den die Menschen nur mit Teilen ihrer Person eintreten, während vieles den anderen verborgen bleibt. Als formale Organisation und rechtlich verfasstes Ordnungsgebilde ist der Verein den »Tiefen der Persönlichkeit« gegenüber in ähnlicher Weise indifferent. Prinzipiell kann jeder, unabhängig von seinen persönlichen Merkmalen, Attributen, Einstellungen, Glaubenshaltungen und sonstigen Vorlieben, die formelle Vereinsmitgliedschaft erwerben. In die für das empirische Vereinsleben konstitutiven informellen Aktivitäten ist die Person hingegen weitaus umfassender involviert. Für die Teilnahme 
an diesen Aktivitäten spielt die »Tiefe der Persönlichkeit« eine entscheidende Rolle. An diesem gegen-modernen Innenleben des Vereins kann der Einzelne nur als >ganze Person partizipieren - hier steht er auch als >ganze< Person auf dem Prüfstand.

\section{Die Rahmungen der neuen Sportszenen}

Wie sich die Vereinsgemeinschaft nicht ausschließlich über feste Rahmungen und Satzungen bildet, so erzeugen sich die neuen Spielgemeinschaften umgekehrt auch nicht nur im Hier und Jetzt flüchtiger Aufführungen. Zwar fehlen ihnen die institutionell-organisatorischen Strukturen des Vereins, aber auch sie kommen mit Hilfe von Rahmungen zustande. Ihr sozialer Zusammenhang gründet auf ähnlichen verkörperten Dispositionen der Teilnehmer, und ihre Selbstorganisation rekurriert auf ein fremdorganisiertes, von der Sport- und Lifestyle-Industrie bereitgestelltes Angebot (vgl. auch Heinemann I998: 86). Dazu zählen nicht nur die aus Sportgeräten, Equipment, Accessoires und Kleidung geschnürten style packages, sondern auch Körperbilder und Stilvorgaben in Video-Clips und Special-InterestMagazinen.

Die neuen Spiel- und Sportszenen sind von der Vereinsgemeinschaft also nicht dadurch unterschieden, dass hier die Rahmungen gänzlich fehlen. Sie lassen sich vielmehr durch eine im Vergleich viel stärkere Gewichtung der Attribute und durch eine andere Qualität ihrer Rahmungen charakterisieren. Betrachten wir unter diesem Blickwinkel nochmals die bereits skizzierte Szene des Eintritts eines Akteurs in das Inlinehockey-Spiel: Der Spieler zeigt, indem er, ausgestattet mit den richtigen Attributen, auf die bereits Spielenden zufährt, dass er in die Gemeinschaft aufgenommen werden möchte. Er geht davon aus, dass die Spielgemeinschaft $\mathrm{zu}$ ihm passt. In einem stummen und spontanen Akt beschließen gleichzeitig auch die Spielenden, dass der Neue konvenieren könnte. Im Spiel schließlich wird seine Akkreditierung zur von allen bekräftigten Zugehörigkeit. Dieser Vorgang beruht auf einem doppelten Selektionsprozess: Ein Neuer wird gewählt, weil er bereits im Vorhinein die Attribute gewählt hat, die zum Stil der Gemeinschaft passen (Gebauer/Wulf I998: 256ff.). Seine 
Wahl ist offenbar von einem bereits erworbenen »inneren sozialen Schiedsrichter« angeleitet worden. Denn, »selbst wenn man als >Stilist< allein ist, posiert man vor einem sozialen Spiegel « (Soeffner 200I: 84).

Das geschilderte Geschehen verläuft deshalb so reibungslos, weil es sich innerhalb einer »prästabilierten Harmonie« (Bourdieu 200I: I83) zwischen dem einzelnen Stilisten und der Spielgemeinschaft vollzieht. Beide Seiten gehören schon zusammen, bevor sie zum ersten Mal aufeinandertreffen; >rein kommen die, die drin sind < lautet das Prinzip dieser neuen Spiel- und Sportszenen (vgl. Schmidt 2002: I42ff.). Die schon vorab existierenden Übereinstimmungen beziehen sich dabei nicht nur auf äußere Merkmale wie Kleidung, Accessoires und Sportgeräte, sondern vor allem auf den im Habitus verankerten praktischen Umgang mit den äußeren Attributen sowie auf die in mimetischen Übungsprozessen und im Spielen selbst erworbene »Gemeinschaftsmotorik« (Gebauer 2002: I62ff.). Die Konkordanz der Akteure zeigt und bestätigt sich in ihren Gesten, Haltungen und Bewegungen. Die Spieler benutzen ihre Skates und ihre Schläger wie »Musikinstrumente« (Karl), um im Zusammenspiel gemeinsame Empfindungen und Fähigkeiten auszudrücken. Entscheidend sind eine richtige Schlägerführung, das ökonomische und symbiotische Zusammenwirken von Körperkräften und reibungsarmen Kugellagern sowie virtuose Bewegungstechniken, die den Körper »vielfältig von Kopf bis Fuß«(Karl) in Anspruch nehmen und einem im Vollzug stets aufs Neue bekräftigten Prinzip gleitend-fließender Geschmeidigkeit folgen.

Die Gemeinsamkeit hat eine von allen geteilte motorische Basis: Sie entsteht aus der Leidenschaft für ganz bestimmte Bewegungsweisen. In den von den Mitspielern geteilten Bewegungen finden die Spieler einen Resonanzboden, der die in den Tiefenschichten des Körpers aufbewahrten Emotionen und Erinnerungen in Schwingung versetzt. In den Bewegungen der Einzelnen, aus denen sich diese Gemeinschaftsmotorik zusammensetzt, ist in emblematischer Verdichtung das Kollektive enthalten: der gemeinschaftskonstitutive Stil der Mühelosigkeit, in dem sich die Ablehnung starrer Organisationsformen buchstäblich verkörpert. Die Bewegungen des Fließens und Gleitens grundieren eine Körpermetaphorik, in der die für die Ge- 
meinschaft spezifischen Selbstbilder der Offenheit und Toleranz, aber auch die der Besonderheit und des Außergewöhnlichen performativ bekräftigt werden. Die sinnliche Kollektivität flüssiger Spielzüge und Bewegungsweisen überwindet sichtbar und spürbar feste Formen, verkrustete Strukturen und >Hausordnungen $<.5$

Durch die Figurationen und Bewegungen des Spiels wird das Kollektiv kohärent und für sich selbst anschaulich. Im Zusammenspiel von Bewegungsmustern, Zeichen, ritualisierten Handlungen, Gesten und Symbolen formieren sich kollektive Repräsentationen und ein gemeinsamer Glaube (vgl. Durkheim I9I2). In den körperlichen Aufführungen im Spiel modelliert die Gemeinschaft ihre Gestalt; sie wird erkennbar und beginnt, für sich und andere sozial zu existieren. ${ }^{6}$

Ein eigener Stil bildet den Kern der kollektiven Identität, die in den Prozessen körperlicher Vergemeinschaftung geschaffen wird. Es ist der von allen Beteiligten anerkannte Stil, der die Gemeinschaft zusammenhält, ihr Beständigkeit gibt und ihre zeitliche Kontinuität sicherstellt.' Als ein solcher Stabilitätsgarant tritt in den neuen Spielgemeinschaften der Stil an die Stelle von Satzungen und Vereinsstrukturen. Er bildet den Nerv der Gemeinschaft, dessen Lebendigkeit ständig erhalten und erneuert werden muss. Die Ästhetik und Stilistik der Personen macht ein institutionelles Gerüst überflüssig.

Weil Zugehörigkeit über geteilte Bewegungen, Codes und Selbstdarstellungen erworben wird, in denen sich ein gemeinsamer Geschmack artikuliert, sind diese Spielgemeinschaften hinsichtlich der habituellen und stilistischen Merkmale ihrer Akteure recht homogen. Das Fehlen formaler Kriterien für die Aufnahme, »diese Lockerheit, dieses >natürliche Sich-Finden « und Informelle« macht aus ihnen »exklusive Gemeinden« (Krais 200I), in denen die »Treue zum je angesagten Stilbild« als Inklusionskriterium im Grenzfall »sogar rigoroser gehandhabt [wird] als die klassische Vereinsmitgliedschaft « (Richter 200I). Die auf der Basis der Gemeinschaftsmotorik entstehende Interkorporalität, die ein wechselseitiges, gleichsam intuitives oder praktisches Verstehen möglich macht, hat mit stilistischen zugleich soziale Grenzen (vgl. Meuser 2002: 38ff.). Im Gegensatz zu den Selbstbildern multikultureller Offenheit, die in den Interviews entworfen werden, ${ }^{8}$ ist aus der Beobachterperspektive nicht nur eine große Homogenität in Bezug auf die Geschlechtszugehörigkeit, sondern 
auch die ethnische Herkunft der Spieler zu erkennen: Es sind überwiegend deutsche Männer aus den Mittelschichten, die hier spielen.

Die Treue zum Stil bezeichnet eine Form und Qualität sozialer Bindungen, die gerade unter Individualisierungsbedingungen besondere Relevanz erhält. ${ }^{9}$ Während Attribute und der Modus körperlicher Vergemeinschaftung im Sportverein allein für die Bildung informeller Gruppierungen innerhalb des festen Rahmenwerks Bedeutung haben, wird die körperliche Vergemeinschaftung bei der Bildung von Stilgemeinschaften zentral; die institutionellen und organisatorischen Strukturen sind demgegenüber von untergeordneter Bedeutung. An die Stelle von Mitgliedsausweisen tritt die Belebung einer gefühlten Zugehörigkeit in wiederkehrenden Zusammenkünften. Unsere Inlinehockey-Spieler reihen sich in dieser Hinsicht in eine Pluralität von Stilgemeinschaften ein, die ein neuartiges Phänomen sozialer Aggregation und Kohäsion darstellen, das in die von Individualisierungsprozessen am stärksten betroffenen Milieus der Mittelklassen vorrückt.

\section{AnMerkungen}

I In der Sportsoziologie finden diese Klagen insofern Widerhall, als ein allgemein schwindendes Interesse am Verein festgestellt oder das Organisationsverhalten der Jüngeren problematisiert wird, die sich angeblich immer seltener ehrenamtlich in Institutionen und Gemeinschaften wie Parteien, Gewerkschaften, Kirchen und dem Sportverein engagieren würden. Es wird ein demgegenüber neuer Typus des »Sporthoppers« identifiziert, der im Sinne »frei flottierender Bindungsfähigkeit« zwischen den vervielfältigten Modellen und Angeboten des Sports hin und her wechsle: »Montags Tai Chi, dienstags Jogging im Wald, mittwochs Fußball und am Wochenende Bauchtanz in der Männergruppe« (Bette I999: I68). Vgl. kritisch zum >Sporthopping< auch Baur/Burrmann (2003).

2 Die Begriffe >Gemeinschaft< und >Vergemeinschaftung< werden hier mit Bezug auf Max Webers Unterscheidung von »Vergemeinschaftung « und Vergesellschaftung« verwendet. Es wird 
damit ein spezifischer Modus der Erzeugung sozialen Zusammenhalts durch kulturelle Praktiken bezeichnet, die sich durch die Kopräsenz der Teilnehmer auszeichnen. Demgegenüber zielt der Terminus »Vergesellschaftung « auf die formal-rationale, vermittelte, über die ortsgebundenen Vergemeinschaftungen hinausreichende Ebene des Herstellens sozialer Beziehungen durch Arbeitsteilung, Tausch, Verträge, Verbände usw., vgl. Weber (I980: 2I-23).

3 Lothar Leder und Jürgen Zeck gehören $\mathrm{zu}$ den besten deutschen Triathleten.

4 Die Leitkategorien »institutionelle Rahmungen« und »Attribute« haben wir von Nakane (I972) übernommen und unserem Forschungskontext adaptiert.

5 Für Bauman erhält das Flüssige sogar die Bedeutung einer »leading metaphor for the present stage of the modern era« (Bauman 2000: 2).

6 Eine Gruppe muss, »um für sich und für andere zu existieren [...], Vorstellungen über sich vermitteln, dramaturgische Akzentuierungen [...] ihrer relevanten Merkmale, eine Art Stilisierung, die zur Bildung des kollektiven Glaubens beiträgt, ohne den die Gruppe kein Anrecht auf soziale Anerkennung hat« (Boltanski I990: 50).

7 Schon die Forschungen des Centre for Contemporary Cultural Studies haben diese gemeinschaftskonstitutiven Funktionen des Stils deutlich gemacht: »The creation of a distinctive style is not simply a matter of embodying the subculture's own identity and self-image: It also performs the function of defining the groups' boundaries more sharply in relation both to its members and all outsiders, a function which has particular consequences for the groups' continued existence« (Clarke: I976: I82). Vgl. zur Relevanz des Stilbegriffs für Prozesse der Gemeinschaftsbildung auch Hahn (I986) und Soeffner (I986, I995, 200I).

8 »Das ist hier kunterbunt gemischt. Also hier sind sämtliche Völkergruppen vertreten und Deutsche natürlich auch dabei, und die vertragen sich perfekt, obwohl es natürlich so ein körperbetonter Sport ist, wo Emotionen auch mal blank liegen, aber es ist sehr harmonisch, muss ich sagen«, erzählt Carlo. »Es ist 
gemischt, es hat auch damit zu tun, wer hier wohnt, gerade bei den Kids«, erläutert Sebastian. »Hier so Andreas und so, die sind so I3, I4, die wohnen da in der Ecke. Andreas ist glaube ich Ukrainer, dann gibt's noch so ein paar, weiß ich nicht, aus Jugoslawien glaube ich einer, etwa ein Querschnitt, so wie sie in Berlin auch wohnen, aber Türken z.B. gar nicht. Ich glaube, die sehen das nicht mal, da gibt es auch nur Fußball und BMW oder Mercedes.«

9 In der soziologischen Diskussion gelten solche Stilgemeinschaften gemeinhin als posttraditionale Sozialformen, die zwar als Reaktion auf die Erosion traditionaler Bindungen und Gemeinschaftsformen entstünden, denen es aber - weil sie einen lediglich ephemeren und instabilen Charakter und eine nur schwache soziale Kohäsion aufweisen würden - nicht wirklich gelänge, das im Zuge der Individualisierung der Lebensverhältnisse entstandene Vakuum an sozialen Beziehungen wieder zu füllen; vgl. Schulze (I993: 459ff.), Hitzler (I998) und Keupp (1997). Geblendet von einem zu grell beleuchteten Kontrast zu traditionalen Gemeinschaften und Milieus bleiben die besonderen kohäsiven Qualitäten dieser neuen Sozialformen in dieser Sichtweise unbemerkt. 


\section{Gleiten, Rollen, Schweben}

Ohne eine hoch entwickelte Sporttechnologie wären die neuen Spiele nicht denkbar. Ob Inlineskaten, Skateboarden, BMX-Radfahren oder Gleitschirmfliegen: Es sind maßgeblich die innovativen Techniken der Sportgeräte und der Materialien, die den Akteuren die charakteristischen Bewegungsformen und Körpererfahrungen erschließen und deshalb die Faszination der neuen Spiele mit ausmachen. ${ }^{\mathrm{I}}$

Auf den ersten Blick mag es zwar scheinen, dass auch die neuen Sportgeräte nicht viel mehr sind als zweckmäßige technische Ausrüstungen, die es erlauben, auf einem harten Untergrund zu gleiten, auf Bänke, Treppengeländer, Bordsteine zu springen, darüber zu rutschen oder durch die Lüfte zu schweben. Wenn die Akteure aber über ihre Sportgeräte sprechen, bemerkt man schnell, dass nicht allein deren funktionale, sondern auch ihre ästhetischen, kommunikativen und emotionalen Qualitäten für sie Bedeutung haben: Ihre Skates, Skateboards, BMX-Räder oder Gleitschirme sind affektiv aufgeladen und in komplexe Deutungshorizonte eingewoben. Die neuen Sportgeräte sind Medien mit Kultstatus: Sie gestatten den Akteuren, ihre Körper $\mathrm{zu}$ erweitern, neue Bewegungsdimensionen $\mathrm{zu}$ erschließen, andere Beziehungen zur Umgebung, zu den Mitspielern und zu sich selbst einzugehen. ${ }^{2}$

Die neuen Sportgeräte ermöglichen aber nicht nur neue Bewegungstechniken und -erfahrungen, sie haben nicht nur vielfältige mediale und symbolische Qualitäten. Im praktischen Gebrauch der neuen Spielgeräte zeigt sich vielmehr ein verändertes Verhältnis von 
Körper und Technik. Hier realisiert sich ein Anschmiegen der Technik an den Körper und umgekehrt. Dieses wird schon in der organischen Gestalt der nach dem Prinzip der »Body-Geometrie« (Trillitzsch 200I: 2I7) geformten neuen Sportgeräte manifest. Aber auch in der Alltags- und Arbeitswelt vollzieht sich diese Verkörperlichung der Technik: Küchengeräte, Handys und Computer nehmen längst körperliche Formen an.

\section{Techilk als Gegen-Stand}

Das Verschmelzen von Körper und Technik unterscheidet die neuen Spiele deutlich vom klassischen Sport und speziell vom Turnen als eine seiner Kerndisziplinen. Zwar sind auch Turngeräte, wie die Entwicklung des Turnpferdes seit dem späten Mittelalter zeigt (vgl. Göhler/Spieth I989, Nickel I999), zunächst zum freien Ausprobieren von Dreh-, Kreisel- und Sprungbewegungen entstanden, jedoch hat sich ihre Funktion im Zuge der Pädagogisierung der turnerischen Bewegung seit den Philanthropen an der Wende vom i8. zum I9. Jahrhundert gewandelt. >Reitmaschinen $<$ Schwebebalken, Klettergerüste, verschiedenartige Kombinationen von Stangen, Leitern, Balken und Seilen, Armhebel und Armkraftmesser, Reck, Bock, Kasten und Barren dienen nun der pädagogischen, auf einem naturwissenschaftlichen Wissen basierenden und sich analytisch-synthetischer Übungsmethoden bedienenden Formung, Technisierung und Rationalisierung des Körpers und seiner Bewegungen (vgl. König I989).

Die neu entwickelten Turn- und Übungsgeräte sind in dieser Perspektive weder Produktiv- noch Destruktivkräfte, weder Maschinen noch Waffen, sondern Mittel zur Weitergabe, Einübung und Unterstützung von erwünschten Körperidealen, Bewegungs- und Verhaltensmustern (vgl. Eichberg I984: II5). Sie können Vergegenständlichungen kulturell tradierter und gesellschaftlich erwünschter Bewegungen und Körpernormen verstanden werden. ${ }^{3}$

Die stets mit spezifischen Übungsmethoden, körperpädagogischen Funktionsräumen, Diskursen, Ausführungsvorschriften, Regelwerken und narrativen Dimensionen verknüpften materiellen Arrangements der Geräte ${ }^{4}$ sind auf bestimmte Formen des Sich-Be- 
wegens, Laufens, Turnens und Spielens bezogen. Turngeräte wie das Reck oder der Barren begegnen dem Lernenden zunächst als Hindernisse, als Gegen-Stände im wörtlichen Sinn. Aufgrund ihrer Beschaffenheit verlangen diese Geräte beim Üben der an ihnen vorgesehenen Bewegungen eine durchgehende Straffung und Spannung des Körpers; sie fordern eine männlich-militärische Körperform ein. Ein entspannter, weicher Körper fügt sich an diesen Geräten unvermeidlich Schmerzen zu.

In den Übungen ordnen sich die Bewegungen nach und nach der objektiven Logik der Geräte unter. Die Geräte reihen sich entsprechend ein in eine »politische Ökonomie des Körpers« (vgl. Foucault I977), die unter Ausnutzung seiner Gelehrigkeit seine Bewegungen kultur-, klassen- und geschlechtsspezifisch strukturiert, kontrolliert und effizient macht. Eingefügt in institutionalisierte körperpädagogische Dispositive zielen die Turngeräte auf Disziplinierung und Habitualisierung, auf die Vermittlung einer ganzen Kosmologie von Werten und Wahrnehmungsweisen.

Wie im Turnen sind auch in den meisten anderen traditionellen Sportarten die materiellen und technischen Eigenschaften der Sportgeräte und Ausrüstungsgegenstände primär Mittel zum Zweck: Sie begünstigen die Entwicklung erwünschter Bewegungs- und Körpertechniken, dienen der pädagogischen Formung eines körperlichen Habitus oder der Steigerung quantitativ messbarer Leistungen. ${ }^{5}$

\section{Technik als Selbststeigerung}

In den neuen Bewegungsspielen von Inlineskatern, Mountainbikern oder BMX-Virtuosen kommen technische Sportgeräte zum Einsatz, die es den Akteuren ermöglichen, sich vom kodifizierten Bewegungsrepertoire der traditionellen Sportarten abzusetzen. Einige dieser Sportarten wie das Inlineskating haben historische Vorläufer: Bereits im I9. Jahrhundert liefen in den europäischen Metropolen und Modebadeorten Aristokraten und Angehörige des wohlhabenden Bürgertums auf speziellen »skating-rinks« Rollschuh. Die >bessere Gesellschaft< sprach von einer regelrechten »Rinkomanie«, und die Industrie entwickelte zahlreiche Rollschuhmodelle. Die »skating-rinks« 
waren mit Orchester, Restaurationsbetrieben und Zuschauerräumen ausgestattet und schufen damit die Voraussetzungen für die Inszenierung des so genannten »Gesellschaftslaufens« (Norden I998: 22f.). $\mathrm{Zu}$ Beginn des 20. Jahrhunderts gab es eine zweite Welle der Rollschuhbegeisterung. Nun wurde nicht mehr nur in den »rinks« Rollschuh gelaufen, sondern auch - ermöglicht durch Rollschuhe mit größeren Rädern - auf offener Straße. ${ }^{6}$ Während das historische Rollschuhlaufen in den öffentlichen Räumen des späten I9. und frühen 20. Jahrhunderts eher einem Flanieren auf Rollen gleichkam, ist das heutige Inlineskaten demgegenüber ostentativ sportlich geprägt: Die in den Sonderräumen des Sports entwickelten körperlichen Attribute der Sportlichkeit treten aus diesen heraus, verbinden sich mit neuen, durch die Skates möglich gemachten Bewegungsweisen und werden in dieser Verbindung in der urbanen Öffentlichkeit aufgeführt und inszeniert.

Die innovativen bewegungsgenerierenden Wirkungen und Potenziale von Rollen, Kugellagern, Skates und Boards sind zugleich in dicht geknüpfte, symbolisch-ästhetische Netze aus Popkultur, Lifestyle, Fashion, Körperkult und Expertendiskursen verflochten. Bilder und Texte der zahlreichen, größtenteils aus den Spielgemeinschaften selbst hervorgegangenen Special-Interest-Magazine (Inlineskating, Boardstein, Onboard, mountainbike downhill, freedom, Surfers, aber auch der Lifestyle-Zeitschriften wie The Face, $i$-d magazine, style oder blond) erzeugen eine eigenständige Mythologie der neuen Spiele, in der die Spitzentechnologie der Geräte einen konstitutiven Platz einnimmt. Zeitschriften wie Inlineskating widmen sich neben der »history« der Sportart, den Stars der Szene oder den besten »locations« ausführlich auch den Fahrtechniken und den technischen Qualitäten des Materials. In elaboriertem Fachjargon ist die Rede von »shock-absorptionsystems«, »Air-Zoom-Fußbetten« und »Glasfaserframes«, von einem perfekten »tuning «, das »biedere Stiefel« zu »aggressivem stuntstuff « verwandelt. In den Technik-Rubriken dieser Zeitschriften werden »Stahlfederdämpfer« oder »Diskbreaks« sowie Dutzende von Rollenmarken für unterschiedliche Einsatzmöglichkeiten, hergestellt aus 300 »chemischen Geheimrezepten« in 24 Größen, 2I Härtegraden (Durometer) und 20 verschiedenen Geometrien unter die Lupe ge- 
nommen (vgl. Inlineskating I997; Inline I997; mountainbike downhill 200I: 22ff. und 38ff.).

Die mit technischen Details gespickten Produktpräsentationen statten die Sportgeräte mit einer kultischen Strahlkraft aus und schreiben sie in die Wunschökonomie der potenziellen Käufer ein. Für die Akteure der neuen Spiele annoncieren sich die neuen Sportgeräte somit sowohl als Träger eines distinkten Selbstbildes wie als Medien von Zugehörigkeit. Hightech-Skates, Mountainbikes, Surfboards oder Gleitschirme fungieren als Artefakte der Selbststilisierung und als Mittler sozialer Beziehungen; sie tragen dazu bei, »Gemeinden der Gefühle« (vgl. Soeffner 2000: I98-203) entstehen zu lassen.

Durch warenästhetische Inszenierungen und massenmediale Präsentationen stimuliert, besetzen die Akteure die Sportgeräte mit ihren Affekten, Gefühlen, Wünschen und verwandeln sie in charismatische Produkte. In ihren symbolischen Dimensionen repräsentieren die neuen technischen Sportgeräte im Selbstverständnis der Akteure ein ganzes Bündel von Eigenschaften: Freiheit, Flexibilität und Dynamik, Abenteuerlust und Risikobereitschaft, Differenz und Anderssein, Unkonventionelles und Nonkonformes. ${ }^{7}$ Die technischen Artefakte avancieren so zu Attributen der Auszeichnung und des Auserwähltseins: sie sind Zeichen eines Kreises von Gleichgesinnten, der sich durch sie gegenüber anderen Gruppen hervorhebt und zugleich in seinem inneren Zusammenhalt gestärkt wird.

In unseren Untersuchungen finden wir ein solches, über die Technik vermitteltes Elitebewusstsein besonders ausgeprägt bei den Triathleten: »Mein Rad, das motiviert mich noch zusätzlich«, erklärt Uwe: »Ich fahr so'n Softride, und das ist eben, man sitzt da drauf, und man ist eben von der Technik her dem ein oder anderen voraus, weil man da einfach das Glück hatte, auch einen Beruf zu haben, in dem man ein paar Mark mehr verdient und sich dann eben auch ein teureres Rad leisten kann. Mit einem besseren Rad kann man eben da und dort noch ein bisschen Zeit sparen, noch rausholen, ob das nun im Ziel nachher wirklich so wichtig ist, das ist eine ganz andere Frage, aber so vom Gefühl her ist es einfach toll. Ich setz mich auf mein Rad und es macht mir einfach Spaß, wenn ich's angucke, und ich find's einfach geil, wenn ich da drauf sitze und durch die Gegend rase, weil irgendwo die Geometrie des Rades, die stimmt.« 
Durch ihre teuren, futuristisch gestylten und sich ständig wandelnden Fahrräder fühlen sich die Triathleten insbesondere gegenüber dem, Peter zufolge, »sehr konservativen Völkchen« des Radsports überlegen: »Es gibt andauernd Innovationen im Triathlon, in der Regel immer im Radbereich, dass es immer neue technische Sachen gibt, und dann denkt man, >Mensch, vielleicht könntest du dir das noch mal anschaffen<. Also das ist eben der Unterschied, bei den Radfahrern, die fahr'n hundert Jahre das gleiche Fahrrad, und in hundert Jahren wahrscheinlich auch noch, das merkt man jetzt gerade bei der Tour de France, wo das Zeitfahren wieder so langweilig gemacht wird, weil alle das gleiche Rad fahren müssen; das macht dieses Zeitfahren langweilig, weil alle hier die gleich Gurke fahr'n.« Gleichzeitig markieren die Räder individuelle Unterschiede innerhalb der Triathlon-Gemeinde und zeigen die jeweilige Stellung ihrer Besitzer an eine Gemeinschaft von Individualisten mit dem Fahrrad als wichtigstem Distinktionsmedium.

\section{Technik als Selbsterfahrung}

In den neuen Sportarten verwächst Spitzentechnologie mit dem Körper der Akteure. ${ }^{8}$ Das Sportgerät wird zu einer »leibnahen « Technik im Sinne Gehlens (I993: 94 und I48). In der Vorstellung der Akteure dienen Inlineskates der Verbesserung des menschlichen Körpers: »Der Mensch hat einen Geburtsfehler. An seinen Füßen, missbraucht zum Jogging, Gas geben oder Walken, fehlt bis tief in die goer Jahre das >körpereigene< Fahrzeug. Keinem fiel auf, dass der Mensch ein paar Rollen an den Sohlen braucht. Rollen, die ihn von vielen Zivilisationsübeln befreien: vom Schneckenspeed des Fußgängers, von der Abhängigkeit des Autos, vom Übergewicht, vom Überdruss an öden Filzballspielen [...] Darwin würde sagen: >Skaten ist ein Schritt in der Evolution $<[. ..] \ll$, heißt es im Editorial der ersten Ausgabe der Zeitschrift Inlineskating (H. I/ı997).

Die diversen Roll-, Gleit- oder Fluggeräte gehen allmählich >organische< Verbindungen mit den körperlichen Befähigungen der Spieler ein und erweitern diese. Der klassische Athlet schnallt sich nach dem sportlichen Tun sein Gerät ab; es ist nicht Teil von ihm. In den neuen 
Praktiken wachsen den Teilnehmern Räder oder Rollen an Stelle der Füße. Ein neuer Körper mit neuen Organen bildet sich heraus. Die neuen technischen Organe gehören zu den Muskelgefühlen, zur Propriozeption des Subjektes; sein Fühlraum wird vergrößert.

Die neuen technischen Geräte wirken, zumindest in der Wahrnehmung der Akteure, nicht von außen formend auf den Körper und seine Bewegungen ein, sie werden eher von innen heraus zur Entwicklung und Steigerung körperlicher Wahrnehmungs- und Ausdrucksfähigkeiten benutzt. In dieser Synthese mit dem Körper dienen sie nicht zuletzt der Stimulierung von Gefühlen: Lust, Spannung, Thrill. Durch Bewegungen des Rollens, Gleitens, Schwebens oder Fliegens werden die Sinne angereizt (vgl. auch Kemper/Sonnenschein 200I). ${ }^{9}$ Zwar ist das Erlernen neuer Bewegungen oft mit Schmerzen verbunden, zwar heben die Akteure die leidvollen Erfahrungen am Beginn ihres Lernprozesses in Erzählungen und Interviews oft ausdrücklich hervor, um das Extreme der eigenen Sportart zu betonen ${ }^{\text {IO }}$, jedoch sind die für das Rollen, Gleiten und Fliegen vorgesehenen Spielgeräte nicht wie Turngeräte starre, statische und ortsgebundene Objekte, sondern flexible Kunstglieder des Körpers, mit deren Hilfe neue Erfahrungsräume erschlossen werden und die persönliche Mobilität gesteigert werden kann.

Für geübte Spieler sind die technischen Artefakte der neuen Spiele zugleich Medien einer besonderen kinästhetischen Wahrnehmung und sensomotorischer Rückempfindungsfähigkeiten. Je höher der Könnensstand und je größer die Virtuosität, umso mehr verschwinden sie selbst aus der Wahrnehmung. Sie werden einverleibt und damit zu Sinneszonen, die die Umfänglichkeit und Reichweite des Berührens und Spürens vergrößern (vgl. Merleau-Ponty I966: I73, Beck I997: 248ff.). Der Körper expandiert, mittels Technik wird er über seine Grenzen hinaus ausgedehnt. Umgekehrt wird die Umwelt in die Bewegung und das Körperselbstgefühl einbezogen. Die Grenzen zwischen Körper und Umwelt werden im Bewegungsvollzug durchlässig, die »Gesamtwahrnehmung der eigenen Körpergrenzen lässt nach« (Habermas i999: 64).

Wo sich in den traditionellen Sportarten in der Auseinandersetzung mit der personalen und dinglichen Umwelt ein geschlossener Körper formiert und einbringt, verliert das Selbst in den technisch 
ermöglichten Gleit- und Rollbewegungen der neuen Spiele vorübergehend seine klar begrenzte räumliche Entität, ohne jedoch vollkommen in der Situation aufzugehen. Wenn das Gleiten oder Rollen beherrscht wird, ist die Person nicht ohne Halt, wie beim unbeabsichtigten Ausrutschen. Während man in diesem Fall ganz von der Situation absorbiert ist, kann sich beim gewollten und gekonnten Gleiten eine Distanz zwischen Akteur und Umgebung einstellen. ${ }^{\text {II }}$ Gespielt wird dann auf dem schmalen Grat zwischen der Auslieferung an die Situation und ihrer Kontrolle. Die Faszination der Bewegung liegt nicht zuletzt darin, diesen Schwellenzustand zu beherrschen.

Die neuen Sport- und Spielgeräte sind Medien der Selbsterfahrung und der Erneuerung von Selbst- und Weltverhältnis. Mit ihrer Hilfe können die Akteure, die sich vom konventionellen Sportverein abgrenzen wollen, ungewohnte Bewegungen explorieren, sich neu fühlen und aus den Lebensroutinen ausbrechen. ${ }^{\mathrm{I} 2}$ Diese Eröffnung neuer Möglichkeiten ist der Grund für die besonderen Beziehungen der Akteure zu den Spielgeräten: Sie ähneln denjenigen von Musikern $\mathrm{zu}$ ihren Instrumenten. ${ }^{\mathrm{I}}$ An den Inlineskates erkennen Kundige, ob jemand zum Kreis der Insider gehört. Schuhe mit Plastikschalen und Klettverschluss würden sechte< Inlinehockey-Spieler niemals tragen. Die vom Hersteller montierte Bremse wird selbstverständlich abgebaut, weil sie stört und weil Hockeyspieler aus der von uns beobachteten Subkultur seitwärts bremsen wie Eishockey-Spieler. Jedes Detail kann bedeutungsvoll werden: Die Art und der Härtegrad der Rollen ebenso wie die Schnürsenkel und die Weise, sie zu binden. Gute Rollen erkennen geübte Spieler bereits an ihrem sound. Sie werden regelmäßig gewechselt, um einen gleichmäßigen Verschleiß zu gewährleisten. Insider schnüren die Schuhe nicht bis oben, sondern lassen die obersten Ösen frei. Man hat dann, wie sie sagen, ein besseres Fahrgefühl, man kann den Fuß geschmeidiger aufsetzen; die Bewegungen werden eleganter; das Gespür für den Untergrund wird besser. Auch die Spuren, die der Gebrauch am Material hinterlässt, können Zeichencharakter annehmen. Die Abnutzung der Rollen gibt dem geübten Blick zu erkennen, wie gut jemand fährt. Scharten, Kerben und Abschürfungen sind Hinweise auf die Härte des Spiels, den Fahrstil, den persönlichen Einsatz.

Durch solche Gebrauchsspuren werden die Sportgeräte subjekti- 
viert. Es ist so ähnlich wie bei einem Gitarristen, dessen Virtuosität von anderen Musikern bereits am Zustand seines Instrumentes, an den von seinen Fingern ausgearbeiteten Kuhlen zwischen den Bundstäbchen >abgelesen werden kann. Der Gebrauch macht das technische Gerät zum Träger einer persönlichen Geschichte. Akteur und Geräte wandeln sich einander an. Im Gebrauch erwirbt der Spieler einen immer präziseren Sinn für das Gerät, der es ihm schließlich erlaubt, es so zu benutzen, als wäre es ein Körperteil. Und wie der leidenschaftliche Gitarrist sein Instrument sorgsam und an einem besonderen Ort aufbewahrt, lässt auch der engagierte Skater seine Skates nicht irgendwo herumliegen, sondern nimmt sie auch gern einmal mit ins Schlafzimmer. Als »geliebte Objekte« (Habermas I999) haben die Sportgeräte nicht nur eine Bedeutung für ihre Besitzer, sondern auch für Gleichgesinnte. Als Teil der Person verfügen sie zugleich über kulturell geteilte Bedeutungen, so dass sie zwischen der Person und ihrer kulturellen Umgebung vermitteln.

Die neuartigen Sportgeräte tragen zum Bewegungs- und Selbstgenuss der Spieler insbesondere deswegen bei, weil mit ihnen die Schwerfälligkeit und Langsamkeit des Laufens überwunden werden kann. Die Füße verlassen die Erde, diese wird nur noch mit reibungsarm gelagerten Rollen berührt, die Geschwindigkeit erhöht sich, die Arme bewegen sich wie Flügel ${ }^{14}$ und spielen mit der Kontrolle über das Gleichgewicht. Auf Inlineskates verliert die Geradlinigkeit der Vorwärtsbewegung ihre Bedeutung, die sie beim Marschieren und Laufen hat. Wichtiger wird die virtuose Bewegung um die Längsachse, das Drehen, Kurven, Kreisen, Wedeln, wie man es auch vom Snowboarding kennt. ${ }^{15}$

So vereinen die Bewegungsformen der neuen Spiele Momente technologisch unterstützter Fortbewegung (wie die durch bloßes Laufen nicht $\mathrm{zu}$ erreichende hohe Geschwindigkeit) mit einer wieder erschlossenen Nähe des Körpers zur Umgebung. Die Faszination, die diese Kombination des Ungleichzeitigen auslöst, macht auch verständlich, warum in der Zeitschrift Inlineskating nicht nur der im >Schneckentempo sich fortbewegende Fußgänger, sondern auch dessen Widerpart, das Auto, als »der natürliche Feind des Skaters« abgelehnt wird (Inlineskating, H. I/I997: I4): In den Augen der Inlineskater steht es für eine negativ bewertete Fortbewegungs- und Zivilisationstechnik, 
die den Körper bewegt, ohne dass dieser sich selbst bewegt, und ihn dabei von der Umgebung abschirmt. Zwar repräsentiert auch das Auto Geschwindigkeit und Mobilität, aber es kommt durch seine Außenwände doch zugleich traditionellen Bedürfnissen nach Abschottung und Sicherheit entgegen. Die Faszination des nicht motorisierten und vergleichsweise ungeschützten, spielerischen Gleitens, Rollens und Schwebens liegt demgegenüber darin, dass der Körper der Umgebung direkt ausgesetzt wird. Diese wird mit allen Sinnen gespürt, erfahren und praktisch bewältigt. ${ }^{16}$

Solche Erfahrungen werden jedoch gerade nicht über >natürliche< Bewegungsformen in der Natur gesucht, wie in den Lebensreformund Ausbruchsbewegungen an der Wende vom I9. zum 20. Jahrhundert, in denen Naturnähe die Verarmung der Sinneswahrnehmungen in der technisierten Großstadt kompensieren sollte. Die Skater suchen vielmehr technologisch unterstützte, gesamtsinnliche Bewegungserfahrungen in den urbanen Zentren (vgl. auch Bette I989: 63ff.). In der Bewegungspraxis wird der Großstadtraum in eine gefährliche und abenteuerliche Landschaft umgedeutet. ${ }^{17}$ Im Kontrast zu den Schutzund Sonderräumen des traditionellen Sports, die leistungsfördernd möglichst alle Störgrößen ausschalten, hält diese Landschaft (noch oder wieder) Überraschungen und Hindernisse bereit: Während der Leichtathlet die Verfälschung der Leistung durch den Wind fürchtet, weil die Zurechenbarkeit von Leistungen gefährdet erscheint, schätzt es der Inlineskater dagegen, wenn er ihm ins Gesicht bläst.

\section{TECHNIK aLS RISIKO}

In zahlreichen neuen Sportarten wird der Körper ungeschützt mehr oder minder risikoreichen Umgebungen ausgesetzt. In Bewegungen des Gleitens, Rollens und Drehens mit hohen Geschwindigkeiten, des Jonglierens mit dem Gleichgewicht und des Abhebens vom Boden beim Springen und Schweben hat das Wagnis eine andere Bedeutung als im traditionellen Sport. Denn während Risiken in diesem nur in Kauf genommen werden, um eine bestmögliche (messbare) Leistung $\mathrm{zu}$ erbringen, und es überdies in der klassischen Sportethik als verwerflich gilt, die Athleten durch zu viel oder zu avancierte Technik 
Gefahren auszuliefern, werden Risiko-Situationen in vielen neuen Bewegungspraktiken bewusst aufgesucht. Selbst in weniger risikofreudigen Sportarten wie dem Inlineskating wird noch ein Wagnis eingegangen und der Körper aus allen Haltevorrichtungen herausgenommen. Die Akteure riskieren seine Unversehrtheit. Im Gegensatz zu bewährten Mustern sportlichen Interaktionsverhaltens verzichten sie auf eine Position der Stärke und nehmen eine Position der Schwäche ein. Erworbene Sicherheiten - der feste Stand, das sichere Gehen, der solide Grund - werden mit Absicht suspendiert. Im Aufsuchen von Situationen der Verunsicherung und Überraschung tritt das handelnde Subjekt aus vorhersehbaren Bewegungsabläufen heraus.

Die Motive des Risikos (der >Sprung ins Ungewisse $<$ ), des Abenteuers und des Extremen prägen auch die Darstellungen der neuen Sportarten in den Szenezeitschriften und den Erzählungen der Akteure. Im Riskieren des Gleichgewichts und in körperlichen Suchbewegungen verlassen sie vorübergehend die eigene, gefestigte Körpergeschichte; sie gehen an die Grenzen ihres gesicherten (Bewegungs-) Könnens oder überschreiten diese sogar. Spielerisch werden die sozialen und kulturellen Bedeutungsgrenzen erprobt, die die Akteure als Habitus auch selbst durchziehen. Absichtlich derangieren die Spieler jene »vollkommene Koinzidenz« zwischen ihren im Laufe der Sozialisation inkorporierten subjektiven Schemata des Erfassens, Wahrnehmens und Bewertens und den in der Materialität und Räumlichkeit ihrer Umgebung objektivierten Strukturen, die ihnen die soziale Welt normalerweise als selbstverständlich erscheinen lässt (Bourdieu 200I: ı88). Sie spielen ein prekäres Spiel auf der Schwelle zwischen Kontrolle und Kontrollverlust; sie machen sich die vertraute Welt temporär fremd. Unter der Voraussetzung einer tiefen Vertrautheit mit ihrem Sportgerät verlassen sie die gewöhnlichen Räume und Ordnungen und suchen an deren Rändern und in ihren Lücken Schwellenzustände auf. Auf diese Weise erzeugen sie vorübergehend eine Fremdheit sich selbst gegenüber ${ }^{18}$ und lassen in diesen liminoiden Zuständen Möglichkeiten eines anderen Handelns und kultureller Innovation aufblitzen (vgl. Turner i989).

Die Risikopraxen sind von technischen Entwicklungen ebenso abhängig wie das Alltagsleben mit seinen Sicherheitstechniken. Die Suche nach Sicherheit und die Suche nach kontrollierter Verunsiche- 
rung sind in modernen Gesellschaften Komplementärphänomene (vgl. Poser 200I). Systematisch kann man zwischen solchen Sportarten unterscheiden, in denen der Körper der Technik überantwortet und ausgeliefert wird (beispielhaft beim Bunjee-Springen), und anderen Praxen, bei denen der Akteur versucht, Orientierung und Kontrolle über die Umgebung, die Technik und das Selbst (seine Ängste, die Motorik usw.) auch unter Bedingungen aufrechtzuerhalten, die ihm dies fast unmöglich machen, um mit gestärktem Bewusstsein für die eigenen Fähigkeiten aus der Bewährungssituation hervorzugehen. ${ }^{19}$ Im Vergleich zum traditionellen Sport verlagern sich die Schwerpunkte: Weitaus stärker als im klassischen Leistungs- und Wettkampfsport wird der Körper in den neuen Spielen als Instrument, Erzeuger und Empfänger von Erregung, Spannung oder (Angst-)Lust eingesetzt. In eins damit treten statt der vornehmlichen Orientierung auf die objektiv messbare Leistung die Momente der Virtuosität, der Akrobatik sowie die Fähigkeit in den Vordergrund, rasch und flexibel auf ständig wechselnde Situationen zu reagieren.

\section{TeChNiK als Mimikry}

Das Erproben, Erfahren und kontrollierte Überschreiten von Grenzen bildet für die Akteure einen Kern ihrer Bewegungspraxis. Sie lehnen alle möglichen klar ausgearbeiteten und starren Begrenzungen ab: die Trennungen zwischen Sporttreiben und anderen kulturellen Praxen, die institutionalisierten Begrenzungen von Spiel- und Bewegungsräumen, die (sportliche) Normierung und Disziplinierung des Körpers, seine Abschottung von einer als Gegner dargestellten und wahrgenommenen Umgebung und die Kodifizierung seiner Bewegungen. Die für das Rollen, Gleiten und Schweben industriell hergestellten Spielinstrumente erlauben es, diese Begrenzungen zu überwinden und neue Dimensionen eines spielerischen Bewegungshandelns zu eröffnen: Sie schaffen die Möglichkeit für eine Ausweitung der Spielund Bewegungsräume über das eigene Wohnviertel hinaus; sie führen $\mathrm{zu}$ einer Vorstellung und einem Erleben des eigenen Körpers als »offenes System « (Löw 200I: I28); und sie tragen zur experimentellen Ausdehnung eines gesicherten Bewegungskönnens bei, indem sie den 
Körpern den festen Boden unter den Füßen entziehen. Die Selbstverständlichkeit habitualisierter Bewegungsroutinen wird dann irritiert: »Alles ist ständig im Fluss, nach allen Seiten offen, es ist so wie Schweben«, illustriert Karl, einer der Inlineskater auf unserem Platz im Herzen eines Berliner Innenstadtbezirks.

In den neuen Spielen vollzieht sich ein qualitativer Wandel im Verhältnis von Körper und Technik. Durch Mimikry ans Organische verbirgt die Technik ihr Konstruktionsprinzip und minimiert Nutzungsvorbehalte. An die Stelle der klassischen Forderung an den Körper, selbst zur Maschine zu werden, ist die Anpassung der Technik an den Körper getreten. Indem sich die Technik an die Körperkräfte anschmiegt und die Empfindungsfähigkeit des Körpers steigert, unterstellt sie sich der Verfügung körperlicher Selbstkontrolle. Dadurch verlagern sich auch die Risiken ihrer Beherrschung auf die Ebene der Kontrolle der Körperkräfte. Die an den Körper angeschmiegte Technik mildert jene Skepsis ab, mit der die Menschen hypertropher Technologie begegnen. In den neuen Spielen tritt die Personalität der Sportgeräte an die Stelle monströser Apparate. Diese Personalität beglaubigt die Illusion des Menschen als Souverän der Technik, der sie zu selbstgewählten Zwecken einsetzt. ${ }^{20}$ Das technologisch ermöglichte Gleiten erweist sich als »Gesamtgestus « ${ }^{21}$ einer neuen »génération glisse« (Loret I996), die nach Erfolg und Anerkennung sucht und dabei Normen und Institutionen als Hindernisse definiert, die sie spielend überwinden will.

\section{AnMerkungen}

I Zwar gehören technische Geräte und Ausrüstungsgegenstände seit jeher zum Sport, aber in den neuen Sportarten haben sich ihre Verwendungsweise und Bedeutung grundlegend gewandelt (vgl. Eichberg I973: 8Iff.).

2 Das mögen auch die Gründe dafür sein, dass Sportarten, »in denen in besonderem Maße eine Hochtechnologie zum Tragen kommt, in den letzten fünfzehn Jahren ein überdurchschnittliches Wachstum« verzeichnen. In allen Trendsportarten lässt sich darüber hinaus eine zunehmende funktionale und ästheti- 
sche »Differenzierung der Produktpalette beobachten, so dass innerhalb »einer Klasse von Sportgeräten [...] eine Vielzahl von Erzeugnissen« konkurriert (Heinemann 200I: I8).

3 Vgl. das von der Kritischen Psychologie entwickelte Konzept der »Gegenstandsbedeutung " (Holzkamp I995: besonders 27I294). Bourdieu begreift Maschinen oder Geräte in vergleichbarer Weise als »Geschichte in objektiviertem Zustand« (Bourdieu I997: 28).

4 Foucault hat solche heterogenen, netzartig verknüpften Ensembles aus materiellen Anordnungen, Diskursen, Institutionen, architektonischen Einrichtungen, reglementierenden Entscheidungen, (Disziplinar-)Methoden, wissenschaftlichen Aussagen, Techniken, Strategien und moralischen Lehrsätzen als »Dispositive der Macht« (vgl. Foucault I978: besonders II9-I25) bezeichnet.

5 Auch bei den Hightech-Geräten und -Ausrüstungen des traditionellen Sports wie Glasfaserstäben für den Stabhochsprung oder den shark skins - Fischhäuten nachempfundene Schwimmanzüge - der Leistungsschwimmer dominiert die Funktionalität. Sie sind nur in sehr eingeschränktem Maße Gegenstand speziell auf sie gerichteter Faszination, Lust und Begierde.

6 Das Illustrierte Wiener Extrablatt vom 18.5.1909 beobachtete, dass sich in Berlin, »auf den inzwischen stark verbesserten Straßen immer mehr Angestellte der Ämter und Geschäftshäuser der Rollschuhe bedienen, um rasch aus ihren Wohnungen zu ihren Bureaus zu gelangen« (Norden I998: 34).

7 »Mein MTB [Mountain-Touren-Bike] ist mir genauso viel wert wie eine Frau«, sagt der 27-jährige Frank. »Mit dem Fahrrad hat man die letzte Freiheit auf der Straße - das ist die Anarchie des Alltags.« Frank fährt fort: »Das ist, glaube ich, die Essenz des Radfahrens. Natürlich ist es nicht ganz ungefährlich, aber das macht auch den Reiz aus, nachher sagen zu können: >Schwein gehabt<. Wenn man in der Fahrradszene drin ist, achtet man natürlich auch auf ganz andere Dinge, zum Beispiel, daß die einzelnen Teile nicht maschinell hergestellt, sondern handgeschweißt werden. Wenn ich das nötige Geld hätte, würde ich bis 
zu 7.000 DM zahlen« (die tageszeitung v. I5.6.I99I: 44; zit. nach Habermas I999: I6). Entsprechend beschreiben Snowboarder ihre Sportart im Unterschied zum »langweiligen« Schilaufen als »dynamisch«, »jung« und »frech«, als »radikal, spektakulär und eigenwillig«. Besonders beeindruckend sei die »Freiheit der Bewegung [Begrenzungslosigkeit]«: Im Gegensatz zum Schifahren gebe »es kein lästiges Querstehen oder Bremsen in den Kurven« (vgl. Marlovits 200I: 429f.).

8 »Ich merke«, so ein BMX-Radfahrer in der Szene-Zeitschrift freedom, »wie mein Fahrrad auf meine Bewegungen super sensibel reagiert, checke genau, wie das Zusammenwachsen meines Körpers und meines Standards geschieht - mein BMX wird ein Teil von mir« (freedom, H. 5/I998: 59). Zum Zueinanderkommen von Körper und Technik im BMX-Flatland vgl. Schwabe (2003).

9 »Ob Leidenschaft, Glücksgefühl oder Schmerz [...], BMX ist ein gut gemixter Cocktail, der die Sinne weckt « (freedom I998: 57).

Io Es war »wie ein Gang durch die Hölle« (vgl. Marlovits 200I: 430 und 433, Anm. 8).

II Mit einer solchen körperlich-praktisch sondierenden Annäherung an die Umgebung wird die durch die Erfindung der Dampfkraft initiierte und seither durch immer neue technische Fortbewegungsmittel forcierte Loslösung der körperlichen Bewegung aus ihrer organischen Bindung an den Umraum in Frage gestellt und nach Lösungen für eine erneuerte Annäherung zwischen Körperbewegung und Raum gesucht. Die Vereinigung von Körper, Bewegung und Umgebung wird in den neuen Sportarten aber nicht mittels >natürlicher< Körpertechniken wie dem Laufen herbeigeführt, ebenso wenig mittels ökonomisch obsolet gewordener, alter Techniken (die die Entwicklung neuer Technologien nostalgisch und irgendwie >beseelt< erscheinen ließ) wie z.B. beim Kutschenfahren der gehobenen Klassen des I9. Jahrhunderts, sondern im Gegenteil mittels eines hochtechnologisch erweiterten Körpers. Die These einer industriellen Herauslösung der (Verkehrs-)Bewegung aus ihrer organischen Bindung an den Raum hat Schivelbusch vertreten. Während die »vorindustrielle Verkehrsbewegung [...] Mimesis 
an die äußere Natur« gewesen sei, habe die Dampfkraft »dieses mimetische Verhältnis« aufgelöst (Schivelbusch 2002: I5). Zur Überführung ökonomisch veralteter Techniken in die Bereiche von Sport und Freizeit vgl. Schivelbusch (2002: I8).

I2 »Das Fahrrad«, so ein BMX-Radfahrer, »ist das Werkzeug, welches vermag, mir [...] eine Flucht aus dem Alltäglichen zu bieten, mir Spaß und Herausforderung zu bescheren und mich die Welt mit anderen Augen sehen zu lassen« (freedom, H. I/ 2002: 23).

I3 Die ersten metallrädrigen Rollschuhe wurden im I9. Jahrhundert tatsächlich von Musikinstrumentenherstellern erfunden (vgl. Norden I998: I3).

I4 In der Werbung des Skateherstellers K2 heißt es »Enjoy your flight!

I5 Die Szenezeitschrift Adrenalin portraitiert Stephane, einen 30jährigen »multiglisseur«: Er sucht »nach den schönsten Orten, um hier schöne Arabesken zu zeichnen [...]. Die Kurve ist eine in Stil und Gestik entwickelte physische Empfindung. Das Fahren von scharfen Kurven ist eine echte Droge. In der Stadt wie auf dem Wasser, auf dem Schnee oder auf dem Asphalt werden wieder und wieder die Elemente gestreichelt « (Adrenalin, H. Juni 200I).

I6 Die von Marlovits (200I: 430) befragten Snowboarder nennen drei für diesen Zusammenhang wichtige Bewegungsformen: das »Curven«, das »besonders durch seine Dynamik und Eleganz« beeindrucke, der »Cliff Jump«, der »durch Risiko und das Gefühl, ins Ungewisse zu springen« besteche, schließlich - als »unübertroffener Höhepunkt « - »das Gleiten im >Powder [Tiefschnee] [...], bei dem sich ein leichtes, fließendes, schwebendes Gefühl von Freiheit einstelle«.

I7 Der Aufbruch in unberechenbare Räume ist eine überwiegend männliche Praxis. Nach wie vor scheint sich Männlichkeit nicht nur im Aufbruch ins Abenteuer, sondern auch im spielerischen Gebrauch von Hightech-Produkten zu beweisen. Entsprechend zeigen Klaus/Pater/Schmidt (I997: 813), dass Männer und Frauen in ihrer Sozialisation unterschiedliche Haltungen zur Technik entwickeln und sich in der »Art und Weise ihrer Aneignung 
und Auseinandersetzung mit Technologien [...] als Männer oder Frauen« positionieren, d.h. ihre geschlechtliche Identität ausdrücken.

»Fremdheit macht sich auch im Bereich der Sinne geltend in Form von Abweichungen, Störungen, Beunruhigungen, von Gegenrhythmen, blinden Flecken, Echowirkungen, Heterophonien, Heterotopien und Gleichgewichtsstörungen, in all dem, was aus dem Rahmen fällt [...]« (Waldenfels ı999: I4).

I9 Wagnissport ist dann eine »Selbsttechnologie« (Foucault) zur imaginären Selbstvergrößerung. Zur Klassifizierung verschiedener Anwendungen von Technik im Wagnissport vgl. Stern (2003).

20 Die neuen Spiele weisen auf veränderte Haltungen zur Technik in bestimmten gesellschaftlichen Milieus hin. Nur ein Beispiel: Das Institut für Demoskopie in Allensbach hat im Oktober 2000 Ergebnisse einer im Auftrag des Deutschen Studienpreises/Körber-Stiftung durchgeführten repräsentativen Umfrage veröffentlicht, in der die deutsche Bevölkerung befragt wurde, welche Ängste, Hoffnungen, Einstellungen und Meinungen sie mit dem Thema Körper verbinden. Ein Ergebnis war, dass technische Eingriffe in den Körper besonders bei Jüngeren und besser Gebildeten auf große Akzeptanz treffen. 32\% hatten nichts gegen Schönheitsoperationen einzuwenden (in der übrigen Bevölkerung nur I9\%) und immerhin 22\% fänden es gut, wenn man durch den Einbau eines Gehirnchips die Leistungen des Gehirns verbessern könnte (bei Hauptschülern nur ı9\%, bei Älteren noch weniger). Technische Manipulationen am Körper werden in diesen Milieus offenkundig nicht als Bedrohung wahrgenommen, sondern als Mittel zur Selbstverbesserung: zur Perfektionierung des Aussehens, zur Optimierung der intellektuellen Leistungen, zur Verfeinerung der Sinneswahrnehmungen oder zur Intensivierung der Gefühle. Der Körper wird dann nicht als naturgegeben und schicksalhaft aufgefasst, sondern als ein Instrument und Medium, dessen Leistungs-, Darstellungsund Erlebnisfähigkeiten technologisch verbessert werden können. Die neuen Spiele lassen eine ähnliche Akzeptanz der Ver- 
schmelzung von Körper und Technik erkennen und prägen diese wohl auch selbst aus.

2I Brecht (I967: 753) versteht darunter einen »nur in vager Weise« bestimmten Ausdruck, der »der Haltung einer Epoche« physische Gestalt verleihe. 


\section{Das Brodeln im Kleinbürgertum}

Die Unterschiede zwischen dem traditionellen Vereinssport und den neuen Spielpraktiken erscheinen so groß, dass wir bei unserer Arbeit lange Zeit als geradezu selbstverständliche Annahme unterstellten, die völlig disparaten Sportverständnisse der Teilnehmer seien Ausdruck großer sozialer Unterschiede. Die an Vereinstraditionen gebundene Handballerin, den auf elitäre Exklusivität bedachten Triathleten und den alle Formalitäten und Leistungsideale des klassischen Sports ablehnenden Inlineskater hielten wir zunächst für Sozialfiguren, die völlig unterschiedlichen sozialen Klassen angehören. Wir haben, um diesen ersten Eindruck zu überprüfen, von jedem der ausgewählten Fälle ein Set von Sozialdaten erhoben: Welches ist ihr Bildungsstand, ihr Beruf und ihre aktuell ausgeübte Erwerbstätigkeit, welches ihre Haushalts- und Familienform, welchen Status haben die Eltern, wie ist also ihre intergenerationelle Mobilität einzuschätzen? Die Antworten geben Auskunft über das Volumen und die Struktur ihres Kapitals, insbesondere über das Verhältnis von kulturellem und ökonomischem Kapital. Mit Hilfe dieser Informationen ließen sich ihre Reisewege (trajectoires) im Sozialraum rekonstruieren und in ein Modell eines Raumes der sozialen Positionen eintragen (vgl. Abbildung I), ähnlich wie es von Bourdieu in seiner Untersuchung der französischen Sozialstruktur (Bourdieu I982: I95ff.) und von der Forschungsgruppe um Michael Vester (et al. 200I) für den sozialen Raum der Bundesrepublik Deutschland entwickelt wurde. 
Abbildung 1: Modell eines Raumes der sozialen Positionen nach Bourdieu (1982: 195ff.)

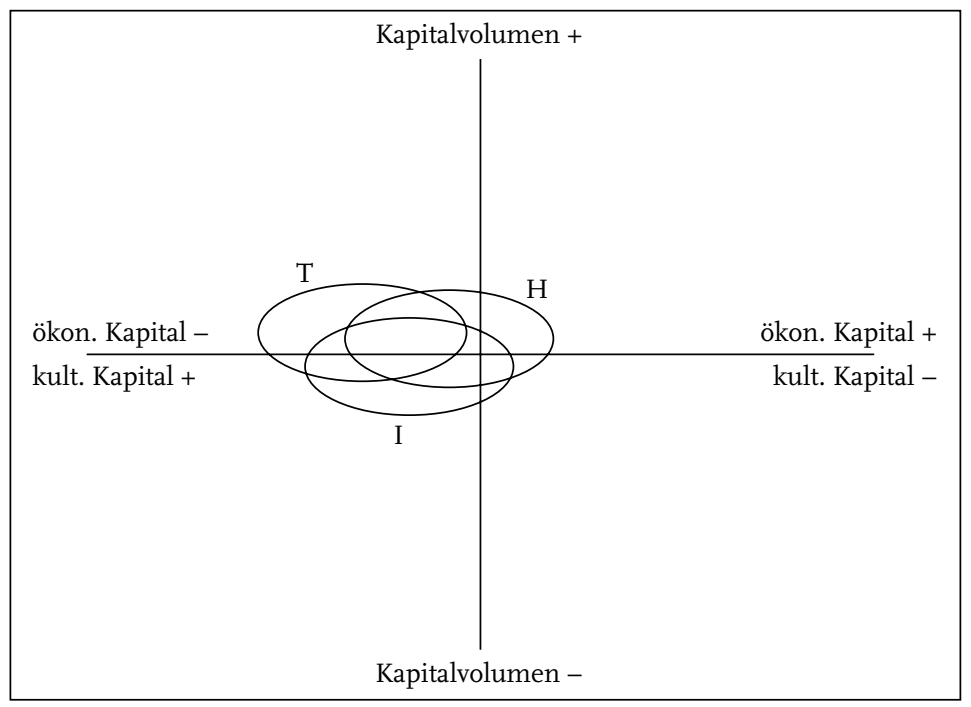

$\mathrm{I}=$ Inline Hockey; $\mathrm{T}=$ Triathlon; $\mathrm{H}=$ Handball

Die Auswertung bringt ein überraschendes Ergebnis: Zwischen den drei Sport-Gemeinschaften im Handball, im Triathlon und im Inlinehockey zeigen sich keine wesentlichen sozialen Unterschiede. Unsere Fälle sind für die jeweiligen Untersuchungsfelder zwar nicht repräsentativ, wohl aber typisch. ${ }^{\mathrm{I}}$ Unabhängig von der betriebenen Sportpraxis befinden sie sich alle in enger Nachbarschaft in einem mittleren Bereich des sozialen Raumes. Das relativ enge Spektrum der sozialen Positionen reicht von Industriekaufmännern mit Abitur, Sachbearbeiterinnen im öffentlichen Dienst mit Fachhochschulabschluss über Bankkauffrauen mit abgeschlossenem Hochschulstudium, verbeamteten Grundschullehrern bis hin zu Zahntechnikerinnen und Maschinenbauingenieuren. Obwohl wir es im Inlinehockey, im Triathlon und im Handball mit drei völlig verschiedenen sportlichen Praxen, Sportverständnissen und Lebensstilen zu tun haben, lassen sich diese Unterschiedlichkeiten nicht auf deutlich voneinander differierende Positionen im sozialen Raum zurückführen. Die Fälle aus allen drei Berei- 
chen befinden sich, auf der vertikalen Achse, auf annähernd derselben sozialstrukturellen Rangstufe. Nur in der horizontalen Dimension besetzen sie gegensätzliche und teilweise neue symbolische und kulturelle Positionen. Bisher hatten wir dazu tendiert, die Handballerinnen dem Kleinbürgertum zuzurechnen - nun mussten wir auch die Triathleten und die Inlinehockey-Spieler in deren unmittelbare Nachbarschaft einordnen. ${ }^{2}$

Dieses erste Ergebnis spricht nun keineswegs für einen schwindenden Einfluss der sozialen Lage auf das sportliche Engagement. Es verdeutlicht zunächst lediglich einen in der Sportsoziologie bekannten Befund: Die großen sozialen Klassen unterscheiden sich voneinander nicht so sehr durch die Präferenz unterschiedlicher Sportarten, sondern viel stärker danach, ob überhaupt Sport getrieben wird. ${ }^{3}$ Es gibt also gerade insofern einen starken Zusammenhang zwischen sozialräumlicher Position und sportlicher Aktivität, als Sporttreiben überhaupt gerade bei den Angehörigen der mittleren Regionen des sozialen Raumes ganz besonders verbreitet ist. ${ }^{4} \mathrm{Um}$ die besondere Verbindung zwischen den Sportpraktiken und den mittleren sozialräumlichen Regionen zu erhellen, wollen wir die widersprüchliche soziale Beschaffenheit dieses Orts in der sozialen Topologie genauer kennzeichnen.

\section{Die turbulente Mitte des sozialen Raumes}

Eine der signifikantesten Veränderungen der Sozialstruktur der Bundesrepublik seit dem Ende des Zweiten Weltkriegs ist die kontinuierliche Zunahme der quantitativen Besetzung der mittleren Lagen. Sie geht einher mit einer zunehmenden Bedeutung horizontaler Differenzierungen in dieser sozialräumlichen Region: Insbesondere die Bildungsexpansion wirkte sich nicht nur als eine »historische Drift zu mehr kulturellem Kapital innerhalb aller vertikalen Stufen der Gesellschaft« aus, sondern auch als eine »bedeutsame horizontale Bewegung vom rechten zum linken Pol des sozialen Raumes« (Vester et al. 200I: 220). In dieser horizontalen Differenzierung schlagen sich gesellschaftliche Veränderungen nieder, wie sie unter den Stichworten $>$ Tertiarisierung $<$, >Wissensgesellschaft $<$, >Wertewandel $<$ und >funktio- 
nale Differenzierung diskutiert werden - sie machen den mittleren Bereich zur dynamischsten und turbulentesten Region des sozialen Raumes. Am linken, >kulturellen< Pol der mittleren Lagen bilden sich immer wieder jugendkulturelle Avantgarden, die gegen ihre Herkunftskultur rebellieren, sich dabei zeitweilig mit anderen Klassenfraktionen zusammenschließen ${ }^{5}$ und im zeitlichen Verlauf zu einer Modernisierung der mittleren Klassenmilieus beitragen.

In der mittleren Turbulenzzone des sozialen Raumes lässt sich beobachten, dass zu einem gegebenen Zeitpunkt immer »)ungleichzeitige< soziale Formationen miteinander koexistieren oder ringen « (Vester et al. 200I: 189) - in der dynamischen Mitte kreuzen sich die Reisewege verschiedener absteigender, aufsteigender und beharrender Fraktionen. Dieses strukturelle Brodeln der mittleren Region wurde insbesondere seit den I990er Jahren noch einmal angefacht: hier haben sich seither die signifikantesten Milieu-Um- und -Neubildungen vollzogen (Vester et al. 200I: 37ff.). Dabei sind die lebensweltlich entstandenen Klassenformationen, die sich hier aufgrund des relativen Übergewichtes an kulturellem Kapital ganz entscheidend durch die kulturelle Praxis vollziehen, empirisch noch weitgehend ungeklärt (vgl. Eder 1989: 365). Am bekanntesten erscheinen noch die traditionellen kleinbürgerlichen Formationen, die aber bei genauerer Betrachtung weniger das Resultat bloßen Überdauerns über Generationen hinweg sind. Sie konstituieren sich vielmehr über immer wieder neue Abgrenzungen und Retraditionalisierungen gegenüber den angestrengt innovativen Lebensstilen in ihrer unmittelbaren Nachbarschaft. In der dynamischen Mitte des sozialen Raumes halten traditionelle Kleinbürger, die hartnäckig auf ihrem Idyll aus Familie, Haus und Schrebergarten beharren, das Spiel der Unterscheidungen ebenso in Gang wie die »Modernen Performer « (Sinus Sociovision 2002: 26ff.), die mit verschiedenen Möglichkeiten zur persönlichen Selbstverwirklichung, Stilisierung und Inszenierung experimentieren.

Im Unterschied zu den oberen und unteren Regionen des sozialen Raumes bildet dessen Mitte einen Ort »der Ungewissheit und der relativen Undeterminiertheit« (Bourdieu 1982: 537). Während die Angehörigen der oberen ebenso wie der unteren Klassen durch eine weitgehende klassenspezifische »Übereinstimmung dessen, was man ist, mit dem, was man zu sein hat« (Bourdieu I982: 53I) gekennzeich- 
net sind, tritt in der mittleren Region Sein und Scheinen oft auseinander. Hier sind die Akteure sowohl von der Kultur der unteren Klassen, die sich unter dem Zwang der Notwendigkeit gebildet hat, als auch von der dominierenden Kultur des gewandten Auftretens, der äußerlichen wie innerlichen Sicherheit, der Lockerheit und Eleganz entfernt. Von beiden Klassenkulturen unterscheiden sie sich dadurch, dass ihnen der eigene Ort fehlt, der ihr Verhalten absichern und auf den sich ihre soziale Identität verlassen könnte. In der mittleren Region des sozialen Raumes befinden sich die Akteure nicht in gewisser und vertrauter Lage, sondern in einer unbestimmten, unklaren Position, die sie dazu bewegt, sich insbesondere gegenüber den unteren Klassen strikt abzugrenzen. Komplex und widersprüchlich ist ihre Haltung in Richtung dominierende Klassen: für die einen sind diese Vorbild für mimetisches Anpassungsverhalten, während die anderen sich in eine spießbürgerliche Attitüde einigeln. Sie sind daher »unaufhörlich konfrontiert mit ethischen, ästhetischen und politischen Alternativen und gezwungen, ihre alläglichsten Handlungen in den Rahmen strategischer Entscheidungen zu stellen« (Bourdieu I982: 539). Während die strukturelle Ungewissheit in den mittleren Regionen einerseits immer neue Formen der Selbstdarstellung, »Bluff und Euphemisierungsstrategien [begünstigt], hat dies zur Kehrseite die innere Unsicherheit über die soziale Identität« (Bourdieu I982: 564).

Im Kleinbürgertum herrscht strukturell ein hoher Darstellungsund Unterscheidungsdruck. Hier treffen die ambitioniertesten Aufstiegshoffnungen von Inhabern neuer, noch nicht etablierter Positionen auf soziale Abstiegsängste in den schrumpfenden traditionellen Milieus; Glücksritter, die ständig über ihre Verhältnisse leben, sind mit rigiden Besitzstandswahrern konfrontiert. Die skizzierten vertikalen, horizontalen und diachronen Dynamiken machen die mittleren Regionen des sozialen Raumes insgesamt zu einer Zone struktureller Unbestimmtheit und Vieldeutigkeit: Hier kommt der Schere zwischen Sein und Scheinen besondere Bedeutung zu; Prozesse der Infragestellung, Umarbeitung und >Neuerfindung < sozialer Identität sind hier ständig virulent und dauerhaft ungelöst. Freilich lassen diese permanenten Um- und Neugestaltungen von Sozialcharakteren und Lebensstilen das sozialräumliche Gefüge der gesamten Gesellschaft unverändert; es sind vornehmlich die sozialen Darstellungen in deren mittle- 
rer Region, die fortdauernd in Licht und Farbigkeit changieren. Im Unterschied zu den weitgehend unverändert sich fortsetzenden Modi sozialer Herrschaft der oberen und des Beherrscht-Seins der unteren Klassen reproduzieren sich die mittleren Lagen über ständige Innovationen von Sozialfiguren, Lebensstilen, kulturellen Zusammenschlüssen und Präsentationsformen.

Aus unseren Fallanalysen wollen wir im Folgenden an zwei Fällen die Reisewege in diesem mittleren Terrain nachzeichnen. Wir wählen einen Fall aus der Handballgruppe und kontrastierend dazu einen Fall aus dem Triathlon, der exemplarisch ist für die in dieser Gruppe verbreitete scharfe Ablehnung des traditionellen Vereinssports. ${ }^{6}$ Wir wollen uns gedanklich an den Ort versetzen, den die Befragten im Sozialraum einnehmen, um von dort aus die soziale Logik in ihrem jeweiligen sportlichen Engagement sichtbar zu machen. ${ }^{7}$ Dabei dient uns das Modell des sozialen Raumes als ein sozialwissenschaftlicher Interpretationsrahmen: Indem wir durch dieses Modell hindurch auf unsere Fälle blicken, lassen sich Aspekte sozialen Sinns ans Licht bringen, die einer rein induktiven Fallexegese verborgen bleiben müssen. Zugleich bieten diese Verfahrensweise und die Auswahl der Reisewege die Möglichkeit, das im Vorangegangenen nur ganz allgemein thematisierte Spannungsverhältnis zwischen beharrenden und dynamischen Kräften, das für die in Frage stehende mittlere sozialräumliche Region typisch ist, besser $\mathrm{zu}$ verstehen und genauer $\mathrm{zu}$ kennzeichnen.

\section{Stetigkeit und Beharrung: Die Handballerin Monika}

Monika geht zum Zeitpunkt des Interviews auf die Vierzig zu. Sie ist mit einem Handballspieler und Vereinskollegen verheiratet und hat zwei Kinder. Nach ihrem Abitur absolvierte sie eine Berufsausbildung im Einzelhandel; bis zur Geburt ihres ersten Kindes ging sie diesem Beruf nach. Seitdem ist sie Hausfrau; ihr Mann arbeitet im Gesundheitswesen. Monika spielt seit ihrer Kindheit Handball im selben Verein, in den zu dieser Zeit nicht nur ihre Schwester, sondern auch die Eltern über die Unterstützung des sportlichen Engagements ihrer Töchter eingebunden waren: »Also mein Vater ist jahrelang Sonntag 
morgens aufgestanden und hat uns zum Spiel gefahren oder hat mich gefahren, nach Hause gekommen und dann meine Schwester gefahren.« Mittlerweile sind beide Eltern in Rente. Monikas Vater verfügt über einen Realschulabschluss und eine Meisterurkunde in einem Handwerksberuf. Den Beruf ihrer Mutter, die ebenfalls einen Realschulabschluss gemacht hat, gibt Monika mit >Modistin< an.

Monika ist in einem Bezirk im Westen Berlins, in räumlicher Nähe zu ihrem Handballverein aufgewachsen. Obwohl sie mit ihrer Familie mittlerweile im Süden Berlins wohnt, hält sie ihrem Verein (trotz der nun beträchtlichen Entfernung zum Trainingsort) seit ihrem zehnten Lebensjahr die Treue. Ihre Sportkarriere ist - im Kontrast zu den Fällen aus den anderen Feldern - feindlich gegenüber Experimenten; ihre sportlichen Aktivitäten beschränken sich fast ausschließlich auf das Handballspielen, und sie fallen mit Monikas Vereinszugehörigkeit zusammen.

Bereits zu Anfang des Interviews, wenn Monika schildert, wie sie zum Handball gekommen ist, tauchen die Motive der Stetigkeit und Beharrung auf, durch die ihr sportliches Leben in der Gemeinschaft des Vereins bestimmt ist. Monika findet schon als Grundschülerin zu ihrer Sportart wie zu ihrem Verein und bleibt seither dabei: »Irgendwie hab ich nie daran gedacht aufzuhören und auch nicht daran gedacht, den Verein zu wechseln oder so, weil das war eben dann einfach so.« Als Fixpunkt im Leben, als eine Gemeinschaft, in der man trotz unterschiedlicher individueller Lebenswege bleibt und sich somit überwiegend »von klein auf« kennt, ist der Verein für Monika zugleich eine wichtige Interpretationsfolie, vor deren Hintergrund sie die verschiedenen Abschnitte ihrer bisherigen Lebensgeschichte thematisiert.

Der Fall Monika ist insgesamt durch folgende Besonderheiten gekennzeichnet: Monika kommt aus einer Handwerkerfamilie, einer eher traditionellen Fraktion des Kleinbürgertums. Obzwar in großer vertikaler Nähe zu den Fällen aus den anderen Feldern lässt sich der Fall Monika in Bezug auf ihre Herkunft und ihre Kapitalstruktur (und besonders im Hinblick auf ihr im Vergleich zu den anderen Fällen relativ geringes kulturelles Kapital) in jenem von unseren Fällen insgesamt eingenommenen sozialräumlichen Segment eher im unteren rechten Bereich eintragen. Im Laufe ihres Lebens legt Monika einen 
vergleichsweise kurzen, wenig dynamischen und nur flach ansteigenden sozialräumlichen Reiseweg in Richtung auf den linken (>kulturellen <) Pol des Sozialraumes zurück. Als Protagonistin des Sportvereins verteidigt sie auf exemplarische Weise die >Normalität< des >wahren< in der Vereinsgemeinschaft praktizierten Wettkampfsports gegen alle Erosionen und modischen Tendenzen.

\section{Abgebremste und umgelenkte Aufwärtsmobilltät: Der Triathlet Dirk}

Dirk ist zum Zeitpunkt des Interviews knapp fünfzig Jahre alt, kinderlos und verheiratet. Er entstammt einem kleinbürgerlichen Herkunftsmilieu: Sein Vater und seine Mutter arbeiteten beide als Angestellte im Öffentlichen Dienst. Dirks Vater ist auch im Alter noch (allerdings nicht vereinsgebunden) sportlich aktiv, seine Mutter betreibt keinen Sport. Dirk ist Lehrer und verfügt darüber hinaus über eine Ausbildung in einem kaufmännischen Beruf. Seine Ehefrau arbeitet als Sportlehrerin, war früher aktive Leichtathletin und spielt heute Tennis. Das Ehepaar wohnt in einem Berliner Stadtteil im westlichen Zentrum.

Dirk blickt auf eine fast lebenslange, multidisziplinäre Sportlerkarriere zurück (»ich hab eben irre viel auch schon gemacht«): In seiner Jugend war er Mitglied der Junioren-Nationalmannschaft im Rudern, insgesamt 25 Jahre Mitglied in einem Ruderclub, er hat sechs Jahre lang ambitioniert Fußball gespielt (»unter anderem auch im Olympiastadion, Vorspiel zur Bundesliga«), sich sein Studium, wie er angibt, mit Surf-Unterricht verdient, den ersten Berlin-Marathon mitgemacht, er betreibt seit einiger Zeit auch Volleyball und spielt als Sportlehrer mit seinen Schülern »ein bisschen Basketball«. Dirk hat bereits Mitte der I980er Jahre zum Triathlon gefunden und am ersten in größerem Rahmen veranstalteten Wettkampf in Berlin teilgenommen. Entsprechend selbstbewusst bezeichnet er sich schon bei der ersten Kontaktaufnahme vor dem Interview als »sicher der Beste«, der kompetenteste Interviewpartner $\mathrm{zu}$ diesem Themengebiet und schlüpft in der Interviewsituation in die Rolle des Triathlon-Experten.

Sozialräumlich lässt sich der Fall Dirk folgendermaßen lokalisieren: Dirk entstammt einer eher traditionellen Fraktion des Kleinbür- 
gertums (Vater und Mutter sind kleine Angestellte). Er hat vor diesem Hintergrund einen horizontalen Mobilitätsweg in Richtung auf mehr kulturelles- und Bildungskapital zurückgelegt, nach seiner Ausbildung zum Industriekaufmann ein Hochschulstudium absolviert, um schließlich in eine mittlere Beamtenposition als Lehrer aufzusteigen. Seine horizontale Entfernung von der sozialräumlichen Position seiner Eltern ist ungleich größer als seine vertikale Distanz zu ihnen. Dirk bewegt sich also über seinen Ausbildungsgang, der ihn schließlich zu einer Position als verbeamteter Grundschullehrer führt, aus einer eher stetigen kleinbürgerlichen Region in eine dynamische Teilzone des Sozialraumes.

Diese individuelle Laufbahn erhält durch die Expansion der Bildungsberufe, die wie alle mit hohen kulturellen Kapitalanteilen ausgestatteten neuen Berufe weit überdurchschnittlich zugenommen haben (Vester et al. 200I: 407ff.), gleichsam strukturellen Rückenwind. Dirk profitiert zunächst von der »Öffnung des sozialen Raumes« (Vester et al. 200I: 373ff.), die ihm allerdings - aufgrund nachfolgender Prozesse vertikaler Schließung ${ }^{8}$ - eine in der Perspektive seiner Herkunft lediglich horizontale Positionsveränderung möglich macht. Dirks zunächst strukturell ermutigte Hoffnungen und Ambitionen auf sozialen Aufstieg, auf Distanzierung von seinem Herkunftsmilieu sind horizontal umgelenkt worden, sein Bemühen >nach oben $<$ hat ihn nur in die >linken< Bereiche des sozialen Raumes geführt. Er befindet sich in Bezug auf seine berufliche Stellung als Grundschullehrer zwar im Gravitationszentrum der mittleren Lagen (Vester et al. 200I: 4I6). Vor dem Hintergrund seiner vorwiegend horizontalen Laufbahn, die ihn von seiner Herkunft ein wenig entfernt, vor allem aber in Richtung des kulturellen Pols des sozialen Raumes geführt hat, kann man aber eine gewisse Bewegung weg von den traditionellen kleinbürgerlichen Orientierungen und hin zu den kulturellen Orientierungen eines neuen Kleinbürgertums annehmen. Er nähert sich den modernisierten Fraktionen des Kleinbürgertums an, obwohl hier ein Abstand bestehen bleibt. Im Zuge seiner Akkumulation kulturellen- und Bildungskapitals hat er seinen kleinbürgerlichen Lebensrahmen durch Elemente individueller > Selbstverwirklichung < kontrolliert modernisiert.

Dirks ausgeprägtes Abgrenzungsbedürfnis gegenüber den unteren Schichten (den »dicken«, »niveaulosen«, »proletenhaften« Fußbal- 
lern, »toter Masse« u.Ä.: siehe unten) mischt sich mit einer steten Suche nach dem Neuen, Besonderen und Exklusiven. Wie seine Sportlerkarriere deutlich macht, geht Dirk mit der Zeit und ist dafür auch bereit, mit Traditionen zu brechen: Mitte dreißig gibt Dirk das Rudern und seine langjährige Mitgliedschaft im Ruderclub auf, um mit dem Triathlon zu beginnen, einer zu diesem Zeitpunkt völlig neuen und weithin unbekannten Sportart, die ihm aufgrund ihrer Vielfältigkeit und Intensität als konsequente Fortsetzung und - gegenläufig zu seinem fortgeschrittenen Alter - als Steigerung und Krönung seines bisherigen Sporttreibens erscheint. Im von Dirk gezeichneten Bild des Triathlon dominieren zwei miteinander verbundene Aspekte: Er beschreibt diese Sportart zum einen als eine Form der Intensivierung, Überbietung, ja Eskalation herkömmlichen Sporttreibens und er betont zum anderen ihre gerade mit ihrer Vielseitigkeit verbundene Exklusivität und Besonderheit.

\section{Sog zUR Mitte und Besonderung}

Die sozialräumliche Positionierung unserer beiden Fälle und die vergleichende Darstellung hat den Effekt, die strukturelle Ladung des sportlichen Engagements von Monika und Dirk sichtbar werden zu lassen. Sofort wird deren Unterschiedlichkeit deutlich: Dem sozialen Aufstieg Dirks, der zunächst mit dem Rückenwind sozialer Verschiebungen von einem statischen in ein dynamisches Milieu im mittleren sozialräumlichen Bereich führt, dann aber abgebremst wird, steht das Beharren Monikas gegenüber, die diesem >Wind < eher die Stirn bietet. Die Konfrontation beider Fälle zeigt die Spannungen, die in der turbulenten Mitte des Sozialraumes zwischen inerten und mobilen Klassenfraktionen herrschen. Darüber hinaus wird an beiden Fällen deutlich, dass sich solche Spannungen und Unterschiedlichkeiten gerade mit und durch den Sport besonders gut artikulieren lassen. Monika und Dirk verleihen ihren jeweils spezifischen Bewegungen (bzw. Verharrungen), ihren Positionierungen und ihren damit verbundenen Abgrenzungs- und Unterscheidungsstrategien im sozialen Raum durch ihre charakteristischen sportlichen Praxen eine je spezifische Gestalt:

Monika erzeugt durch ihre sportliche Praxis und ihr Engagement 
im Handballverein ihr geradezu militantes Konzept einer >gesunden<, unbewegten Mitte. Sie schätzt an ihrem Sport dessen kämpferischhemdsärmelige Normalität. Für ihr Bild vom Handball findet sie entsprechend nur wenige, vor allem robuste Formulierungen, die die Abwesenheit feiner Merkmale an ihrem Sport hervorheben; sie artikuliert es vorwiegend ex negativo, durch Kontrastierungen gegenüber anderen Sportarten. So verwirft sie Rhythmische Sportgymnastik und Synchronschwimmen als »sinnlose« formalistische Übungen bzw. als theatralische Darbietungen »mit Band und so 'ne Scherze«, die die Bezeichnung Sport nicht verdienten. Auch von »Einzelsportarten« und »Freizeitbeschäftigungen« wie »Frisbee-Spielen« und »Skateboardfahren« grenzt sie sich ab. Solche Aktivitäten interpretiert sie als Symptome des Verfalls vereinsmäßig organisierten Sporttreibens im Zuge eines gegenwärtig grassierenden Individualismus. Auch schon das Laufen ist ihr in dieser Hinsicht verdächtig: »das ist meistens eine Sache, die man alleine für sich macht und dann irgendwann später nicht mehr im Verein«. Monika profiliert das Handballspielen also nicht nur gegen formalistische, sondern auch gegen individualistische, primär selbstbezogene sportliche Praxen.

Handball steht in Monikas Augen für Substanz statt Form und für Kollektivität und Integration statt Egoismus und Exposition der Person. Im Unterschied zur beredten Thematisierung und Reflexion der sportlichen Praxis in den Selbsterfahrungs-, Lifestyle-, Material- und Technikdiskursen der Triathleten und Inlinehockey-Spieler ist Handball für Monika viel mehr etwas, was man tut und weniger etwas, worüber man spricht. Als eine zu festen Zeiten regelmäßig wiederholte sportliche Praxis formt Handball für Monika in einem ganz körperlichen Sinn immer wieder jene Normalität, die ihr dann als viel zu unspektakulär und selbstverständlich erscheint, um explizit gemacht zu werden. Das wichtigste Ziel, das Monika mit ihrem Sport verbindet, ist »dieses gemeinschaftliche Erleben im Verein«. Dieses Ziel dominiert auch ihren sportlichen Ehrgeiz: »Ich bin nie ganz gut geworden, glaub ich, ich wollt zwar schon gewinnen, aber für mich waren Niederlagen nicht so schlimm und ich hatte also nie diesen absoluten Ehrgeiz, zum Beispiel in 'nen besseren Verein zu wechseln.«

Der Verein erscheint in Monikas Darstellung als eine sich über 
ihre formalen Regelungen und Rahmungen, über die sportliche Praxis und über gemeinsame Unternehmungen selbst erhaltende, ihre Mitglieder bergende wie kontrollierende Institution. Individualismus, Egoismus, Einzelgängertum und letztlich alle außerhalb des Üblichen, des Durchschnitts, der Mitte liegenden, exzentrischen Neigungen und Verhaltensweisen vertragen sich mit dieser Gemeinschaftsform nicht: »Im Verein muss man sich in gewissem Rahmen anpassen!« Das gilt besonders für neu in die Gemeinschaft Eintretende - »wichtig ist, dass ein Neuer am Anfang nicht so die Klappe aufreißt«.

Der Fall Monika ist durch einen generellen Sog zur Mitte gekennzeichnet: Ihrem Streben, innerhalb der Vereinsgemeinschaft in der Mitte zu stehen, entspricht ihre Idealvorstellung vom Verein als einer Sozialform, die durch die verschiedenen biographischen Phasen hindurch ein Haltegerüst bieten und im Mittelpunkt des Lebens stehen sollte. Das von Monika vorgetragene Ethos der Mäßigung und Mitte wird in ihrer Abgrenzung gegen alles Exzentrische, Einseitige und Ungewöhnliche deutlich - vom Einzelgängertum ihres ersten Freundes, das sie mit dessen Familie in Verbindung bringt, die »oft umgezogen ist«, über eine schließlich geschasste Mannschaftskollegin, die »vom Typ her sehr merkwürdig (war), also na ja, Ehe mit einem vierzig Jahre älteren Mann « bis hin zu jenen überspannten höheren Töchtern, die »Konstanze heißen, Klavier spielen und von grünen Bäumen träumen«. In ihrem >weder - noch<, ihrem sich vornehmlich über negative Formulierungen ausdrückenden Selbstverständnis, das sich von allen abgrenzt, die sich abgrenzen, die sich unterscheiden und auffallen wollen, ordnet sie sich selbst einer Fraktion des Kleinbürgertums zu, die in der von ihr eingenommenen, gegenwärtig grundlegend dynamisierten sozialräumlichen Region auf Beharrung und Stetigkeit setzt.

Für Dirk ist Triathlon Intensivierung und Eskalation aller herkömmlichen Formen des Sporttreibens. Triathlon erscheint in seiner Darstellung als ein Ensemble von Körpertechniken, durch die sich die Person vergrößert, aufwertet und unwiderruflich von jeder Gewöhnlichkeit trennt. Die extremen und ständig gesteigerten Belastungen in Training und Wettkampf sind für Dirk Mittel, um zu außergewöhnlichen Erfahrungen zu kommen. Als Triathlet stößt er in einen »Erlebnisbereich vor, der eigentlich, ich glaub, sehr vielen und den meisten 
Menschen einfach verschlossen bleibt«. Die regelmäßig wiederholte sportliche Praxis dient der inneren Akkumulation solch extremer und außergewöhnlicher Erfahrungen. Dirk will sich dadurch $\mathrm{zu}$ einem besonderen Menschen machen, der sich einer elitären, vom Gros der Sportler weit abgehobenen Gemeinschaft von Erfahrenen und Wissenden zurechnen kann.

Die durch Triathlon erzeugte Besonderheit der Person in ihrem Welt- und Selbstverhältnis bleibt dabei keineswegs auf die Innerlichkeit beschränkt. Sie wird durch eine Fülle distinktiver Symbole (Hightech-Bikes, Helme, Sonnenbrillen, Kleidung etc.) dargestellt, nicht zuletzt durch den prätentiösen Duktus, mit dem Dirk beispielsweise im Umziehen nach dem Schwimmen und vor dem Fahrradfahren eine »Faszination des Wechsels« ausmacht. »Die Triathleten sind einfach ein buntes Volk, was nach außen hin auch auf das Outfit achtet, also sei es jetzt Klamotten, sei es Fahrrad, sei es Körperbetonung. [...] Also da sind die Triathleten viel extrovertierter also nach außen und finden Medien gut und dies, und das ist eine tolle Sache eigentlich.« Die Distinktion der »eher akademischen« Sportart Triathlon gegenüber populäreren sportlichen Betätigungen wie Fußball - Dirk zufolge »die Sportart des Proletariers« - drückt sich für ihn ganz entscheidend in den jeweiligen Körperformen der Akteure aus. Im Unterschied zu den häufig »dicken Fußballern« ist der Triathlet stets bemüht, »gut austrainiert zu sein, also eben wirklich wenig tote Masse mit sich rumzuschleppen [...], das heißt also, die Körperproportionen sind zu sehen, die Muskulatur ist zu sehen und nicht verdeckt durch tote Masse . Der Ausdruck »tote Masse« kennzeichnet nicht nur die unteren Klassen. Er steht zugleich für das Träge, Schwere und Inerte, für den >Proletarier in ihm selbst<, gegen den Dirk unaufhörlich Krieg führt. Man kann »tote Masse« also auch als körperliche Metapher für Dirks festgezurrte soziale Identität als Grundschullehrer lesen; eine soziale Identität, aus der er - nachdem sein zunächst dynamischer sozialräumlicher Reiseweg zum Stillstand gekommen ist - nun durch Triathlon, d.h. durch auf sich selbst gerichtete übersteigerte Anstrengungen, auszubrechen trachtet. 


\section{ZWEI Konträre SelbStVerhältinisse}

Mit dem in Vereinsmannschaften gespielten Handball und dem individualistisch betriebenen Triathlon stehen sich zwei deutlich voneinander unterschiedene Ausprägungen des Sporttreibens gegenüber. Nicht nur die Bewegungsweisen kontrastieren, sondern auch die Trainings- und Wettkampforte, die Gemeinschaften, Rituale, Codes und Kleidungsstile sind im neuen Spiel deutlich anders als im traditionellen Handball. Was von den Triathleten an ihrem Sport positiv bewertet wird: individuelle Sportausübung, geringe Bedeutung der Gemeinschaft und die Abwesenheit von Bindungen, Ästhetisierung und Spektakularisierung von Körper und Bewegung, öffentlich präsentierter Narzissmus, wird aus der Perspektive der traditionellen Sportlerinnen ausdrücklich negativ beurteilt. Für sie ist gerade die institutionell garantierte Beständigkeit des Vereinslebens wichtig. Alle diese Unterschiede kommen in der Art und Weise zusammen, wie die Akteure sich selbst auffassen: in ihrem Bild und Entwurf von sich selbst und in ihrem Verhältnis zu ihrer sozialen Herkunft und Klassenzugehörigkeit. Die Angehörigen beider Sportgruppen sind, obwohl sie sich sozialräumlich sehr nahe stehen, dennoch durch deutlich unterschiedene Habitus gekennzeichnet.

Eine Reihe von Indizien sprechen dafür, dass die Triathleten den von uns abkürzend als >kleinbürgerlich< bezeichneten Habitus des kleinen und mittleren Bürgertums, der bei den Handballerinnen deutlich hervortritt, $\mathrm{zu}$ neuen Ausprägungen weiter entwickelt haben. Wenn dies zutrifft, dann stellt sich die Frage: Wie lässt sich eine solche Habitusveränderung im Rahmen der Bourdieuschen Soziologie theoretisch darstellen? Dass sich in unmittelbarer Nachbarschaft im sozialen Raum so unterschiedliche Spielvorlieben wie die für den traditionelle Handball und jene für die extreme Sportart Triathlon ausprägen, scheint auf den ersten Blick mit der Annahme eines durch den Habitus geordneten sozialen Handelns unvereinbar zu sein. Es lässt sich aber zeigen, dass der Habitusbegriff angesichts eines solchen scheinbaren Durcheinanders nicht überfordert, sondern gut anwendbar ist, vorausgesetzt, man erfasst seine Dynamik. ${ }^{9}$

Ein Habitus ist niemals statisch; er kann freilich gegenüber einer sich wandelnden sozialen Umgebung den Eindruck von Unbeweg- 
lichkeit machen. Zu völliger Immobilität gelangt er nie, insofern er die Veränderungen, Schwankungen seiner Umgebung zumindest ausgleicht. Die Handballerinnen in unserer Untersuchung kann man bei eben solchen Versuchen beobachten. Ihr Habitus ist beharrend - in ihrem Verein wird Sport getrieben wie zu Zeiten ihrer Eltern, in ihrem Stadtviertel, zu festen Trainingszeiten, der Mannschaftsdisziplin unterworfen, den Anordnungen des Trainers gehorchend, mit gemeinschaftlichen Kneipentreffen nach dem Training und Punktspielen am Wochenende. Sie entwickeln keine neuen Verhaltensweisen, sondern beharren auf geerbten Werten, Idealen und Lebensweisen. Dies geschieht nicht als ein verbissener Kampf, sondern aus dem Vergnügen an der Sportpraxis, am harten agonalen Körpereinsatz und am Vereinsleben mit seinen Freundschaftsbeziehungen. Die Art und Weise, wie die Handballerinnen ihre Sportpraxis unter gänzlich veränderten Umweltbedingungen fortsetzen, zeigt die Belastbarkeit ihres Habitus. Gerade der traditionelle kleinbürgerliche Habitus besitzt eine beharrliche Stabilität; er reproduziert Bekanntes und gibt Sicherheit gegenüber Veränderungen.

Aber um die Handballerinnen herum ist die Zeit nicht stehen geblieben. Im Unterschied $\mathrm{zu}$ ihren Vorgängern müssen sie sich gegen Fitnessclubs und neue Sportarten zur Wehr setzen, die ihnen zuwider sind, gegen die Erosion, die das Vereinsleben zersetzt, die Egoismen, die veränderten Prioritäten und Werte der jüngeren Mannschaftsmitglieder. Gegenüber allen diesen Veränderungen ist die Vereinswelt des Handballs eine Insel geworden, auf der man etwas findet, was inzwischen Seltenheitswert hat. Die Mitglieder können sich nicht mehr, wie noch ihre Eltern und Großeltern, als eine Avantgarde des sportiven Lebensstils empfinden. In ihrer unmittelbaren Nachbarschaft im sozialen Raum werden neue Entwürfe ausprobiert, neue Weisen der Sportausübung und der Gemeinschaftsbildung erprobt, die sich an Kleidung, Bewegungsformen, Gestiken, motorischen und materiellen Techniken zeigen.

Neben der Beharrung sind aber auch Aufstieg, Ehrgeiz und Verunsicherung wichtige Merkmale des kleinen und mittleren Bürgertums. Hier geht es um Verbesserung der Lebensverhältnisse, um das Aufschließen zu höher gestellten sozialen Gruppen, nicht nur in Beruf, Einkommen, sozialer Stellung, sondern auch in Lebensstil und 
-gewohnheiten, um die Aneignung neuer Praktiken, was den aufgestiegenen Kleinbürgern immer wieder Spott über ihre parvenühaften Allüren einträgt. Das Abrücken von traditionellen Positionen, die flinke Bereitschaft, die Herkunft vergessen zu machen, können als Stärke und Schwäche dieser sozialen Klassenfraktion zugleich gelten. In ihren eigenen Augen ist diese Haltung insofern eine Quelle von Dynamik, als sie sich ihren Aufstieg als persönliches Verdienst, als Resultat ihrer eigenen Tüchtigkeit zurechnen, selbst wenn nicht sie ihres Glückes Schmied sind, sondern wenn sie von Zeitumständen, wie der Bildungsexpansion, begünstigt wurden. Der Glauben, die eigene soziale Aufwärtsbewegung ausschließlich sich selbst zu verdanken und ein Unternehmer in eigener Sache zu sein, macht die schiefe Sicht dieser Gruppe auf soziale Prozesse aus. Gegenwärtig kleidet sich diese Angestelltenideologie in Ausdrücke wie »Arbeitskraftunternehmer« oder »Ich-AG «.

Was aber die Einstellung des Triathleten Dirk von dieser traditionellen Selbstdeutung des Kleinbürgertums unterscheidet, ist die Tatsache, dass er nicht eine soziale Höherbewegung nachträglich rationalisiert. Einen gewissen kleinen Aufstieg hat er zwar tatsächlich erreicht, aber er befindet sich jetzt in der Phase danach, wo dieser zum Stillstand gekommen ist und ein weiteres Steigen nicht mehr erwartet werden kann. Seine Dynamik und seine Selbstdeutungen entfaltet er in einem neuen Betätigungsfeld, in der Freizeit. Sie werden nicht im Rückblick wirksam, sondern richten sich auf die Verwirklichung eines neuen Entwurfs von sich selbst in der Zukunft. Der Selbstentwurf ist ein Modellieren der eigenen Person im Sport.

In den beiden unterschiedlichen Untersuchungsgruppen stehen sich zwei konträre Ausprägungen des kleinen und mittleren Bürgertums gegenüber. Auch die Handballerinnen stellen ihr Selbstverhältnis dar; in den Interviews geben sie eine Fülle von sie selber interpretierenden Kennzeichnungen. Sie werden nicht müde zu sagen, wer sie sind und wie sie sind. Im Unterschied zur Gruppe der Triathleten führen sie einen vorgefundenen Entwurf fort. In dem Augenblick, in dem sie sich im Sportverein engagieren, steht fest, dass sie die vorgefundenen Elemente übernehmen. Ihr Sportengagement mit allen seinen Besonderheiten, die vereinsfernen Personen absonderlich vorkommen, wird auf dieser Bahn zu einer Selbstverständlichkeit, die 
keiner Rechtfertigung bedarf. Die Vereinspraxis erzeugt ihren Sinn, ihre Handlungsweisen, Werte, Anerkennung aus sich selbst. Sport ist hier eine Aktivität der persönlichen Bewährung durch Leistung und Verlässlichkeit, in einem erworbenen, festen Habitus verankert.

Auf solche Sicherheiten greifen die Triathleten gerade nicht zurück; sie sind ihnen suspekt. Ihnen geht es um einen neuen Entwurf von sich selber. Dieser unterscheidet sie von dem Ich, das sie im Berufsleben, in den Bereichen außerhalb des Spiels verwirklichen; in ihrer Sportpraxis werden sie zu einem anderen. Sie haben es auf eine performative Umgestaltung ihres Ichs im Spiel abgesehen. Performativ ist ihre Praxis, weil sie das >neue Ich ` vorführen, es anderen in actu zeigen. Die entworfene Person ist kein Ausdruck irgendeines TiefenIchs, sondern wird in sportlichen Aktivitäten erst entfaltet. Dass die Neukonzeption im Spiel stattfindet, macht es gewiss leichter durchsetzbar, weniger folgenreich, aber nicht unernster als ein vergleichbarer Versuch im Berufsleben (wo er überall an Grenzen stoßen würde). Triathlon verlangt ein außerordentlich tiefes Engagement, große Härte und Unerbittlichkeit gegen sich selbst, ein rigides Zeitmanagement. Diese Anforderungen greifen weitgehend in das Leben der Athleten ein. Es ist nicht übertrieben $\mathrm{zu}$ sagen, dass im Sport eine andere Existenzweise ausgelebt wird als im Berufsleben. Im Bereich der Spiele geben die Athleten mit aller Konsequenz ihrem Leben einen Stil.

Die von Foucault vorgebrachte, auf Nietzsche zurückgehende Forderung, man solle seinem Leben einen Stil geben, ${ }^{\text {IO }}$ wird hier, im Bereich des Unernsten, verwirklicht. Während das Alltagsleben der Triathleten, insbesondere im Vergleich $\mathrm{zu}$ ihrem extremen Sport, durch Abwesenheit von einem selbstgeprägten Stil gekennzeichnet ist, findet man hier den Willen zu einem neuen Stil. Die große Anstrengung des Durchhaltens und der Leistungsverbesserung kann als Antwort auf die Frage verstanden werden, die sich die Athleten mit größtem Ernst stellen: Bin ich fähig, meinen Ich-Entwurf zu verwirklichen? Sport ist hier eine Arbeit an einem neuen Ich. Der Athlet entwickelt ein neues Selbstverhältnis, indem er sich selber gegenüber als Fordernder auftritt und sich von sich selbst eine neue Haltung verlangt. Er verlangt von sich Treue zum Stil. 


\section{Die Veränderung des Habitus aus der Entstehung eines neuen Selbstverhältinisses}

Das Selbstverhältnis, ein Begriff, den wir von Foucault übernommen haben (vgl. Gebauer 2003), lässt sich als ein Bestandteil des Habitus deuten, insofern zu diesem auch die Fähigkeit gehört, zu sich selbst Stellung zu nehmen und sich Gewissheit darüber zu verschaffen, wer man ist und wie man ist. ${ }^{\text {II }}$ Gegenüber der traditionellen geistphilosophischen Deutung des Selbst hat Bourdieus Habitusbegriff (und Foucaults Konzept des Selbstverhältnisses, das man als integrativen Teil des Habitus auffassen kann) einen großen Vorteil: Man muss keine Tätigkeit des Selbstbewusstseins und keine Selbstbeobachtung des Geistes annehmen, wenn man beschreiben will, wie der Handelnde die Fragen nach dem Wer und Wie seines Selbst beantwortet. Bourdieus Entdeckung ist gerade, dass wir im Handeln Strukturen realisieren, die körperlich in uns eingegangen sind und die wir wie selbstverständlich, ohne Reflexion vollziehen, die unsere Handlungen sind, uns zugehörig sind, die wir verwirklichen und wollen. Wer und wie wir sind, ist uns nicht ein für allemal gegeben - dies stellen wir in unserer Handlungspraxis immer wieder von neuem her, und zwar auf eine solche Weise, dass wir eine Folge von Handlungen hervorbringen, Entscheidungen treffen, Wertungen fällen, die sich alle auf irgendeine Weise ähnlich sind.

Das Merkmal, das diesen Handlungen gemeinsam ist, kann man nach Wittgenstein Familienähnlichkeit nennen (vgl. Wittgenstein I993). Damit wird ausgedrückt, dass es nicht ein ihnen allen zugrunde liegendes, essenzielles Kennzeichen ist, sondern dass von Handlung zu Handlung eine fortlaufende Ähnlichkeitsbeziehung hergestellt wird, die in ihrem Fortlaufen die Differenzen der Ähnlichkeit ständig verändert, sodass alle Glieder der Kette allen anderen ähnlich sind, aber nicht in einem, sondern in unzähligen und unterschiedlichen Merkmalen. In diesem Sinne kann man davon sprechen, dass das Subjekt in seiner alltäglichen Handlungspraxis Selbst-Ähnlichkeit herstellt; und dies gerade nicht aufgrund von Nachdenken und durch Selbstbewusstsein gesteuerte Entscheidung.

An der Herstellung unserer Selbst-Ähnlichkeit ist wesentlich der 
Geschmack beteiligt. Der Geschmack ist das Organ des sozialen Urteilens; diesen Gedanken hat Pierre Bourdieu von Kant übernommen und konsequent soziologisch umgedeutet. Der Geschmack beurteilt das sinnlich Gegebene. Diese Art des Urteilens ist nun keine reine Verstandestätigkeit, insofern es in Praxis getaucht ist. Wer wir sind und wie wir sind, erkennen wir selber und die anderen, die uns zusehen oder gemeinsam mit uns handeln, insbesondere an den Produktionen unseres Geschmacks: daran, wie wir auftreten, wohnen, gekleidet sind, an der Sorge um den Körper, an der Wahl unserer Freunde, Partner, unserer Freizeitbeschäftigung, künstlerischen Vorlieben und an dem Sport, den wir ausüben.

Zum Prinzip der Selbst-Ähnlichkeit aufgrund des Habitus gehört, dass man in den verschiedenen sozialen Feldern strukturell homolog handelt. So kommt es, dass man im Feld des Sports seinen erworbenen Habitus unter den besonderen Bedingungen dieses Feldes verwirklicht. Man setzt im Sport also den Habitus, den man in seiner Lebensgeschichte ausgeprägt hat, auf spezifische Weise fort. Dies genau tun die Handballerinnen: sie bekräftigen ihren erworbenen Habitus im Feld des Sports. Sie handeln hier so, dass jede von ihnen sich durch ihre Sportausübung, ihre Redeweise, ihre Urteile als ein spezifisches Ich kennzeichnet. Sie üben Treue zu ihrer sozialen Position und verlangen auch von den Mannschaftskameradinnen, dass sie unter allen Umständen sich selbst ähnlich bleiben sollen: Bloß nicht anders werden, nicht komisch werden! Die Triathleten wollen das genaue Gegenteil mit ihrem Wunsch, einen neuen Selbstentwurf zu leben. Ihr Ziel ist gerade die Selbst-Unähnlichkeit.

Man kann jetzt deutlich erkennen, dass die Triathleten mit ihrer Absicht, das Selbstverhältnis neu zu formen, in letzter Hinsicht auf eine Veränderung ihres Habitus zielen. Mit ihrer Entscheidung gegen den hergebrachten Habitus verwirklichen sie eine in diesem selbst angelegte Disposition. Der Wunsch nach Existenzveränderung kann selbst als Bestandteil ihres Klassenhabitus aufgefasst werden. In den neuen Sportpraktiken treffen Akteure mit Dispositionen zur Habitusveränderung, die für bestimmte Fraktionen des >Kleinbürgertums kennzeichnend sind, auf ein strukturelles Angebot, welches ihnen erlaubt, diese Veränderungswünsche auszuleben und dabei soziale 
Anerkennung zu erhalten. Genau hier tritt aber ein Unterschied zu Bourdieus Habituskonzeption auf: eine Öffnung, die das Emergieren von Neuem ermöglicht.

Der Ausgangspunkt von Habitusveränderung ist in unserem Beispiel die Tatsache, dass der Geschmack der Triathleten anders funktioniert, als er dies bei der Produktion und Bewertung von Sportpraktiken üblicherweise tut. Wenn er seine Selbstverständlichkeit, sein reibungsloses Funktionieren, die Sicherheit seines Einsatzes verliert, löst sich auch die feste Verbindung zwischen Sinnlichkeit und Bewertung. Die selbstverständliche Beziehung zwischen der Wahrnehmung von Praktiken und ihrer Deutung erhält dann einen Sprung. Wenn also der Geschmack, den man in seiner Lebensgeschichte erworben hat, nicht mehr wie üblich funktioniert, entsteht ein Abstand zur üblichen Wahrnehmung und Bewertung der Erfahrungswelt.

Eine solche Situation, in der die Verbindung von Sinnlichkeit und Geschmacksurteil ihre Festigkeit verliert und gleichsam flüssig wird, ist ein ungewöhnlicher Zustand. Üblicherweise ist es der Geschmack, der, wenn er sich in den Lebensverhältnissen des Subjekts gebildet hat, dessen sozialem Handeln Halt und Festigkeit gibt. Seine Wirkungsweise setzt schon früher an als beim Hervorbringen von Handlungen und Urteilen, insofern er die sinnlichen Wahrnehmungen der Subjekte formt. Bourdieu bezeichnet den Geschmack als »den praktischen Operator für die Umwandlung der Dinge in distinkte und distinktive Zeichen, der kontinuierlichen Verteilungen in diskontinuierliche Gegensätze« (Bourdieu I982: 284). Was in der sinnlichen Welt zusammenhängend und körperlich da ist, wird zu Gegensätzen angeordnet, sodass aus physischen Erscheinungen Zeichen entstehen. Der Geschmack überführt »die Unterscheidungen, die in die physische Ordnung der Körper eingeschrieben sind, in die symbolische Ordnung der sozialen Distinktionen « (Bourdieu I982: 284). Durch diese Operation erhalten sie einen zeichenhaften Charakter, insofern sie gesellschaftliche Unterschiede bezeichnen und klassifizieren. Der Geschmack ist der Urheber des Systems sozial unterscheidender Merkmale, mit denen eine soziale Klasse gekennzeichnet wird. Diese bilden einen klar erkennbaren »Lebensstil«, der auf den »konstitutiven >Entscheidungen« « des sozialen Geschmacks beruht (Bourdieu I982: 285).

Der als Operator wirkende Geschmack stellt Verknüpfungen 
zwischen Sinnlichkeit und sozialen Bedeutungen, Bewertungen und Positionen her, die durch die soziale Erfahrung zu festen Verbindungen gemacht werden, insofern die sinnlichen Wahrnehmungen, Reaktionen, Wahlen, Beurteilungen weitgehend sozial festgelegt sind. Aber dies heißt nicht, dass sie determiniert sind; es können neue Sinneseindrücke auftauchen, die nicht automatisch mit sozialen Bedeutungen und Bewertungen versehen werden. In solchen Situationen entsteht etwas Neues - neue Züge des Habitus oder Züge eines neuen Habitus.

Betrachten wir die von Bourdieu skizzierte Struktur genauer: Differenzen der körperlich-sinnlichen Ordnung werden in die symbolische Ordnung der Distinktionen überführt. Dies geschieht nicht von selbst, sondern ist eine Leistung des Geschmacks; er ist zugleich Urheber der symbolischen Ordnung. Seine Wirkungen entfaltet er unablässig im sozialen Handeln, überall da, wo wir körperlich-sinnlich handeln, entscheiden und urteilen. Diese Prozesse vollziehen sich hinter dem Rücken der Akteure - weil sie im praktischen Handeln unerkannt bleiben, können sie distinktiv sein; und weil der Geschmack nicht reflektiert wird, gibt er Sicherheit im Wahrnehmen, Handeln und Urteilen.

Wir denken nicht nach, wenn wir geschmacklich wählen oder urteilen; wir reagieren reflexhaft in Geschmacksdingen, insofern wir uns den körperlichen Gefühlen des Gefallens und Missfallens überlassen. Bestimmte Dinge erfüllen uns mit Abscheu, erregen geradezu physische Übelkeit, andere verschaffen uns Vergnügen. Der Geschmack gibt uns die Fähigkeit, sinnlich-körperlich auf die Welt zu reagieren und uns mit diesen Reaktionen jener Position im sozialen Raum anzupassen, die unserem Geschmack entsprechen und die wiederum durch diesen bestimmt werden. Was wir aufgrund unseres Geschmacks tun, gibt Auskunft über uns, sagt uns und den anderen, wer wir sind und wie wir sind. »Der Geschmackssinn ist ein Sinn, in dem man sich gewissermaßen selbst sinnlich wahrnimmt (Arendt I998: 92).

Wirkt der Geschmack notwendig immer automatisch, wie Bourdieu annimmt? Gibt es nicht auch Gelegenheiten, bei denen er seine aus dem Habitus stammende >Automatik suspendieren kann? Als Ausgangspunkt einer möglichen Veränderung des Habitus kann man 
die Situation auffassen, wo das handelnde Subjekt sein Selbstverhältnis zu verändern sucht - in diesem Moment nimmt es Abstand zu seinem Geschmack. Es suspendiert vorübergehend dessen habituelles Funktionieren, Urteilen und erzeugt sich einen bestimmten Spielraum. Die übliche automatisierte Geschmacksproduktion wird unterbrochen; die ansonsten kontinuierlich ablaufende Überführung von körperlich-sinnlichen Merkmalen in Distinktionen findet jetzt nicht mehr statt. Wenn das Subjekt nun - sei es auch nur im Spiel, für eine gewisse Zeit - sein Selbstverhältnis neu entwirft, sein Ich zum Gegenstand von Reflexion macht und dieses schließlich umbaut, verändert es auch partiell seinen Habitus.

Aus der Tätigkeit des Geschmacks bilden sich Züge eines veränderten Habitus in solchen Handlungsbereichen, in denen es keine >starren Verbindungen zwischen körperlich-sinnlichen Erscheinungen und der sozialen symbolischen Ordnung gibt. Insofern hier keine Automatismen der Kategorisierung und Interpretation am Werke sind, kann hier, mit Hilfe praktischer Reflexivität, ein freies Spiel der Vorstellungskräfte möglich werden. Im Feld des Sports, der als ein »l'art pour l'art des Leibes« (Bourdieu ı986: 95) verstanden werden kann, ist ein solcher Spielraum unter bestimmten Umständen möglich: wo die Sinneseindrücke und körperlichen Erfahrungen auf die Beschaffenheit des Subjekt selbst gerichtet sind, als eine frei flottierende Erfahrung.

Aus unseren Interviews mit Teilnehmern an den neuen Spielen spricht die Lust, sich einen Freiraum zu schaffen, Abstand vom hergebrachten Geschmack und Selbstverhältnis, vom Automatismus der sozialen Bewertungen zu gewinnen. Dies geschieht zwar spielerisch, in einem Bereich, dem üblicherweise Beliebigkeit zugeschrieben wird. In der Bourdieuschen Soziologie sind jedoch Veränderungen des Habitus in einem sozialen Feld, und sei es auch in dem der Spiele, weder beliebig noch total folgenlos für die anderen sozialen Felder. Der Ernst, mit dem Triathlon betrieben wird, sein tiefes Eingreifen in Lebensvollzüge außerhalb des Sports, lässt eine allmähliche Sedimentierung der Habitusveränderungen, die hier entstehen, erwarten.

Sozialer Wandel, der in einem sozialen Feld eintritt, verändert andere Felder nicht nur in ihrem speziellen Funktionieren, sondern auch strukturell. Wenn man im Spiel mit aller Ernsthaftigkeit einen 
anderen Entwurf von sich selbst lebt, wird dieser nicht einfach mit einem direkten Transfer in ein anderes Feld transportiert, aber es kommt zu Veränderungen in der Struktur des Habitus. Es ist also nicht zu erwarten, dass der Triathlet das neu entworfene Ich, das er in seinem Sport konsequent, glaubwürdig durchgesetzt hat, in seinem Arbeitsleben ausspielt - er wäre lächerlich und unglaubwürdig. Aber im Habitus des Subjekts ist von der Sportaktivität eine strukturelle Latenz angelegt worden: eine Bereitschaft, zum erworbenen Selbstverhältnis Distanz einzunehmen; die Möglichkeit, sich als anders zu deuten; die Fähigkeit, die körperlich-sinnlichen Eindrücke anders als reflexhaft in die symbolische Ordnung zu überführen. Das Subjekt hat sich neue Sichtweisen, Verhaltensmöglichkeiten, Handlungschancen gesichert, die nicht mehr den alten Urteilsweisen vorgelegt werden müssen, sondern auf eine neue Weise bewertet werden können.

Neue Spiele schaffen neue Fakten, aber noch keine klar erkennbaren, ausgeformten und reproduzierbaren Sachverhalte. Ihre Hervorbringungen sind noch scheue Neuheiten. Das Neue hat erst dann eine Chance, zu feldübergreifenden Veränderungen zu führen, wenn sich auch in der Gesellschaft eine strukturelle Entsprechung findet. Es lässt sich annehmen, dass auch in den Strukturen, in denen sich das kleine und mittlere Bürgertum organisiert, die Tendenz auftritt, den herkömmlichen Habitus zu distanzieren, alte Sicherheiten loszulassen, sich zu riskieren und neue Praktiken zu suchen. Im Zusammenhang mit makrostrukturellen Veränderungen ist eine Veränderung des Geschmacks eingetreten, die sich in andersartiger Kleidung, Essensgewohnheiten etc. bei weitem nicht erschöpft, sondern Körperlichkeit und Sinnlichkeit anders als bisher wahrnimmt und bewertet. Veränderungen des Ich-Entwurfs setzen an Körper und Sinnlichkeit an, zum einen in Vorstellungen und Handlungen der Subjekte, zum anderen in den Möglichkeiten, die die Gesellschaft zur Verfügung stellt, indem sie den Freiheitsspielraum erweitert, Modelle anbietet, neue Bewertungen und Normen zulässt. Eine solche komplexe, die Subjekte anreizende Struktur ist nicht weniger als eine Aufforderung an die Subjekte: Euer Ich sei ein anderes! Die Botschaft des KünstlerSubjekts des I9. Jahrhunderts ist im Kleinbürgertum der Gegenwart angekommen. 


\section{SPORT als SOZIALE REPRÄSENTATIONSARBEIT}

Wir haben diese Tendenz zur Habitusänderung bisher in Bezug auf den einzelnen Akteur dargestellt. In der Bourdieuschen Soziologie haben subjektive Vorgänge dieser Art immer ein objektives Gegenstück in den Strukturen der jeweiligen Klassenfraktion. Die darin zum Ausdruck kommenden Entwurfswünsche und Tendenzen zur Überschreitung und Neufassung des Ichs sind keine isolierten Einzelerscheinungen, das Subjekt teilt sie mit anderen, bei denen sich gleichartige, allmählich nach außen dringende Tendenzen bemerkbar machen. Der Wunsch nach Veränderung des Ichs als charakteristische Tendenz einer Fraktion des kleinen und mittleren Bürgertums ist ein von vielen Mitgliedern derselben sozialen Fraktion geteiltes Verlangen. Offensichtlich leisten Sportpraktiken einen Beitrag für die Herausbildung lebensweltlich formierter und sichtbarer Fraktionen im mittleren Bereich des sozialen Raumes. Sie machen nicht nur das Entstehen neuer Gruppierungen erkennbar, sondern geben den einzelnen Fraktionen des Kleinbürgertums überhaupt die Möglichkeit der Wiedererkennbarkeit. Noch einmal wenden wir uns den beiden Fallanalysen zu, um deren jeweilige Selbst- und Fremdklassifizierungen genauer zu betrachten.

Monika entwirft ihr Bild vom Handball überwiegend durch Abgrenzungen nicht nur gegenüber anderen Sportarten, sondern auch gegenüber deren Akteuren. So kann man in den von der robusten Handballerin abgelehnten Gymnastinnen und Synchronschwimmerinnen, die sich in ihrer Perspektive sinnlosen formalistischen Übungen widmen, unschwer Sozialfiguren erkennen, die ein anderes soziales Milieu repräsentieren. Formvollendete Ausführungen von zweckfreien Körperbewegungen erscheinen ihr künstlich; ihnen mangelt es an jenem Realismus, der die Körpereinsätze im Handball (»Körperkontakt haben, Laufen und Springen«) kennzeichnet, die »aus dem Leben kommen«. Monikas Ablehnung der Form zugunsten der Funktion folgt einem traditionell kleinbürgerlichen Muster der Kritik an formalen Verhaltens- und Gestaltungsweisen. In ihrer Kritik der formalistischen Sportpraxen von Gymnastinnen und Synchronschwimmerinnen weist sie die Vertreterinnen eines Milieus zurück, dessen 
ästhetische Einstellungen und kulturelle Orientierungen sie zutiefst ablehnt und von dem sie sich strikt abgrenzt.

Auf der anderen Seite klassifiziert Dirk die Triathleten als eine exklusive Gemeinschaft von Individualisten, die sich über ihr Outfit und ihr Equipment nach außen als stilistisch progressive Modernisten präsentieren und sich dadurch vom traditionellen Bild des Vereinssportlers unterscheiden. Für diese Selbstklassifizierung bemüht er wiederholt das Negativbild der »proletenhaften Fußballer«: »Wenn ich das vergleiche, Triathlon mit Fußball, dann fällt der Fußball ja extrem ab, weil da herrscht eine Ausdrucksweise, ein Niveau unter den Leuten, das also mit dem Triathlon [...] überhaupt nichts zu tun hat.« Die Abgrenzung gegenüber einer anderen Sportart ist zugleich eine soziale Grenzziehung, die dem eigenen wie dem abgelehnten Milieu austrainierten und gestählten Individualisten wie grobschlächtigen, ignoranten Fußballern - körperliche Gestalt verleiht. Die Selbst- und Fremdzuordnungen, die jeweils breiten Raum einnehmen, wenn Monika und Dirk über ihren Sport sprechen, artikulieren in beiden Fällen mit körperlich demonstrierter Evidenz soziale Unterscheidungen. Über die jeweilige Sportart, von der die Rede ist, vollzieht sich eine Veranschaulichung und eine körperliche Verwirklichung unterschiedlicher sozialer Gruppen.

Man kann das Engagement im Handballverein wie in der Triathlon-Gemeinschaft in dieser Hinsicht als eine je spezifische Form der »Repräsentationsarbeit« (Bourdieu I985: I6) verstehen, durch die bestimmte Akteursgruppen versuchen, »ihre gesellschaftliche Identität durchzusetzen« (Bourdieu I985: I6). Dabei wird das Repräsentierte in dieser Darstellungsaktivität überhaupt erst erzeugt. ${ }^{\mathrm{I2}}$ In einer solchen sportlichen Repräsentationsarbeit geht es ganz wesentlich um ein »Sichtbarmachen des Andersseins« (Krais I990: 342). So erhalten die unterschiedlichsten Fraktionen des Kleinbürgertums in den körperlichen, insbesondere sportlichen Aufführungen eine Wirklichkeit, die sie als moderne, dynamische oder traditionelle, beharrende Gruppen erkennbar macht. Auf diese Weise beginnen die Gruppen von Akteuren mit ähnlichen sozialen Existenzbedingungen als sichtbare soziale Formationen zu existieren. ${ }^{\mathrm{I}}$

Die Erzeugung einer sozialen Klassenfraktion durch sportliche 
Repräsentationsarbeit lässt sich gut am Beispiel des traditionellen Kleinbürgertums deutlich machen, von dem man ohne Verweise auf Schrebergärten und nicht zuletzt den Sportverein gar nicht mit lebensweltlicher Anschaulichkeit reden könnte. ${ }^{\mathrm{I}}$ Charakterisierungen typisch kleinbürgerlicher Sozialfiguren und Milieus knüpfen häufig an deren durch die Vereine erzeugte soziale Sichtbarkeit an. ${ }^{15}$ Die besondere und bis in die Gegenwart stabile Verbindung zwischen dem Sport und den Mittelklassen entsteht schon im Laufe des >langen I9. Jahrhunderts<. Die bürgerlichen Mittelschichten dieser Zeit entdeckten und gebrauchten die aus England importierten >sports< als eine Möglichkeit, um - im Angesicht gesellschaftlicher Instabilitäten »Bürgerlichkeit aus sich selbst heraus zu erzeugen« (Eisenberg I999: 43I).

In der Gegenwartsgesellschaft haben sich offenbar - wie man im Hinblick auf die Veränderungen und Neuerungen im Bereich des Sports schließen kann - die Formen von Bürgerlichkeit und die Modi ihrer Erzeugung durch den Sport gravierend verändert und vervielfältigt. Im Unterschied zum traditionellen Vereinssport mit seinen festen Orten und Zeiten repräsentieren sich neue sportliche Gemeinschaften wie die der Triathleten oder die der Inlinehockey-Spieler nicht länger nur zu bestimmten Anlässen oder an speziell dafür eingerichteten Orten. Indem die Akteure des postkonventionellen Sports ihre sportliche Praxis zeitlich und räumlich universalisieren, schaffen sie eine neue Einheit von sportlicher Repräsentation und alltäglichem Leben: die Sporthallen und Sportplätze werden verlassen, die besonderen Anlässe vervielfältigt, die Bühnen werden verallgemeinert und die Hinterbühnen abgeschafft, die Akteure steigern ihre soziale Sichtbarkeit und reklamieren für sich eine Schlüsselstellung in den Prozessen der Gesellschaftsveränderung. Das neue Kleinbürgertum wird extrem - im Sport.

\section{Anmerkungen}

I Der spezifischen Anlage und Erkenntnisstrategie unserer empirischen Untersuchung folgend, wird hier auf quantifizierende Aussagen verzichtet, denn die Auswahl der Fälle im jeweiligen 
Untersuchungsfeld orientierte sich an deren Typik und nicht an ihrer statistischen Repräsentativität für das Feld. Diese Typik der Fälle für die Felder haben wir methodisch durch die Kontrastierung verschiedener Fälle innerhalb der Felder und über die verschiedenen Felder hinweg untersucht. Die folgende Aufzählung verschiedener sozialer Positionen will entsprechend nur für alle drei Felder typische (keinesfalls aber die häufigsten) Bildungsstände und Berufstätigkeiten nennen.

2 Die Gruppe der Inlinehockey-Spieler hat ein im Vergleich zu den anderen beiden Sportgruppen relativ geringeres Kapitalvolumen, da die Akteure jünger sind und sich mehrheitlich noch in Ausbildungsphasen befinden. In unserer Grafik (Abb. I) wird dies dadurch verdeutlicht, dass die Ellipse >Inlinehockey< etwas unterhalb der Ellipsen >Handball < und >Triathlon< lokalisiert ist.

3 Entgegen weit verbreiteten Vorurteilen beschränken sich ehemals elitäre Sportarten wie Tennis und Golf heute keineswegs auf die höheren sozialen Klassen, sondern werden seit langem von Angehörigen der mittleren und unteren Mittelklassen frequentiert. Ein wenig erwartetes Ergebnis der Bildungsexpansion besteht darin, dass diese Klassen nicht nur einen Aufstieg erlebten, was die Bildungstitel, sondern auch was die Sportbeteiligung angeht.

4 Die zunehmende Expansion des Sporttreibens wird - weit davon entfernt, zu einer flächendeckenden >Versportung ‘ des gesamten sozialen Raumes zu führen - vorwiegend durch die Integration von Frauen und bislang sportferner Altersgruppen getragen (vgl. Lamprecht/Stamm I994: 476). Gleichzeitig bleibt der Sport insgesamt eine »eher mittelschichtspezifische (und oberschichtspezifische) Angelegenheit« - obschon »mehrfach belegt«, wird dieser >Klassencharakter des Sportes »noch nicht von allen mit dem Sport Befassten geglaubt« (Winkler I995: 277).

5 Vgl. dazu besonders das von Vester et al. beschriebene »hedonistische Milieu « (200I: 52Iff.).

6 Die Triathleten gerieren sich als die - im Vergleich mit den Inlinehockey-Spielern - schärferen Gegner des Vereins, weil sie 
mit dem Vereinssport ständig konfrontiert sind und selbst noch mit einem Bein in der Vereinskultur stehen.

7 Bei einem solchen, die Sozialraumanalyse hermeneutisch nutzenden Verfahren geht es Bourdieu zufolge darum, ausgehend vom besonderen Ort, »den der Befragte im Sozialraum einnimmt, [...] ein generelles und genetisches Verständnis der Existenz des anderen anzustreben, das auf der praktischen und theoretischen Einsicht in die sozialen Bedingungen basiert, deren Produkt er ist: Eine Einsicht [...] in die untrennbar verwobenen psychischen und sozialen Prägungen, die mit der Position und dem biographischen Werdegang dieser Person im Sozialraum einhergehen « (Bourdieu I997: 786).

8 Die prozentualen Wachstumsraten der Bildungsberufe fallen umso geringer aus, je höher diese Berufe in der Berufsbereichshierarchie angesiedelt sind (Vester et al. 200I: 4II). »Bei den Bildungsberufen nehmen am stärksten Real-, Volks- und Sonderschullehrer $\mathrm{zu}$, gefolgt von den Gymnasiallehrern und den Hochschullehrern« (Vester et al. 200I: 4I2).

9 Bourdieus eigenes theoretisches Interesse galt eher dem Funktionieren stabiler, kontinuierlicher Habitus. Dies trug ihm den Vorwurf ein, seine Konzeption sei insgesamt statisch; deren dynamischen Aspekte gerieten aus dem Blick. Für eine entsprechende Diskussion der Habituskonzeption vgl. Alkemeyer/ Schmidt (2003).

Io Foucault hat diesen Gedanken in seinen späten Schriften über die Selbstsorge immer wieder, oft implizit geäußert, gelegentlich, in Interviews, aber auch deutlich ausgesprochen; vgl. insbesondere das Gespräch mit C. Baker aus dem Jahr I984 (Foucault I994).

II Diese Akzentuierung geht freilich über das von Bourdieu ausgearbeitete Konzept des Habitus hinaus.

I2 Wie Beate Krais in ihrer Erläuterung dieser Bourdieuschen These hervorhebt, existiert eine Klasse oder soziale Gruppe »nicht allein kraft >objektiver< Eigenschaften oder Merkmale, die bestimmten Personengruppen gemeinsam sind, und sie ist nicht einmal hinreichend definiert durch die Beziehungen zu anderen Klassen und Gruppen. Damit eine soziale Gruppe oder 
Klasse real existiert, bedarf es symbolischer Kämpfe, zunächst einer klassifikatorischen Praxis der Subjekte, in der es um die Durchsetzung der eigenen Sichtweise der sozialen Welt geht, vor allem aber einer Repräsentation der Klasse oder Gruppe im politischen Raum« (Krais I990: 34I).

I3 In seiner Studie über die französischen Cadres hat Boltanski (I990) nachdrücklich auf die »gesellschaftliche Arbeit« (Boltanski I990: 47) hingewiesen, die einer sozialen Gruppe erst ihre Gestalt gibt und sie sichtbar macht. Die Gestalt einer sozialen Gruppe kann analytisch erklärt werden, »indem man nach der Arbeit des Sich-Gruppierens, des Ein- und Ausschließens fragt, deren Ergebnis sie ist, und die gesellschaftliche Definitions- und Abgrenzungsarbeit analysiert, die mit der Bildung der Gruppe einhergegangen ist und die durch Objektivierung dazu beigetragen hat, sie als etwas existieren zu lassen, was sich von selbst versteht« (Boltanski I990: 47). Eine Gruppe von Akteuren mit ähnlichen sozialen Existenzbedingungen gewinnt Gestalt erst, indem sie »an sich selbst eine Neudefinitions- und Repräsentationsarbeit [vollzieht, d. Verf.]« (Boltanski ı990: 47).

Vgl. zur kulturellen Repräsentation und Eigendarstellung der kleinbürgerlich-mittelständischen Wohnbevölkerung des Berliner Wohngebietes Siemensstadt durch das Vereinsleben im SC Siemensstadt Gebauer/König (I986).

I5 »Sein [des Kleinbürgers, d. Verf.] Zuhause ist aber in Wirklichkeit das Reich der apolitischen Normalität. [...] In der gepflegten Privatheit von Familie, Haus und Garten, wie sie das biedermeierliche Idyll als statischen Gegenentwurf zum bedrohlichen Gewühl des modernen Massenzeitalters bietet, fristet der Kleinbürger ein sich dezidiert unpolitisch gebendes Leben - solange man ihn in Ruhe lässt. Nicht einer Partei gilt sein Engagement, sondern dem Verein. Nicht gegeneinander, miteinander heißt es hier, und es geht um den guten Zweck; etwa um die Natur (vom Schreberverein bis zur Freikörperkultur) und um die >Brauchtumspflege< (vom Männergesangs- bis zum Schützenverein). Natur und Brauchtum aber sind die Sphären der bedrohten Normalität, zu deren Rettung sich der Kleinbürger immer berufen fühlt« (Franke I990: 214f.). 



\section{Treue zum Stil}

Spiele artikulieren und registrieren gesellschaftliche Veränderungen schneller und sensibler als soziale Institutionen und normative Systeme; sie sind Katalysatoren von Selbst- und Weltverhältnissen. In ihnen entwerfen die handelnden Subjekte neue Formen, bevor diese eine feste soziale Gestalt gewinnen. Wandlungen des Welt- und Selbstverhältnisses sind Ausdruck tiefer liegender gesellschaftlicher Veränderungsprozesse. Sie artikulieren sich häufig in ästhetischen Phänomenen und Prozessen, zu denen im weitesten Sinne auch die Spiele zählen, die in einer Gesellschaft gespielt werden. Neue Sportpraktiken wie Skateboarding, Inlineskating, Triathlon, Risikosport, die außergewöhnliche sinnliche Erfahrungen verschaffen, führen die gewandelte Bedeutung der Gegenwart an unzähligen Plätzen des Gewöhnlichen und Alläglichen vor Augen.

Die neuen Spiele zeigen, wie bühnenhaft der Sport geworden ist, welche sinnlichen Potenziale er heute entfaltet, wie sehr er dazu drängt, ästhetisches Ereignis zu werden und den Spielern die Rolle von Helden anzubieten. Wie alle Schauspiele verändern sie die Gegenwart, die sie in ihren Aufführungen darstellen. Aber anders als das historische Bühnenspiel (Boschert 200I) sind sie zu Medien der Veränderung geworden.

Die postkonventionellen Spiele sind Katalysatoren sozialen Wandels. In ihnen zeigen sich nicht nur Veränderungen, die auch in anderen gesellschaftlichen Bereichen vor sich gehen, sondern durch ihr Zeigen greifen sie in den Wandel selbst ein. Zum einen tragen sie 
erheblich dazu bei, diesen zu erkennen und zu verbreiten; zum anderen verschaffen sie ihm prägnante Formen und soziale Anerkennung. Der Blick auf diese Dynamik wird nicht zuletzt durch die Beharrungstendenz im Feld des Vereinssports verstellt. Die Zahl derer, die diesen Institutionen treu bleiben und sie den Erwartungen der Gegenwart anpassen, darf nicht unterschätzt werden. Nach wie vor gibt es eine große Zahl von Sportinteressierten, die sich in den traditionellen Institutionen bestens aufgehoben fühlen und gerade den festen Halt von Vereinsstrukturen, ihre Regelmäßigkeit und Verlässlichkeit zu schätzen wissen und ihre Angebote, wie Sporthallen, Übungsleiter, Geräte, Spielbetrieb, Wettkämpfe in Anspruch nehmen. Für alle jene, die nach traditionellem Wettstreit und Meisterschaften streben, ist der organisierte Sport unumgänglich.

Im Unterschied zu Deutungen aktueller gesellschaftlicher Entwicklungen verbindet sich im Sport die Abnahme institutioneller Bindungen keineswegs mit sozialer Desintegration. Es erscheint wenig angebracht, diese Veränderungen als ein Zerfallen des $\mathrm{Zu}$ sammenhalts der Gesellschaft zu verstehen. Was man vielmehr erkennen kann, ist einerseits eine neue Art von Spielgemeinschaften, andererseits eine verstärkte Hinwendung der Spieler zu sich selber. Freilich schließen sich diese nicht in ihre Innerlichkeit ein, wie das traditionelle Konzept der Subjektivität suggeriert. Zwischen Subjektivierung und Gemeinschaftsbildung besteht kein gegenseitiges Ausschließungsverhältnis, in dem sich Subjektivität nur auf Kosten von Gemeinschaftlichkeit entfalten könnte und umgekehrt. Vielmehr ergänzen und stützen sich neue Subjektivierungsformen und neue Formen der Gemeinschaftsbildung gegenseitig.

Unsere Beobachtungen von neuen Spielen zeigen, vor dem Hintergrund einer traditionellen Sportart gesehen, eine Reihe neuer Züge. Sie sollen im Folgenden unter drei Gesichtspunkten als Anzeichen sozialer Veränderungen unserer Gesellschaft interpretiert werden: als neuartige Körpergesten, als eine Sorge um den Stil und als eine soziale Dynamik, die Aktivitätsfelder erzeugt, die es bisher nicht gab. 


\section{Gesten}

In den letzten Jahrzehnten ist im Feld des Sports eine Fülle neuer Gesten entstanden. Diese werden in den neuen Spielen zu bestimmten Mustern zusammengefügt und öffentlich aufgeführt. Während im Vereinssport Gesten und Verhaltensmuster durch Vorgaben festgelegt sind, geht diese Formgebung in den neuen Spielen unter starker innerer Beteiligung der Mitspieler vor sich. Zwar treiben auch diese mit anderen gemeinsam Sport, aber jeder von ihnen macht sich seine persönlichen Vorstellungen von seinem Bewegungsstil, handelt nach seinem eigenen zeitlichen Rhythmus und will sich als selbstbestimmt erfahren. Während der Körpereinsatz im traditionellen Sport primär instrumentell und auf ein Ergebnis gerichtet ist, sind die Bewegungen hier verlaufsorientiert, experimentierend und fließend.

Die Beteiligten an den neuen Spielen wünschen sich ihre Körper nicht als effiziente Maschinen. Durch Bewegungen wie Gleiten und Drehen, durch hohe Geschwindigkeiten werden Gefühle des Losgelöstseins, des beherrschten Rausches hervorgerufen. Für den ästhetischen Bewegungs- und Selbstgenuss der Inlineskater ist es wichtig, dass die Bewegungen durch reibungsarm gelagerte Rollen leichter und schneller werden. Aber diese Gesten der Leichtigkeit sind nicht vollkommen neu, sondern mit alten, bekannten Erinnerungen verknüpft, vor allem mit solchen, die aus dem Traum kommen, aus den geträumten Erlebnissen des Fallens oder Schwebens (vgl. Alkemeyer/ Gebauer/Wiedenhöft 200I).

Tatsächlich sind die neuen Spiele durch große Freiheitsgrade und eigene Gestaltungsmöglichkeiten gekennzeichnet. Sie favorisieren Virtuosität, Maskierung, Rausch und den Auftritt vor Publikum. Neuartige Spielgeräte geben Anlass, die Bewegungsmöglichkeiten des menschlichen Körpers kreativ auszuloten. Zum einen wirken diese als Steigerung der Person, zum anderen haben sie, gemeinsam mit den anderen Zeichencodes, eine unübersehbare Bedeutung für die Identität und Zusammengehörigkeit der Spielgemeinschaften. Mit den neuen Spiel- und Bewegungsweisen verbinden die Akteure Vorstellungen eines wenig normierten Lebens, einer durch Einsamkeit und Freiheit geprägten Existenz des >Großstadtsingles<, der diese Unverbindlich- 
keit der neuen Spiele genießt. Vergnügen entsteht aus den ungeplanten Begegnungen mit Gleichgesinnten.

In den neuen Spielen treten, nach der Klassifikation von Caillois (I982), die Maske (mimikry) und die Suche nach dem Rausch (illinx) in den Vordergrund und verbinden sich mit dem Prinzip des Wettstreits (agon). Während im klassischen Wettkampf immer auch der Zufall (alea) eine Rolle spielt - Zufall des Ortes und der Zeit, der äußeren Bedingungen und der persönlichen Form -, hat die Kategorie der alea in den neuen Spielen ihre konstitutive Rolle verloren. In den Risikosportarten, die sich gegenwärtig zunehmender Beliebtheit erfreuen, wird der Zufall - aus gutem Grund - völlig auszuschalten versucht. Risikosport ist nicht Zufallssuche, sondern eine Wette der Teilnehmer mit sich selbst: dass sie die Herausforderungen des Spiels bestehen. Im Risikosport will der Athlet beweisen, dass er stärker ist als die Gefahr und auf diese Weise den Wert seiner Person demonstrieren. ${ }^{\mathrm{I}}$

Maske und Rausch als Leitkategorien der neuen Spiele stellen ein Problem für die theoretische Beschreibung der sozialen Organisation dar: Wie ist ein Zusammenhalt in einer Gruppe möglich, wie kann überhaupt eine Gemeinschaft entstehen, wenn diese darauf gerichtet ist, ein Maskenspiel zu organisieren und einen Zustand des Rausches zu erzeugen? Wie kann aus diesem subjektiven Einsatz etwas Objektives, ein die flüchtigen Situationen überdauerndes soziales Ganzes entstehen?

STIL

An den neuen Spielen lässt sich erkennen, wie sich die klassischen Methoden der Erzeugung von Disziplinen (Foucault) und der Ausbildung eines Habitus (Bourdieu) in der gesellschaftlichen Praxis verschoben haben. Beide Konzepte setzen voraus, dass die Körper der Akteure in praktische gesellschaftliche Prozesse eingespannt sind und in diesen eine erkennbare Form erlangen. Nun gibt es aber zunehmend mehr Handlungsbereiche, in denen die Körper auf den ersten Blick keine besondere Form ausbilden müssen (Gebauer 200I). In der Arbeit am Computer scheint der Körper weitgehend ungeformt zu 
bleiben; er wird oft demonstrativ als unbeschäftigt dargestellt. In den neuen Spielen hingegen werden die Körper demonstrativ beschäftigt. Hier bilden sie eigene Disziplinen aus, hier legen sich die Akteure einen neuartigen körperlichen Habitus zu.

Während in der traditionellen Arbeitsgesellschaft in der beruflichen Tätigkeit ein je spezifischer körperlicher Habitus ausgebildet wurde und die Freizeit Möglichkeiten bot, sich gehen zu lassen, faul und haltungslos zu sein, verhält es sich heute der Tendenz nach fast umgekehrt. Die Freizeit ist heute zu der Sphäre geworden, in der die Subjekte ihre Körper formen und ihrem Leben Gestalt und Haltung geben. Sowohl im Arbeitsleben als auch in der Freizeit haben sich die Weise und die Bedeutung der Körperformung verändert.

Die üblicherweise als sekundär eingestuften Bereiche der Nichtarbeit, der Freizeit und Muße rücken ins Zentrum der Gesellschaft. Gerade die Spiel- und Freizeitwelten stellen den Individuen ein schnell wachsendes Angebot an Modellen der Beschäftigung und Formung des Körpers zur Verfügung. In den von uns untersuchten neuen Spielen bilden die Teilnehmer körperliche Verhaltensmodelle und Habitusformen aus. Hier wird der Stil erworben, den die Subjekte in andere soziale Felder transportieren. Im Spiel ist der Ursprung eines Stils, dem die Teilnehmer wie einer persönlichen Signatur treu bleiben.

Die Suche nach einem passenden Stil kennzeichnet die in den neuen Spielen beobachtbare Hinwendung der Teilnehmer zu sich selbst. Der Stil greift über das Individuum hinaus; das Subjekt, das ihn verwirklicht, erzeugt etwas Überindividuelles. ${ }^{2}$ Im Stil entwirft sich ein Ich und macht den Entwurfscharakter der Person durch sein Spiel deutlicher als anderswo. Im Vereinssport hingegen steht das Spiel höher als der individuelle Stil. Das Spiel gibt die Form vor, in der sich die Einzelnen zu verhalten haben: gegen die Anderen, auf den Erfolg und ein messbares Resultat ausgerichtet. Bei aller Selbstbezogenheit ist der Wettkämpfer aber kein unbedingtes Ich: Er unterstellt sich dem Spiel, ordnet sich den Regeln unter und kooperiert mit den anderen (vgl. Gebauer 2002).

Im Unterschied zur Achtung des Spiels und seiner Regularien entsteht die Treue zum Stil aus einer inneren Haltung, nicht aufgrund von außen kommender Verpflichtungen. In der Gemeinschaft, die 
durch denselben Stil zusammengehalten wird, besteht stillschweigendes Einvernehmen über das, was sich gehört und was nicht. Aber explizit formulierte Geschmacksregeln existieren nicht. Der Eintritt in eine Spielgemeinschaft geschieht durch individuelle Akte einer Geschmacksprüfung: Passt der neu in die Gemeinschaft Eintretende zu dieser? Ist er das, was man in der Gruppe unbedingt zu sein hat: >cool«? Die Zugehörigkeit zu demselben Stil wird auf der Grundlage eines ungeschriebenen Kodex und einer sehr empfindlich wahrnehmenden >sozialen Ästhetik entschieden. Vor allem darf der Neue keine Angestrengtheit erkennen lassen; sein Können muss natürlich, ungelernt und mühelos erscheinen.

Der soziologische Beobachter ist im nachhinein in der Lage, bestimmte Regularitäten der Geschmackswahlen zu rekonstruieren; die Spieler hingegen handeln nicht nach aufgestellten Regeln, sie leben sich in ihren Stilen aus. Was sich im Spiel gehört und was nicht geht, bestimmen sie immer wieder aufs Neue, und in ihren Neubestimmungen ist derselbe Stil wiedererkennbar. Er ist in der gesellschaftlichen Spielpraxis verankert und an die Position der einzelnen Spieler im sozialen Raum gebunden. In den neuen Spielen wird das Prinzip der sozialen Geschmackswahl auf die Spitze getrieben. In Prozessen der Kooptation wird eben die Person vorgezogen, die den Wählenden als die sam besten Passende< erscheint. Bei diesem Vorgang handelt es sich um ein im Habitus angelegtes distinktives Vermögen, einen »praktischen Sinn« (Bourdieu), der keiner expliziten Abwägungen und Entscheidungen bedarf.

In den vorangegangenen Kapiteln ist der Stil, den wir in den neuen Spielen beobachten, ausführlich beschrieben worden. Er ist eine Weiterarbeit am ausgebildeten Habitus jener Klassenfraktion, der die handelnden Subjekte angehören. Stil bezeichnet eine formende Tätigkeit, die drei Ziele anstrebt: soziale Neuheit der Personendarstellung, Subjektivierung der Werte und Normen des Verhaltens, Heroisierung des Ichs mit Hilfe besonderer Verhaltensweisen, denen man auch im Alltagsleben treu bleibt. Das zweite und dritte Ziel unterscheiden sich von den soziologischen Kennzeichen der »Stile des Lebens«, die Soeffner (200I) angegeben hat.

In der soziologischen und anthropologischen Literatur, auf die sich Soeffner bezieht, insbesondere auf Simmel (I995) und Plessner 
(I98I), wird der Stil, den ein soziales Subjekt seinem Handeln, ja seinem Leben gibt, als eine »Entlastung und Verhüllung des Persönlichen« (Simmel I993: 382, vgl. Soeffner 200I: 89) angesehen. Sie ist »das Heraustreten aus der Einmaligkeit« und bedeutet »den Anschluß an etwas Allgemeines « (Soeffner 200ı: 83). Insofern ist die Überzeugung der Mitglieder von Stilgemeinschaften, sie würden hier ihre >eigentliche< oder >authentische< Individualität ausprägen, ein grundlegender Irrtum: sie »erliegen der Paradoxie des kollektiven Individualismus« (Soeffner 200I: I04). Diesen Irrtum weist Soeffner an zwei Beispielen nach, die er unter dem Gesichtspunkt ihrer politischen Wirkungen untersucht, dem Wandervogel und den Ravern.

Die von uns beobachteten neuen Spiele weisen einen Stilbegriff auf, der in vielem der Techno-Kultur, wie sie Soeffner beschreibt, ähnlich ist; aber aufgrund der beiden Tendenzen der Subjektivierung und Heroisierung erhält der Stil bei ihnen eine neue Bedeutung. Die Tendenz der Subjektivierung lässt sich bei ihnen nicht im Sinne einer in die Irre laufenden Individualisierung interpretieren. Bei den Teilnehmern an den neuen Spielen steht die Bemühung im Vordergrund, eine spezifische Bewegungsweise körperlich und stilistisch zu erwerben und weiterzuentwickeln, die sie über den Status des Individuums erhebt.

Bei den in der Literatur beobachteten und diskutierten Fällen neuer Stilbildungen ging es darum, die Anforderungen des klassischen Personenkonzepts in ausgezeichneter Weise zu erfüllen: um die Suche nach einer besonderen Markierung von Individualität, die mit Hilfe eines Stils gerade verfehlt werden musste. Bei den neuen Tendenzen scheint es gar nicht mehr um das klassische Personenkonzept der Authentizitätssuche zu gehen. Das Exzessive, Maßlose, das Verrückte, die Verschmelzung von Mensch und Technik, die selbstverständliche Vereinnahmung von öffentlichen Räumen, die performativen Aktivitäten, die die anderen Bewohner der Öffentlichkeit in die Rolle von Zuschauern drängen, zeigen eine Entschlossenheit, die Sphäre der gewöhnlichen Menschen zu übersteigen. Unsere Zeit, die die Verbesserung des Menschlichen in vielen technischen Formen diskutiert, kann in diesem Ziel keine Hybris mehr erkennen. 


\section{COOLNESS}

Die Spielgemeinschaften werden nach dem Prinzip eines relativ homogenen, von den Eingeweihten leicht wiedererkennbaren Stils gebildet, der eine das Verhalten der Teilnehmer insgesamt kennzeichnende Eigenschaft generiert, die mit dem Ausdruck >Coolness < bezeichnet wird. Man kennt eine solche vereinheitlichende Verhaltenscharakteristik von Zirkeln der Oberschicht, von Dichtergruppen, altehrwürdigen akademischen Colleges, von den Pariser Grandes Ecoles etc. ${ }^{3}$ Sie gilt als Merkmal eines bestimmten Elitismus; sie kennzeichnet eine bestimmte >Aristokratie< des ästhetischen Verhaltens, die freilich nicht unbedingt an einen hohen Stand gebunden ist. Als >aristokratisch < gilt das besondere Stilvermögen der Gruppenmitglieder, insofern es sich in einen Gegensatz zum >Gewöhnlichen< stellt.

Wenn Jürgen Trabant Recht hat, tritt >Coolness< heute die Nachfolge jenes Werts an, der in der höfischen Literatur der Frühen Neuzeit unter dem Begriff der Sprezzatura gefasst wurde, eine Bezeichnung, die Baldessar Castiglione in seinem Cortegiano eingeführt hat. »Sprezzare heißt eigentlich >verachten, nicht beachten $<$. Sprezzatura heißt also >Verachtung<, >Nicht-Beachten<. Gemeint ist damit: NichtAchten auf die eigene Handlung. Das ist aber natürlich nur dann möglich, wenn die Handlungsweise völlig sicher beherrscht wird. [...] Die Franzosen haben sprezzatura mit Nonchalance übersetzt, in der deutschen Übersetzung des Cortegiano finde ich Lässigkeit. Das ist nicht schlecht, aber ich denke, die beste Übersetzung ins heutige Deutsch [...] wäre coolness« (Trabant 200I: I68).

Sprezzatura ist eine Kunst, die sich verbirgt; sie ist allen sichtbar, sie wird gezeigt, geschätzt, bildet die Grundlage der Beurteilung von sozialem Verhalten, aber sie wird nicht als Kunst vorgeführt, sondern als ein natürlicher Besitz. Die Kunst des Verhaltens ist ein altes aristokratisches Ideal. Wer es heute zum Maßstab seines Handelns nimmt, versteht sich als Mitglied einer sozialen Elite. Die Teilnehmer an den neuen Spielen betrachten sich als eine Auslese und als Auslesende. Mit dem Willen zum Stil wird eine soziale Sonderrolle ausgedrückt: ein Bessersein. Sie geht noch über die Distinktion hinaus, insofern sie nicht nur Grenzen gegenüber anderen Gruppen zieht, sondern ein höheres Wissen und Können auszeichnet und als >natürlich< verbürgt. 
Diese erworbene >Natürlichkeit< wird den anderen außerhalb der Gruppe stehenden Personen zur Bewunderung angeboten. Sprezzatu$r a$ als Stil wird über die verschiedenen Felder des sozialen Handelns verbreitet. Der in den neuen Spielen herausgebildete Stil der Coolness tritt hier in vielen Variationen auf, und zwar unter den Bedingungen des jeweiligen Feldes. Die Herkunft des Stils aus den Spielen erkennt man daran, dass in all seinen Variationen das Merkmal der Sportlichkeit auftritt und sich mit anderen Bereichen und Attributen (Mode, Erotik, Kunst, Popkultur etc.) mischt.

\section{Muster}

Die ungewöhnliche Mischung von Praktiken ist eine Wirkung des Stils. Der Triathlon ist das beste Beispiel dafür; er stellt eine Kombination von Sportarten dar, die sich in der Perspektive eines traditionellen Sportverständnisses gegenseitig ausschließen - man ist entweder Radrennfahrer oder Schwimmer oder Läufer und nicht alles gleichzeitig. Die für den Triathlon typische Vereinigung konträrer motorischer Anforderungen wurde, wie der Gründungsmythos dieser Sportart besagt, >am Reißbrett< konstruiert, als eine Wette, etwas zu tun, >was eigentlich nicht geht<. Mittlerweile hat sich Triathlon in einen Stil verwandelt. Die Sportpraxis ist ver-rückt, aber insofern diese einen Stil bildet, gelten seine Teilnehmer als >normale< Menschen, die einen anerkannten und begehrten Stil verwirklichen.

Die unlösbar erscheinende Herausforderung dieser Praxis ist die Bedingung dafür, dass sie Helden hervorbringt. Triathlon hat aus seiner internen Widersprüchlichkeit eine Mythologie gemacht. Ähnlich ist es mit den Ultralangläufern und Risikosportlern. Das Stilprinzip, Unmögliches miteinander $\mathrm{zu}$ verbinden, findet man auch in anderen Bereichen: ein erfolgreicher Unternehmer umrundet als Ballonfahrer die Erde, ein ehemaliger Fußballspieler stellt sich als Finanzexperte dar, ein anderer als Geschäftmann im Dienst der Nation, ein alternder Bodybuilder wird zum Filmstar, dann zum Gouverneur. In der Nachfolge des Dandys früherer Zeiten, der seine Person mit exzentrischen Praktiken zu einem Stilvorbild machte, sichern sich die Teilnehmer an den neuen Spielen die Anerkennung der Gesellschaft. 
Warum feiert man das Zusammenfügen von Unvereinbarem? Wenn eine solche Praxis sich als ein Stil durchsetzt, wird eine neue Vorstellung von der Person erzeugt, ein neues Markenzeichen des Menschen. Dieser tritt jetzt in neuen hautengen Materialien auf, als eine bunte, grelle, superschlanke Figur mit asketischen Gesichtszügen und langen, definierten Muskeln. Er bewegt sich wie im Traum auf seinem Supergerät. Der Athlet, der einen neuen Stil verwirklicht, wird zu einem Bild. Er erscheint als technisiertes Wesen, teils aus neuen synthetischen Materialien, teils aus Blut und aus Fleisch, das in neuen Verfahren gehärtet wurde. An einen solchen Körper kann man Maschinen anpassen, die zum gleichen Bild gehören. Triathlon ist das Bild grenzenloser Passfähigkeit des Menschen und der Verlebendigung der Maschine. In seinem Kontext wird dieser Symbiose von Mensch und Maschine nicht mit Skepsis begegnet; sie gilt als ein Triumph des Körpers, der sich in Hochtechnologie integriert und auf diese Weise zu extremen Leistungen fähig wird.

Als ein Bild davon, was Menschen aus sich machen und von sich verlangen können, dient der Triathlet vielen Bereichen als Vorbild für eine bestimmte Leistungsfähigkeit. Die Sportart zeigt exemplarisch, wie ein Ich dadurch, dass es ein vorgegebenes Muster erfüllt, $\mathrm{zu}$ einem Helden werden kann. Im Zentrum der neuen Sportarten finden wir neue Muster des Handelns. Handlungsmerkmale wie diese gibt es auch außerhalb des Sports; dort sind sie unscharf, kaum erkennbar. Die unauffälligen Variationen dieser Merkmale in den Alltagspraktiken werden sichtbar, wenn man das Muster des Spiels wie eine Folie darüber hält und sie gleichsam durchscheinen lässt. Es zeigen sich dann untergründige Verwandtschaften. Für einen Stil gibt es Muster und Variationen; üblicherweise geht das Erkennen der Variationen vom Muster aus. Aber man kann auch, in einem gegenläufigen Vorgang, von den Variationen ausgehend das Muster identifizieren. Ebenso wie das Muster die Variationen klärt, klären diese das Muster. Daher kann man ein Muster auch an den Handlungen des Alltagslebens erkennen. Aber die Muster der neuen Spiele haben aufgrund ihrer Körperlichkeit und Sichtbarkeit eine besondere Eignung für die Mustergebung. ${ }^{4}$

Die Spielgemeinschaften werden von der Treue zum Stil, den sie entwickelt haben, und von dem Glauben an die Auszeichnung, welche 
die Aneignung des Stils verleiht, zusammengehalten. Der Stil vereinigt die Mitglieder dieser Gruppen auch in Bereichen außerhalb des Spiels. Inlineskater ebenso wie Triathleten erkennen sich auch in der Alltagspraxis als ihresgleichen. Was auf den ersten Blick aussah wie das Auseinanderfallen der Gesellschaft, erweist sich so als eine neue Weise der sozialen Organisation und Zusammengehörigkeit.

\section{Figuren}

Ein Stil, der seine Konstruktionsprinzipien verbirgt, fordert Anerkennung von der Gemeinschaft. Angesichts eines exemplarischen Könnens reagiert diese mit Bewunderung und Verehrung; die Gruppe wird zur Gemeinde (Gebauer I999). Sie ordnet sich den herausragenden Figuren unter, die eine exemplarische Darstellung des kollektiven Stils vorführen. Als >Figuren < können wir sie bezeichnen, weil sie in dem Maße, wie sie zu einer Verkörperung des Stils werden, das Individuelle und Persönliche übersteigen und sich zu einem Ausdruck des Allgemeinen machen, welches die ganze Gruppe kennzeichnet. Ein Stil gibt der Figur etwas Überpersönliches; er wirkt wie eine Maske.

In dieser Sicht führen die neuen Spiele, die sich gerade durch ihre Subjektivierung auszeichnen, zu einem Zurücktreten des Persönlichen und Individuellen zugunsten eines gemeinschaftlichen Musters. Eine solche Gemeinschaft kann ausschließlich mit Bezug auf das Muster aktualisiert werden; persönliche Vertrautheit ist dafür nicht notwendig. Um Gemeinschaftsprozesse in Gang zu setzen, reicht aus, dass die Teilnehmer das Muster kennen und fähig zur Darstellung und Erkennung des Stils sind. Wenn sich zwei wildfremde Moutainbiker im Wald begegnen, grüßen sie sich, als kennten sie einander; sie haben mit einem Blick erfasst, dass der andere >dazugehört<: Sein Fahrradrahmen, die Bremsen, die Kleidung, die Fahrweise sind Züge eines Stils, dem auch der andere treu ist. In ihren Erzählungen tauchen die anderen nicht mit ihren Persönlichkeitsmerkmalen auf, sondern werden anhand des gemeinsamen Musters erkannt - der andere ist der mit dem Carbonrahmen. Das Muster, das die Teilnehmer der neuen Spiele zusammenhält, lässt zwischen ihnen keine Wärme entstehen; sie halten in der Nähe ihrer Selbstentwürfe Abstand. 
Mit ihrem wie eine Maske wirkenden Stil zielen die neuen Spiele über die gegebene Gesellschaft hinaus. Der von den Teilnehmern gelebte Stil gestaltet ihre Beziehungen zur Gesellschaft um; er führt dazu, dass Bindungen und Verpflichtungen reflektiert, traditionelle Einstellungen überdacht werden. So wird im Triathlon die Haltung der Askese eingenommen, aber nicht im Sinne einer religiös geprägten Distanz zur Welt oder als äußerste Zurückhaltung in weltlichen Dingen, sondern als Verausgabung von Kraft und Energie und als Verschwendung von Zeit, Raum, Geld und Technik. Ziel ist nicht eine Verinnerlichung der Person, eine Konzentration, Umlenkung auf spirituelle Ziele, sondern ein optimaler Konsum von mühsam angesammelten physischen und materiellen Ressourcen, um den Körper zu erhöhen. Auf diese Weise strebt das Subjekt eine Steigerung seines gesellschaftlichen Werts an.

\section{HELDEN}

In dieser Perspektive gehen die in den neuen Spielen gemachten Gewinne an Handlungsfähigkeit und sozialer Dynamik auf Kosten der traditionellen Vereinssportler. Den interviewten Handballerinnen ist bewusst, dass sie mit ihrer vehementen Ablehnung der Suche nach einem Stil eine konventionelle Position einnehmen. Ihr Protest richtet sich gegen den sunernsten< Gestus und gegen das Maskenhafte des Stils. Beides erscheint ihnen nicht >echt $<$ sie suchen >das Wahre $<$ und finden es in einer Sportpraxis, die sie auf den Erfolg der Mannschaft und auf das höhere Interesse des Vereins verpflichtet.

Gegen diese Form der Beschneidung des Handlungsspielraums rebellieren insbesondere die Inlinekater: Sie organisieren ihr Spiel selbst; sie genießen ihre Fähigkeit, dies durch improvisierendes Handeln zu schaffen. Bei ihnen gibt es nicht mehr die Unterscheidung zwischen >Echtheit< und >Gespieltem $<$; sie favorisieren ein spielerisches Weltverhältnis und schätzen dies ungleich höher ein als auferlegte Strukturen und Reglements. Bei ihnen findet man ein Pathos des Improvisierens und der Bindungslosigkeit, das in den schwebenden Bewegungen dieser Sportpraxis körperlich evident wird (vgl. Alkemeyer 2003a). Es geht ihnen um die Kontrolle über das eigene 
Leben, eine Kontrolle im Sinne der Ablehnung von allem, was dem Spieler zu nahe kommen und seinen Stil gefährden könnte.

Man kann diese Haltung als eine Suche, nicht nach dem guten Leben, sondern nach guten Gelegenheiten ansehen, die ihnen die neuen Spiele, die technischen Geräte, der großstädtische Raum, die Mitspieler und Zuschauer, diese ganze soziale Szenerie zu bieten vermag. Die Spieler zeigen einen demonstrativen Konsum der gebotenen Chancen und die Fähigkeit, diese für sich zu nutzen. Ihr Vergnügen geht über eine hedonistische Haltung hinaus; nicht zuletzt entspringt sie auch einem umfassenden Können, der sprezzatura; sie gibt dem Spieler die Möglichkeit, ein anderer zu werden. Er richtet sich gegen seine alte Normalität, ohne die gesellschaftlichen Verhältnisse infrage zu stellen.

Auch die Triathleten verfolgen ein Projekt der radikalen Selbstbezüglichkeit; sie sind aber nicht bereit, die Kategorie des >Echten < aufzugeben. Im Gegenteil suchen sie nach neuen Formen der Echtheit, die weit über jene der Vereinssportlerinnen hinausgehen. Bei ihnen dreht sich alles um das eigene Ich; die Wahrheit der eigenen Person soll getestet, geprüft werden: Hält sie die gesuchten Belastungen aus oder nicht? Daher wird die Askese wichtig; sie erhält eine ethische Dimension. Die Athleten entwerfen sich als Vorläufer einer neuen Stufe des Menschlichen, eines Größerwerdens des Ichs: Der Sportler löst sich von seinem früheren Leben und strebt danach, eine neue, bessere Person auszubilden und über seine soziale Existenz hinauszugehen. Auf der Suche nach einer Verbesserung seines Ichs erhöht er sich zu einem Helden. Mit dem Aufsetzen einer Maske ent-subjektiviert er sich in ein Heroentum, das auf die Stützung durch die Gemeinschaft angewiesen ist.

Das Entstehen solcher gewöhnlichen Helden lässt sich in vielen gesellschaftlichen Bereichen beobachten. Gewöhnlich sind sie, weil sie nicht die Macht der großen Helden besitzen, eine neue Möglichkeit in die Welt zu bringen, den Horizont zu öffnen oder ihre Gegenwart herauszufordern. Vielmehr entstehen sie als Figuren eines Stils. Sie sind nicht Helden aus eigener Kraft, sondern Effekte in Spielen, die auf die Hervorbringung von Bildern und Masken hin angelegt sind. 


\section{Stil-Aristokratie}

Spiele sind ein wichtiger Ort in der Gesellschaft, an dem Neues hervortritt. Dies geschieht in unvorhersehbaren Prozessen, die aus der Mitte von Spielgemeinschaften auftauchen. Der Stil, der in den neuen Sportarten ausgebildet, eingeübt und zur zweiten Natur gemacht wird, ergreift den ganzen Körper und gestaltet dessen Bewegungsweisen dauerhaft um. Er ist eine Körperformung, die den Bewegungen wiedererkennbare und sozial bewertete Merkmale gibt. Obwohl die Bewertungsschemata des körperlichen Handelns historisch entstanden und wandelbar sind, haben sie eine erstaunliche geschichtliche Kontinuität und Verbreitung über die Kulturen. Der Gegensatz von shohem < und >niedrigem < Stil findet sich in je eigener Ausprägung in vielen historischen Epochen und regionalen Kulturen vor. Hoher Stil wird mit der Kennzeichnung des >Noblen<, niedriger Stil mit jener des >Gewöhnlichen< assoziiert. Diese Unterscheidung stellt das Gekonnte, Beherrschte, Elegante, das durch Leichtigkeit und Lässigkeit gekennzeichnet ist, in einen Gegensatz zum Schwerfälligen und Bemühten. Ein so aufgefasster Stil einer bewegungsästhetischen >Aristokratie wird aufgrund virtuoser Könnerschaft erworben und bewundert.

Goffmans Begriff der Rollendistanz (Goffman 1973) ist eine genaue Kennzeichnung dieses Stils: Im Unterschied zu einem Verhalten, das ängstlich auf Korrektheit und Sicherheit bedacht ist, führt es atemberaubende Kunststücke vor, das Loslassen von Halt, wo andere sich an die Griffe klammern, lässige Bewegungseinschüsse, fast tänzerisch, ohne jemals das Gleichgewicht zu verlieren. Freilich übersieht Goffman, dass ein solcher feiner Stil nur den Virtuosen der sprezzatura und der Coolness möglich ist. Tatsächlich entsteht bei den Zuschauern der Eindruck von Distanzierung, aber diese ist keine Entfernung von einer sozialen Rolle, sondern von der braven Erfüllung gegebener Anforderungen. Der Könner verwandelt das, was andere mit Bedacht und nach Vorschrift ausführen, in einen leichtfüßigen Tanz.

Jeder Stil verkörpert sich in idealer Weise in einer vorbildlichen Person. Aufgrund ihres perfekten Stils steht diese im Zentrum ihrer Gemeinschaft, wenn sie in Erscheinung tritt: In einer Skatehalle vollführen junge Inlineskater und Skateboarder in einer Half-Pipe etwas 
angestrengt ihre Kunststücke, bis eine Person auftaucht, die sich bis dahin im Hintergrund gehalten hat. Ein junger Mann, ohne Knie- und Handgelenkschoner, mit nacktem Oberkörper stellt sich an den Rand der Bahn. Scheinbar in sich versunken, unberührt von der plötzlichen Aufmerksamkeit, streift er sich in lässiger Haltung mit den Händen über die Unterarme: Der emsige Betrieb kommt sofort zum Erliegen; alle schauen auf die einsame Gestalt, die sich plötzlich ohne jedes Vorzeichen in die Half-Pipe stürzt. Was er seinem Publikum zeigt, übertrifft alles bis dahin Vorgeführte an Gekonntheit, Kraft, Eleganz und Wagnis. Ebenso unvermittelt, wie seine Darbietung begonnen hat, endet sie: er steht wieder am Rand der Rampe, unbeteiligt, unbewegt, gelangweilt, nicht im Mindesten erschöpft. Niemand weiß, was er denkt, und wann er eine neue Probe seines hohen Könnens geben wird, worauf alle Beteiligten geradezu sehnsüchtig warten.

Es gibt viele solcher Helden in den neuen Sportarten. Ihre zentrale Bedeutung für die Stilgemeinschaften ähnelt dem aristokratischen Prinzip der höfischen Gesellschaft. Solche Gemeinschaften ordnen sich um einen Mittelpunkt. In ihnen herrscht die Überzeugung, dass ihre Mitglieder eine Elite darstellen. Nach ihren eigenen Kriterien stehen sie höher als all jene, die ihr Spiel nicht beherrschen. Sie besitzen ein Zentrum, das vom Stilvorbild gebildet wird, um das herum sich die anderen Mitglieder in konzentrischen Kreisen einordnen, bis hin zu den Positionen an der Peripherie. Der Vereinssport ist durch andere Machtverteilungen gekennzeichnet. Seine offizielle Rangfolge ${ }^{5}$ wird anders hergestellt: Die Athleten müssen ihre Rangplätze in Wettkämpfen erwerben und verteidigen. Das Leistungsprinzip bewertet die Resultate von Handeln und schreibt dieses der Person als Verdienst $\mathrm{zu}$.

Bei der Bewertung mit Hilfe von Stilprinzipien kommt es hingegen nicht in erster Linie auf das Ergebnis der Handlung, sondern auf die Form an. Alle Spieler bewundern das Stilvorbild und erwarten, dass sie selbst von den Außenstehenden bewundert werden, weil sie fähig sind, ihren Stil zu verwirklichen. Ihre Zugehörigkeit zu einer Stil->Aristokratie< führt zu einer Erhöhung ihres Selbstverhältnisses, die Sichtbarkeit der Spiele demonstriert die Macht des Stils.

So kommt es, dass der Triathlet, unabhängig von seiner Leistungshöhe, schon weil er Triathlet ist, Anerkennung verbuchen kann: 
weil er die drei Sportarten, die methodische Lebensführung, die sportliche Fachkenntnis, die Technologie, den Kleidungscode, kurz: weil er einen exzentrischen Stil beherrscht. Obwohl es in diesem Stil sowohl Elemente der Askese als auch des sinnlichen Genusses gibt, lässt er sich weder als protestantische noch als hedonistische Gesinnung ausweisen. Der saristokratische< Stil entsteht vielmehr aus dem Wunsch nach intensiver sinnlicher Erfahrung und stellt sich unter einen zivilisatorischen Selbstzwang, der eine scharfe Selbst- und Fremdbeobachtung ausbildet.

Im Leistungssport müssen die Inhaber von Führungspositionen ihre Überlegenheit in einem permanenten Kräftemessen immer wieder aufs Neue beweisen. Auch das Stilvorbild in den neuen Spielen muss sein Besser-Sein darstellen, es ist aber durch seine Bewunderer, die etwas vom Glanz seines perfekten Könnens auf sich selbst zu lenken hoffen, vor permanenten Leistungsmessungen und -vergleichen, wie sie im Verein üblich sind, geschützt.

Die Beherrschung eines Stils ist ein Vermögen, das nicht unmittelbar in andere Kapitalsorten konvertierbar ist. Wir haben aber gesehen, dass sie Veränderungen der sozialräumlichen Position in der horizontalen Dimension bewirken kann. Die Teilnahme an neuen Spielgemeinschaften kann in diesem Terrain neue Aktionsfelder erschließen. Spiele sind ihrer Außenwelt gegenüber empfindlich, insofern sie wie poröse Gebilde die sich dort vollziehenden Veränderungsprozesse aufnehmen. Weil Spiele symbolische Welten darstellen, die sensitiv für ihre Umgebungen und dabei in sich relativ geschlossen sind und eigene Sinnstrukturen besitzen, begünstigen sie die Ausbildung besonderer Selbst- und Weltverhältnisse. In den von uns untersuchten Spielen äußern diese sich als eine neue Art von Elitismus.

Angesichts der zum Stillstand gekommenen vertikalen Mobilität in der Gesellschaft hat dieser Elitismus den soziologischen Sinn, die Binnendifferenzierung, man könnte auch sagen: die Spaltungen auf der horizontalen Achse deutlicher hervortreten zu lassen. In der von hoher Dichte gekennzeichneten mittleren sozialräumlichen Region werden neue Spielformen mit einem hohen Anspruch auf die ästhetische Kategorie des Stils gepflegt und der Körper wie ein Kunstwerk zur Betrachtung angeboten. Im Gedrängel der kleinbürgerlichen Klassenfraktionen wird die quasi-artistische Bearbeitung von Leib und 
Leben zu einem handfesten Argument für den Anspruch, einer neuen Art von Elite anzugehören. Treue zum Stil hat vor dem Hintergrund der Schließung des sozialen Raumes einen doppelten Sinn: Mit der Abgrenzung nach außen bewirkt und festigt sie eine Gemeinsamkeit der Haltung im Inneren.

\section{ANMERKUngen}

I Das Risiko ist, wie sich im Anschluss an die Studien von Nerlich (I997) und Bonß (I995) festhalten lässt, ein spezifisches gesellschaftliches Konstrukt zur Bewältigung des universalen Problems der Ungewissheit, das historisch erstmals im Kontext des Fern- und speziell des Seehandels im I4. Jahrhundert gebildet wird. In der sozialen Konstruktion von Unsicherheit als Risiko wird diese nicht (mehr) als schicksalhafte Bedrohung angesehen, sondern als ein zu- und be-rechenbares Wagnis, das sich nur dann negativ bemerkbar macht, wenn falsch kalkuliert und gehandelt wird. D.h. im Risikodiskurs wird von einem Subjekt ausgegangen, das sich der Unsicherheit stellt, anstatt ihr ausgeliefert zu sein, ja diese auch absichtlich und durchaus lustvoll herausfordert, um sie zu meistern.

2 Vgl. zum Verhältnis von Stil, Selbst und Gemeinschaft Soeffner (I986, I995, 200I) und Hahn (1986).

3 Dort treten die Verhaltenscharakteristiken freilich unter anderen Bezeichnungen auf; man spricht dort von >Nonchalance<, >Gelassenheit<, >Distanziertheit<, >Natürlichkeit< u.ä.

4 Diese Verschränktheit von Muster und Variation ist charakteristisch für einen künstlerischen Stil; vgl. Bateson (I985a: 206208).

5 Daneben gibt es auch in informellen Auseinandersetzungen hergestellte Hackordnungen (vgl. III). 



\section{Literatur}

Adamowsky, Natascha (2000): Spielfiguren in virtuellen Welten. Frankfurt a.M.: Campus.

Alkemeyer, Thomas (2003): Semiotische Aspekte der Soziologie: Soziosemiotik, in: Roland Posner/Klaus Robering/Thomas A. Sebeok (Hg.): Semiotik (Semiotics). Ein Handbuch zu den zeichentheoretischen Grundlagen von Natur und Kultur. Berlin/New York: de Gruyter, S. 2757-2846.

Alkemeyer, Thomas (xxxx): »Verkörperungen. Über die Aufführungen gesellschaftlicher Selbst- und Weltbilder im Sport«, in: Bernd Wirthus (Hg.): Fiktion und Imaginäres in Kultur und Gesellschaft, Konstanz: UVK, S. I89-2I7.

Alkemeyer, Thomas/Gebauer, Gunter/Wiedenhöft, Anja (200I): »Straßenspiele«, in: Jürgen Funke-Wieneke/Klaus Moegling (Hg.): Stadt und Bewegung. Knut Dietrich zur Emeritierung gewidmet. Immenhausen: Prolog, S. 45-67.

Alkemeyer, Thomas/Gebauer, Gunter (2002): »Tiefenstrukturen als Vermittlungen zwischen Spielen und Alltagswelt«, in: Paragrana. Internationale Zeitschrift für Historische Anthropologie II, Heft I, S. $5^{\mathrm{I}-65}$.

Alkemeyer, Thomas/Schmidt, Robert (2003): »Habitus und Selbst. Zur Irritation der körperlichen Hexis in der populären Kultur«, in: Thomas Alkemeyer/Bernhard Boschert/Robert Schmidt/Gunter Gebauer (Hg.): Aufs Spiel gesetzte Körper. Aufführungen des Sozialen in Sport und populärer Kultur. Konstanz: UVK, S. 77-IO2. 
Arendt, Hannah (1998): Vita activa oder Vom tätigen Leben. München: Piper.

Bale, John (I997): »Der Sportplatz: Das Spiel der gezähmten Körper«, in: Zeitschrift für Semiotik I9, Heft I-2, S. 35-48.

Bateson, Gregory (1985): »Eine Theorie des Spiels und der Phantasie«, in: Ökologie des Geistes. Anthropologische, psychologische, biologische und epistemologische Perspektiven. Frankfurt a.M.: Suhrkamp, S. 24I-26I.

Bateson, Gregory (I985a): »Stil, Grazie und Information in der primitiven Kunst«, in: ders.: Ökologie des Geistes. Anthropologische, psychologische, biologische und epistemologische Perspektiven. Frankfurt a.M.: Suhrkamp, S. I82-216.

Bauman, Zygmunt (I992): Moderne und Ambivalenz. Das Ende der Eindeutigkeit. Hamburg: Junius.

Bauman, Zygmunt (2000): Liquid Modernity. Cambridge: Polity Press. Baur, Jürgen/Burrmann, Ulrike (2003): »Jugendliche Sportvereinsmitglieder als >Trittbrettfahrer<?«, in: Sportwissenschaft, H. 4, S. 367-382.

Bech, Henning (I995): »City Sex. Die öffentliche Darstellung der Begierden«, in: Soziale Welt 46, Heft I, S. 5-26.

Beck, Stefan (1997): Umgang mit Technik. Kulturelle Praxen und kulturwissenschaftliche Forschungskonzepte. Berlin: Akademie Verlag.

Benjamin, Walter (I991): »Der Flaneur«, in: ders.: Abhandlungen. Gesammelte Schriften Band I-2. Herausgegeben von Rolf Tiedemann und Hermann Schweppenhäuser. Frankfurt a.M.: Suhrkamp, S. 537-569.

Bette, Karl-Heinrich (1989): Körperspuren. Zur Paradoxie und Semantik moderner Körperlichkeit. Berlin/New York: de Gruyter.

Bette, Karl-Heinrich (I999): »Asphaltkultur. Zur Versportlichung und Festivalisierung urbaner Räume«, in: Karl-Heinrich Bette (Hg.): Systemtheorie und Sport. Frankfurt a.M.: Suhrkamp, S. I92-220.

Bollnow, Otto Friedrich (Hg.) (I994): Mensch und Raum. Stuttgart: Kohlhammer.

Boltanski, Luc (I990): Die Führungskräfte. Die Entstehung einer sozialen Gruppe, Frankfurt a.M./New York: Campus.

Bonß, Wolfgang (I995): Vom Risiko. Unsicherheit und Ungewißheit in der Moderne. Hamburg: Hamburger Edition. 
Boschert, Bernhard (200I): Der Entwurf einer humanen Gesellschaft zwischen Verheißung und Elend. Stationen einer sich selbst problematisierenden Aufklärung in der Literatur der Moderne. Dissertation, Freie Universität Berlin.

Boschert, Bernhard (2002): »Der Sport und der Raum - der Raum des Sports«, in: SportZeiten 2, Heft 2, S. I9-37.

Bourdieu, Pierre (I979a): Entwurf einer Theorie der Praxis auf der ethnologischen Grundlage der kabylischen Gesellschaft. Frankfurt a.M.: Suhrkamp.

Bourdieu, Pierre (I982): Die feinen Unterschiede. Kritik der gesellschaftlichen Urteilskraft. Frankfurt a.M.: Suhrkamp.

Bourdieu, Pierre (I985): Sozialer Raum und >Klassen<. Leçon sur la leçon. Zwei Vorlesungen. Frankfurt a.M.: Suhrkamp.

Bourdieu, Pierre (I986): »Historische Voraussetzungen modernen Sports«, in: Gunter Gebauer/Gerd Hortleder (Hg.): Sport - Eros Tod. Frankfurt a.M.: Suhrkamp, S. 9I-II2.

Bourdieu, Pierre (I992): »Programm für eine Soziologie des Sports«, in: ders.: Rede und Antwort. Frankfurt a.M.: Suhrkamp, S. I93-207.

Bourdieu, Pierre (I993): »Historische und soziale Voraussetzungen des modernen Sports«, in: ders.: Soziologische Fragen. Frankfurt a.M.: Suhrkamp, S. I65-186.

Bourdieu, Pierre (I997): »Der Tote packt den Lebenden«, in: ders.: Der Tote packt den Lebenden. Schriften zu Politik \& Kultur 2. Hamburg: VSA, S. I8-58.

Bourdieu, Pierre (I997a): »Verstehen«, in: ders. et al.: Das Elend der Welt. Zeugnisse und Diagnosen alltäglichen Leidens an der Gesellschaft. Konstanz: UVK, S. 779-822.

Bourdieu, Pierre (200I): Meditationen. Zur Kritik der scholastischen Vernunft. Frankfurt a.M.: Suhrkamp.

Brecht, Bertolt (I967): »Neue Techniken der Schauspielkunst. Über den Gestus (I949-I955)«, in: Ders: Gesammelte Werke, Bd. I6. Frankfurt a.M.: Suhrkamp, S. 753-754.

Bröskamp, Bernd (I998): »Global Player. Sport im Zeitalter der Globalisierung«, in: blätter des informationszentrums 3. Welt, Heft Mai/ Juni, S. 2I-24. 
Cachay, Klaus/Thiel, Ansgar (2000): Soziologie des Sports. Zur Ausdifferenzierung und Entwicklungsdynamik des Sports der modernen Gesellschaft. Grundlagentexte Soziologie. Weinheim/München: Juventa.

Caillois, Roger (1982): Die Spiele und die Menschen. Maske und Rausch. Aus dem Französischen von Sigrid von Massenbach. Frankfurt a.M./Berlin/Wien: Ullstein.

Clarke, John (1976): »Style«, in: Stuart Hall/Tony Jefferson (Eds.): Resistance through Rituals. Youth subcultures in post-war Britain. London: Routledge, S. I75-I9I.

Dangschat, Jens S. (I996): »Raum als Dimension sozialer Ungleichheit und Ort als Bühne der Lebensstilisierung? - Zum Raumbezug sozialer Ungleichheit und von Lebensstilen«, in: Otto G. Schwenk (Hg.): Lebensstil zwischen Sozialstrukturanalyse und Kulturwissenschaft. Opladen: Leske + Budrich, S. 99-I35.

Durkheim, Emil (I9I2): Die elementaren Formen des religiösen Lebens. (Ausgabe I998). Frankfurt a.M.: Suhrkamp.

Eder, Klaus (I989): »Jenseits der nivellierten Mittelstandsgesellschaft. Das Kleinbürgertum als Schlüssel einer Klassenanalyse in fortgeschrittenen Industriegesellschaften«, in: ders. (Hg.): Klassenlage, Lebensstil und kulturelle Praxis. Theoretische und empirische Beiträge zur Auseinandersetzung mit Pierre Bourdieus Klassentheorie. Frankfurt a.M.: Suhrkamp, S. 34I-392.

Eichberg, Henning (I973): Der Weg des Sports in die industrielle Zivilisation. Baden-Baden: Nomos.

Eichberg, Henning (1984): Die historische Relativität der Sachen. Auf dem Weg zu einer kritischen Technikgeschichte. Münster: Lit.

Eichberg, Henning (I986): »Disziplinierungsanstalten und grüne Wellen«, in: Wolfgang Dressen (Hg.): Selbstbeherrschte Körper. Berliner Topografien, Nr. 6. Berlin: Ästhetik \& Kommunikation, S. 27-47.

Eisenberg, Christiane (I999): > English Sports < und deutsche Bürger. Eine Gesellschaftsgeschichte 1800-1939. Paderborn: Schöningh.

Elias, Norbert (1983): »Der Fußballsport im Prozess der Zivilisation«, in: Rolf Lindner (Hg.): Der Satz »Der Ball ist rund « hat eine gewisse philosophische Tiefe. Sport, Kultur, Zivilisation. Berlin: Transit, S. I2-2I.

Elias, Norbert (I994): Die Gesellschaft der Individuen. Herausgegeben von Michael Schröter. Frankfurt a.M.: Suhrkamp. 
Elias, Norbert/Dunning, Eric (2003): Sport und Spannung im Prozess der Zivilisation. Gesammelte Schriften, Bd. 7. Übersetzt von Detlef Bremcke, Wilhelm Hopf und Reinhardt Peter Nippert. Frankfurt a.M.: Suhrkamp.

Featherstone, Mike/Burrows, Roger (I995): »Cultures of technological Embodiment: An Introduction«, in: dies. (Hg.): Cyberspace, Cyberbodies, Cyberpunk. Cultures of technological Embodiment. London/ Thousand Oaks/New Dehli: Sage, S. I-I9.

Fischer-Lichte, Erika (Hg.) (200I): Antrag auf Verlängerung und Finanzierung des Sonderforschungsbereichs 447 Kulturen des Performativen. »Performative Turns« im Mittelalter, in der Frühen Neuzeit und in der Moderne für die Jahre 2002-2003-2004. Berlin.

Fischer-Lichte, Erika/Roselt, Jens (200I): »Attraktion des Augenblicks - Aufführung, Performance, performativ und Performativität«, in: Paragrana. Internationale Zeitschrift für Historische Anthropologie Io, Heft I, S.237-253.

Flick, Uwe (2002): Qualitative Sozialforschung - Eine Einführung. Reinbek: Rowohlt.

Foucault, Michel (I976): Mikrophysik der Macht. Berlin: Merve.

Foucault, Michel (I977): Überwachen und Strafen. Die Geburt des Gefängnisses. Frankfurt a.M.: Suhrkamp.

Foucault, Michel (1978): Dispositive der Macht. Über Sexualität, Wissen und Wahrheit. Berlin: Merve.

Foucault, Michel (I994): »Interview de Michel Foucault«, in: ders.: Dits et écrits. Bd. 4, herausgegeben von Daniel Defert/Francois Ewald. Paris: Gallimard, S. 688-696.

Foucault, Michel (I998): »Andere Räume«, in: Karlheinz Barck/u.a. (Hg.): Aisthesis. Wahrnehmung heute oder Perspektiven einer anderen Ästhetik. Essais. Leipzig: Reclam, S. 34-46.

Franke, Berthold (I990): Die Kleinbürger. Begriff, Ideologie, Politik, Frankfurt a.M./New York: Campus.

Franke, Elk (I985): »Der Raum sportlicher Handlungen - ein übersehenes Thema sportwissenschaftlicher Grundlagendiskussion«, in: Michael Klein (Hg.): Sport, Umwelt und sozialer Raum. Referate der Arbeitstagung der DVS-Sektion >Sportsoziologie in GrevenbroichLangwaden vom 7.10-9.10.1983. Clausthal-Zellerfeld: Czwalina, S. I9-50. 
Gebauer, Gunter (I999): »Bewegte Gemeinden. Über religiöse Gemeinschaften im Sport«, in: Merkur, Sonderheft 9/ıO, S. 936-952.

Gebauer, Gunter (200I): »Der Held und sein Handy. Sport als Habitus und Erzählung«, in: Merkur, H. 62I, S. I-I4.

Gebauer, Gunter (2002): Sport in der Gesellschaft des Spektakels. St. Augustin: Academia.

Gebauer, Gunter (2003): »Von Übermenschen und ihren Kräften«, in: überMenschen. Tagungsband des Kolloquiums »überMenschen. Zur Zukunft des Humanen« des Kollegs Friedrich Nietzsche der Stiftung Weimarer Klassik v. 6.- 8.I2.2002. Weimar: Autoren und Künstler 2003, S. I62-I7I.

Gebauer, Gunter/König, Eugen (I986): »Wohnen, Alltagskultur und Sportengagement im Berliner Stadtteil Siemensstadt«, in: Gunter A. Pilz (Hg.): Sport und Verein, Reinbek: Rowohlt, S.90-I03.

Gebauer, Gunter/Wulf, Christoph (I998): Spiel, Ritual, Geste. Mimetisches Handeln in der sozialen Welt. Reinbek bei Hamburg: Rowohlt. Gebhardt, Winfried/Hitzler, Ronald/Pfadenhauer, Michaela (Hg.) (2000): Events. Soziologie des Außergewöhnlichen. Opladen: Leske + Budrich.

Gehlen, Arnold (I993): Anthropologische und sozialpsychologische Untersuchungen. Reinbek: Rowohlt.

Göhler, Josef/Spieth, Rudolf (I998): »Geschichte der Turngeräte«., in: http://gymnamedia.com/ghent2001/appa/pommel/history_ph_d.htm (Zugriff am 04.03.2004).

Goffman, Erving (I973): Interaktion: Spaß am Spiel. Rollendistanz. München: Piper.

Goffman, Erving (1996): Rahmen - Analyse. Ein Versuch über die Organisation von Alltagserfahrungen. Übersetzt von Hermann Vetter. Frankfurt a.M.: Suhrkamp.

Gumbrecht, Hans Ulrich (I998): »Die Schönheit des Mannschaftssports: American Football - im Stadion und im Fernsehen«, in: Gianni Vattimo/Wolfgang Welsch (Hg.): Medien-Welten Wirklichkeiten. München: Fink, S. 20I-228.

Habermas, Jürgen (I990): Strukturwandel der Öffentlichkeit. Untersuchungen zu einer Kategorie der bürgerlichen Gesellschaft. Frankfurt a.M.: Suhrkamp. 
Habermas, Tilmann (1999): Geliebte Objekte: Symbole und Instrumente der Identitätsbildung. Frankfurt a.M.: Suhrkamp.

Häußermann, Hartmut/Siebel, Walter (I993): »Die Politik der Festivalisierung und die Festivalisierung der Politik. Große Ereignisse in der Stadtpolitik«, in: dies. (Hg.): Festivalisierung der Stadtpolitik. Stadtentwicklung durch große Projekte. Leviathan. Zeitschrift für Sozialwissenschaft, Sonderheft I3, S. 7-3I.

Hahn, Alois (I986): »Soziologische Relevanzen des Stilbegriffs«, in: Hans Ulrich Gumbrecht/Karl-Ludwig Pfeiffer (Hg.): Stil. Geschichten und Funktionen eines kulturwissenschaftlichen Diskurselements. Frankfurt a.M.: Suhrkamp, S. 603-6iI.

Harms, Gerd/Preissing, Christa/Richtermeier, Adolf (I985): »Theoretische Vorüberlegungen. Raumaneignung als zentrale Form der Tätigkeit von Kindern und Jugendlichen in der Stadt«, in: dies. (Hg.): Kinder und Jugendliche in der Großstadt. Berlin: FIPP, S. 932.

Heinemann, Klaus (I998): Einführung in die Soziologie des Sports; 4. Auflage. Schorndorf: Hofmann.

Heinemann, Klaus (200I): »Bausteine einer sozio-ökonomischen Theorie der Sporttechnologie«, in: Albrecht Hummel/Alfred Rütten: Handbuch Technik und Sport. Sportgeräte - Sportausrüstungen Sportanlagen. Beiträge zur Lehre und Forschung im Sport, Bd. I30. Schorndorf: Hofmann, S. II-48.

Heitmeyer, Wilhelm (Hg.) (I997): Was treibt die Gesellschaft auseinander? Frankfurt a.M.: Suhrkamp.

Heitmeyer, Wilhelm/Anhut, Reimund (Hg.) (2000): Bedrohte Stadtgesellschaft. Soziale Desintegrationsprozesse und ethnisch kulturelle Konfliktkonstellationen. Weinheim/München: Juventa.

Hitzler, Roland (I998): »Posttraditionale Vergemeinschaftung. Über neue Formen der Sozialbindung «, in: Berliner Debatte INITIAL 9, Heft I, S. 8I-89.

Holzkamp, Klaus (I995): Lernen. Subjektwissenschaftiche Grundlegung. Frankfurt a.M./New York: Campus.

Kaschuba, Wolfgang (2003): »Repräsentation im öffentlichen Raum«, in: Wolkenkuckucksheim 8, Heft I, S. I-II.

Kemper, Peter/Sonnenschein, Ulrich (Hg.) (200I): Die Kick-Kultur. Zur Konjunktur der Süchte. Leipzig: Reclam. 
Keupp, Heiner (I997): »Die Suche nach Gemeinschaft zwischen Stammesdenken und kommunitärer Individualität«, in: Wilhelm Heitmeyer (Hg.): Was hält die Gesellschaft zusammen? Frankfurt a.M.: Suhrkamp, S. 279-3I2.

Klaus, Elisabeth/Pater, Monika/Schmidt, Uta C. (I997): »Das Gendering neuer Technologien«, in: Das Argument 223, S. 803-818.

König, Eugen (1989): Körper - Wissen - Macht. Studien zur Historischen Anthropologie des Körpers. Berlin: Reimer.

Krais, Beate (I990): »Nachwort zur deutschen Ausgabe«, in: Luc Boltanski: Die Führungskräfte. Die Entstehung einer sozialen Gruppe, Frankfurt a.M./New York: Campus, S. 335-344.

Krais, Beate (200I): Die französische Perspektive auf Social Capital und Implikationen für die gesellschaftspolitische Praxis. Beitrag zum internationalen Workshop der Enquête-Kommission »Zukunft des bürgerschaftlichen Engagements« des I4. Deutschen Bundestages im Plenarbereich Reichstagsgebäude am 25. Juni 200I. Unveröffentlichtes Manuskript. Berlin.

Krais, Beate/Gebauer, Gunter (2002): Habitus. Bielefeld: transcript.

Lamprecht, Markus/Stamm, Hanspeter (I994): Die soziale Ordnung der Freizeit. Soziale Unterschiede im Freizeitverhalten der Schweizer Wohnbevölkerung. Zürich: Seismo.

Lamprecht, Markus/Stamm, Hanspeter (I998): »Das Entwicklungsmuster von Trendsportarten - Zwischen Innovation und Integration«, in: Sporterziehung in der Schule, Heft 4, S. 6-9.

Levin, Thomas Y. (I997): »Geopolitik des Winterschlafs: Zum Urbanismus der Situationisten«, in: Wolkenkuckucksheim 2, Heft 2, S. I-2O.

Löw, Martina (200I): Raumsoziologie. Frankfurt a.M.: Suhrkamp.

Loret, Alain (I996): Génération glisse: dans l'eau, l'air, la neige ... La révolution du sport des »années fun«. Paris: Autrement.

Luhmann, Niklas (1987): Soziale Systeme. Grundriss einer allgemeinen Theorie. Frankfurt a.M.: Suhrkamp.

Luhmann, Niklas (ı997): Die Gesellschaft der Gesellschaft. 2 Teilbände. Frankfurt a.M.: Suhrkamp.

Maresch, Rudolf/Werber, Niels (Hg.) (2002): Raum - Wissen - Macht. Frankfurt a.M.: Suhrkamp. 
Marlovits, Andreas M. (200I): »Snowboarding - Zur Psychologie einer Sportart und heraldischen Funktion einer Gerätschaft«, in: Sportwissenschaft 31, Heft 4, S. 425-436.

Merleau-Ponty, Maurice (I966): Phänomenologie der Wahrnehmung. Berlin: de Gruyter.

Meschnig, Alexander/Stuhr, Matthias (Hg.) (2003): Arbeit als Lebensstil. Frankfurt a.M.: Suhrkamp.

Messing, Manfred/Lames, Martin (I996): Zur Sozialfigur des Sportzuschauers. Niederhausen: Schors.

Meuser, Michael (2002): »Körper und Sozialität. Zur handlungstheoretischen Fundierung einer Soziologie des Körpers«, in: Kornelia Hahn/Michael Meuser (Hg.) (2002): Körperrepräsentationen. Die Ordnung des Sozialen und der Körper. Konstanz: UVK, S. I9-44.

Muchow, Martha/Muchow, Hans (I935): Der Lebensraum des Großstadtkindes. Hamburg: Rowohlt.

Nakane, Chie (I972): Japanese Society. Berkeley: University of California Press.

Nerlich, Michael (I997): Abenteuer oder das verlorene Selbstverständnis der Moderne, München: Gerling Akademie Verlag.

Nickel, Ulrich (I999): »Neue Geräte in der SportPraxis (2). Gesellschaftliche Veränderungen fordern neue Sportgeräte«, in: SportPraxis 6. Wiesbaden: Limpert, S. 4I-42.

Nissen, Ursula (1998): Kindheit, Geschlecht und Raum. Sozialisationstheoretische Zusammenhänge geschlechtsspezifischer Raumaneignung. Weinheim/München: Juventa.

Noller, Peter (2000): »Globalisierung, Raum und Gesellschaft: Elemente einer modernen Soziologie des Raumes«, in: Berliner Journal für Soziologie Io, Heftı, S. 2I-48.

Norden, Gilbert (I998): »Schuhe zum >Eislaufen ohne Eis« und »spiegelglatte, >kaum von einem Eisspiegel zu unterscheidende< Laufflächen«. Eine Geschichte des Rollschuhes und der Rollschuhbahnen bis 1924 unter besondere Berücksichtigung Österreichs, in: Blätter für Technikgeschichte 60, S. II-42.

Plessner, Helmuth (I98I): »Die Stufen des Organischen und der Mensch«, in: ders.: Gesammelte Schriften IV (Herausgegeben von Günter Dux/Udo Marquardt/Elisabeth Ströker), Frankfurt a.M.: Suhrkamp. 
Poser, Stefan (200I): »Der >ultimative Kick<. Oder: Was wäre die Erlebnisgesellschaft ohne ihre technischen Grundlagen?«, in: Kultur Q Technik 3, S. 2I-25.

Pries, Ludger (I996): »Transnationale Soziale Räume. Theoretischempirische Skizze am Beispiel der Arbeitswanderungen Mexico USA«, in: Zeitschrift für Soziologie 25, Heft 6, S. 456-472.

Reichertz, Jo (I992): »Beschreiben oder Zeigen - Über das Verfassen ethnographischer Berichte«, in: Soziale Welt 43, Heft 3, S. 33I-350.

Richter, Norbert (200I): Spiel und Agon vor dem Hintergrund der Anthropologie Helmuth Plessners. Unveröffentlichtes Manuskript. Berlin.

Rittner, Volker (I983): »Zur Soziologie körperbetonter sozialer Systeme«, in: Kölner Zeitschrift für Soziologie und Sozialpsychologie, Sonderheft 25. S. 233-255.

Schivelbusch, Wolfgang (2002): Geschichte der Eisenbahnreise. Zur Industrialisierung von Raum und Zeit im 19. Jahrhundert; 2. Aufl. Frankfurt a.M.: Fischer.

Schmidt, Robert (2002): Pop - Sport - Kultur. Praxisformen körperlicher Aufführungen. Konstanz: UVK.

Schönberger, Klaus/Springer, Stefanie (2003): Subjektivierte Arbeit. Mensch, Organisation und Technik in einer entgrenzten Arbeitswelt. Frankfurt a.M./New York: Campus.

Schulze, Gerhard (I993): Die Erlebnisgesellschaft. Kultursoziologie der Gegenwart. Frankfurt a. Main/New York: Campus.

Schulze, Gerhard (I994): »Milieu und Raum«, in: Peter Noller/Walter Prigge/Klaus Ronneberger (Hg.): Stadt - Welt. Über Globalisierung städtischer Milieus. Franfurt a.M./New York: Campus, S. 4I-53.

Schwabe, Vanessa (2003): BMX-Flatland und das Zueinanderkommen von Körper und Technik. Eine Sportkultur als Indikator für einen neuen Körper-Technik-Trend in der Gesellschaft. Staatsexamensarbeit, Freie Universität Berlin.

Simmel, Georg (I980): »Soziologie der Mahlzeit« (I910), in: ders.: Soziologische Ästhetik. Herausgegeben und eingeleitet von Kurt Lichtblau (I980). Bodenheim: Philo, S. I83-190.

Simmel, Georg (1992): »Der Raum und die räumlichen Ordnungen der Gesellschaft«, in: ders. (Hg.): Soziologie. Untersuchungen über die Formen der Vergesellschaftung. Gesamtausgabe, Bd. II. Heraus- 
gegeben von Otthein Rammstedt. Frankfurt a.M.: Suhrkamp, S. $687-790$.

Simmel, Georg (I993): »Das Problem des Stiles«, in: ders.: Gesamtausgabe, Bd. 8, Aufsätze und Abhandlungen I90I-I908, Bd. II. Frankfurt a.M.: Suhrkamp, S. 382.

Simmel, Georg (I995): »Philosophie der Mode«, in: ders., Gesamtausgabe, Bd. Io. Frankfurt a.M.: Suhrkamp, S. 7-37.

Simmel, Georg (I998): »Die Großstädte und das Geistesleben«, (I903), in: ders.: Soziologische Ästhetik. Herausgegeben und eingeleitet von Kurt Lichtblau. Bodenheim: Philo, S. II9-I33.

Singer, Milton B. (Hg.) (I959): Traditional India. Structure and Change. (Bibliographical Series X). Philadelphia: The American Folklore Society.

Sinus Sociovision (2002): Typologie der Wünsche. Die Sinus-Milieus in Deutschland. Offenburg: Burda Advertising Center GmbH.

Soeffner, Hans-Georg (I986): »Stil und Stilisierung. Punk oder die Überhöhung des Alltags«, in: Hans Ulrich Gumbrecht/Karl-Ludwig Pfeiffer (Hg.): Stil. Geschichte und Funktionen eines kulturwissenschaftlichen Diskurselements. Frankfurt a.M.: Suhrkamp.

Soeffner, Hans-Georg (I995): Die Ordnung der Rituale. Die Auslegung des Alltags 2; 2. Auflage. Frankfurt a.M.: Suhrkamp.

Soeffner, Hans-Georg (2000): Gesellschaft ohne Baldachin, Über die Labilität von Ordnungskonstruktionen. Weilerswist: Velbrück Wissenschaft.

Soeffner, Hans-Georg (200I): »Stile des Lebens. Ästhetische Gegenentwürfe zur Alltagspragmatik«, in: Jörg Huber (Hg): Kultur-Analysen. Interventionen 10. Institut für Theorie der Gestaltung und Kunst Zürich. Wien/New York: Springer, S. 79-II4.

Stern, Martin (2003): »Heldenfiguren im Wagnissport. Zur medialen Inszenierung wagnissportlicher Erlebnisräume«, in: Thomas Alkemeyer/Bernhard Boschert/Robert Schmidt/Gunter Gebauer (Hg.): Aufs Spiel gesetzte Körper. Aufführungen des Sozialen in Sport und populärer Kultur. Konstanz: UVK, S. 37-54.

Stichweh, Rudolf (2000): Die Weltgesellschaft. Soziologische Analysen. Frankfurt a.M.: Suhrkamp. 
Tönnies, Ferdinand (1979): Gemeinschaft und Gesellschaft. Grundbegriffe der reinen Soziologie. Darmstadt: Wissenschaftliche Buchgesellschaft.

Trabant, Jürgen (200I): Über Ruhm, Coolness, Wahrheit und andere Fragen der europäischen Sprachkultur. Wissenschaftlicher Festvortrag. Berlin-Brandenburgische Akademie der Wissenschaften, Jahrbuch 200I. Berlin: Akademie Verlag, S. I68-i95.

Trillitzsch, Manuela (200I): »Vom Laufrad zur Radsporttechnologie«, in: Albrecht Hummel/Alfred Rütten: Handbuch Technik und Sport. Sportgeräte - Sportausrüstungen - Sportanlagen. Beiträge zur Lehre und Forschung im Sport, Bd. I30. Schorndorf: Hofmann, S. I99223.

Turner, Victor (1989): »Das Liminale und das Liminoide in Spiel, $>$ Fluß< und Ritual«, in: ders.: Vom Ritual zum Theater. Der Ernst des menschlichen Spiels. Frankfurt a.M./New York: Qumran, S. 28-94.

Vester, Michael/von Oertzen, Peter/Geiling, Heiko/Hermann, Thomas/Müller, Dagmar (200I): Soziale Milieus im gesellschaftlichen Strukturwandel. Zwischen Integration und Ausgrenzung, Frankfurt a.M.: Suhrkamp.

Waldenfels, Bernhard (1999): Sinnesschwellen. Studien zur Phänomenologie des Fremden, Band 3. Frankfurt a.M.: Suhrkamp.

Weber, Max (1980): Wirtschaft und Gesellschaft. 5. Aufl.Tübingen: Mohr.

Weis, Kurt (1995): »Erlebenswelten in der Stadt - symbolträchtige Räume für Erziehung und Eroberung, Spiel und Kampf. Teil 2«, in: erleben und erlernen 3, Heft 3/4, S. II8-I23.

Whyte, William Foote (1996): Die Street Corner Society. Die Sozialstruktur eines Italienerviertels. Nach der 3., durchgesehenen und erweiterten Auflage aus dem Jahre i98I übersetzt von Reinhard Blomert und Joachim Kalka. Berlin/New York: de Gruyter.

Willems, Herbert/Jurga, Martin (Hg.) (1998): Inszenierungsgesellschaft. Ein einführendes Handbuch. Opladen/Wiesbaden: Westdeutscher Verlag.

Winkler, Joachim (1995): »Der Sport als stilistische Möglichkeit in der Symbolisierung von Lebensführung«, in: ders./Kurt Weis (Hg.): Soziologie des Sports. Theorieansätze, Forschungsergebnisse und Forschungsperspektiven. Opladen: Westdeutscher Verlag, S. 26I-280. 
Wittgenstein, Ludwig (I993): »Philosophische Untersuchungen«, in: ders.: Werkausgabe Bd. I. Frankfurt a.M., 9. Auflage: Suhrkamp, S. 225-6I8.

Zeiher, Helga (I983): »Die vielen Räume der Kinder. Zum Wandel räumlicher Lebensbedingungen seit I945", in: Ulf Preuss-Lausitz u.a. (Hg.): Kriegskinder, Konsumkinder, Krisenkinder. Zur Sozialisationsgeschichte seit dem Zweiten Weltkrieg. Weinheim: Beltz, S. I76I95.

Zeiher, Hartmut/Zeiher, Helga (I994): Orte und Zeiten der Kinder. Soziales Leben im Alltag von Großstadtkindern. Weinheim/München: Juventa.

Zinnecker, Jürgen (I979): »Straßensozialisation. Versuch, einen unterschätzten Lernort $\mathrm{zu}$ thematisieren«, in: Zeitschrift für Pädagogik 5, S. 727-746.

Zinnecker, Jürgen (I990): »Vom Straßenkind zum verhäuslichten Kind. Kindheitsgeschichte im Prozess der Zivilisation«, in: Imbke Behnken (Hg.): Stadtgesellschaft und Kindheit im Prozess der Zivilisation. Konfiguration städtischer Lebensweise zu Beginn des 20. Jahrhunderts. Opladen: Leske + Budrich, S. I43-162. 


\section{Die Titel dieser Reihe:}

Klaus E. Müller

\section{Der sechste Sinn}

Ethnologische Studien zu

Phänomenen der

außersinnlichen Wahrnehmung

Mai 2004, 214 Seiten,

kart., $20,80 €$,

ISBN: 3-89942-203-1

Gunter Gebauer, Thomas

Alkemeyer, Bernhard Boschert,

Uwe Flick, Robert Schmidt

\section{Treue zum Stil}

Die aufgeführte Gesellschaft

Mai 2004, 150 Seiten,

kart., $12,80 €$,

ISBN: 3-89942-205-8

Thomas Lemke

\section{Veranlagung und}

Verantwortung

Genetische Diagnostik

zwischen Selbstbestimmung

und Schicksal

Februar 2004, 140 Seiten,

kart., mit Glossar, 14,80 €,

ISBN: 3-89942-202-3

Karl-Heinrich Bette

\section{$\mathrm{X}$-treme}

Zur Soziologie des Abenteuer-

und Risikosports

Februar 2004, 158 Seiten,

kart., $14,80 €$,

ISBN: 3-89942-204-X
Volkhard Krech

\section{Götterdämmerung}

Auf der Suche nach Religion 2003, 112 Seiten,

kart., $12,80 €$,

ISBN: 3-89942-100-O

Volker Heins

Das Andere der Zivilgesellschaft

Zur Archäologie eines Begriffs 2002, 102 Seiten,

kart., $12,80 €$,

ISBN: 3-933127-88-2

Stefan Weber

Medien - Systeme - Netze

Elemente einer Theorie der

Cyber-Netzwerke

2001, 128 Seiten,

kart., $13,80 €$,

ISBN: 3-933127-77-7

Leseproben und weitere Informationen finden Sie unter: www.transcript-verlag.de 